\title{
Profiles of PATRICIA Tries *
}

\author{
Abram Magner \\ Dept. Computer Science \\ Purdue University \\ W. Lafayette, IN 47907 U.S.A. \\ Email: anmagner@purdue.edu
}

\author{
Wojciech Szpankowski ${ }^{\dagger}$ \\ Dept. Computer Science \\ Purdue University \\ W. Lafayette, IN 47907 U.S.A. \\ Email: spa@cs.purdue.edu
}

\begin{abstract}
A PATRICIA trie is a trie in which non-branching paths are compressed. The external profile $B_{n, k}$, defined to be the number of leaves at level $k$ of a PATRICIA trie on $n$ nodes, is an important "summarizing" parameter, in terms of which several other parameters of interest can be formulated. Here we derive precise asymptotics for the expected value and variance of $B_{n, k}$, as well as a central limit theorem with error bound on the characteristic function, for PATRICIA tries on $n$ infinite binary strings generated by a memoryless source with bias $p>1 / 2$ for $k \sim \alpha \log n$ with $\alpha \in(1 / \log (1 / q)+\epsilon, 1 / \log (1 / p)-\epsilon)$ for any fixed $\epsilon>0$. In this range, $\mathbb{E}\left[B_{n, k}\right]=\Theta\left(\operatorname{Var}\left[B_{n, k}\right]\right)$, and both are of the form $\Theta\left(n^{\beta(\alpha)} / \sqrt{\log n}\right)$, where the $\Theta$ hides bounded, periodic functions in $\log n$ whose Fourier series we explicitly determine. The compression property leads to extra terms in the Poisson functional equations for the profile which are not seen in tries or digital search trees, resulting in Mellin transforms which are only implicitly given in terms of the moments of $B_{m, j}$ for various $m$ and $j$. Thus, the proofs require information about the profile outside the main range of interest. Our derivations rely on analytic techniques, including Mellin transforms, analytic de-Poissonization, the saddle point method, and careful bounding of complex functions.
\end{abstract}

Key Words: Digital trees, PATRICIA trie, tree profiles, analytic combinatorics, analysis of algorithms, recurrences, generating functions, poissonization, Mellin transform, saddle point method.

\section{Introduction}

A digital tree is a fundamental data structure on words in which the storage and retrieval of a word is based on its digits. Digital trees enjoy many important applications, including data compression and distributed hashing $[16,22]$. There are several variations of digital trees, two of the most important being tries and digital search trees. Various parameters of random digital trees have been defined and studied extensively, including height, size, and fill-up level $[20,2,18,13,22]$. Many of these can be rephrased in terms of external and internal profiles. The external profile of a digital tree on $n$ strings at level $k$, denoted by $B_{n, k}$, is the number of leaves at distance $k$ from the root. Study of profiles is motivated by the fact that distributional information about

${ }^{*}$ This work was supported by NSF Center for Science of Information (CSoI) Grant CCF-0939370, and in addition by NSA Grant 130923, and NSF Grants DMS-0800568, and CCF-0830140, and the MNSW grant DEC2013/09/B/ST6/02258, and NIH Grant 1U01CA198941-01.

${ }^{\dagger}$ Also with the Faculty of Electronics, Telecommunications and Informatics, Gdańsk University of Technology, Poland. 
them implies information about many other parameters. For instance, the height $H_{n}$ of a digital tree is the maximum level $k$ such that $B_{n, k}>0$, and studying the distribution of $B_{n, k}$ can give precise asymptotics for the typical height. Moreover, the typical depth $D_{n}$, defined to be depth of a randomly chosen leaf, has a distribution whose mass at any given level is exactly determined by the expected value of the external profile at that level. Many other parameters can similiarly be studied in terms of $B_{n, k}$.

This paper makes a large step in the completion of the project of analyzing the profiles of digital trees under a Bernoulli source model; trie profiles were fully treated in [17], and the expected value and variance of digital search tree profiles were studied in $[5,12]$. We are concerned here with a variant of tries called PATRICIA tries, which address an inefficiency in standard tries [13]. In particular, in a standard trie, if many strings share long prefixes, the result is a tree having many non-branching paths, which is a waste of space. In a PATRICIA trie, non-branching paths are compressed; that is, a non-branching path corresponding to symbols $x_{1} \ldots x_{m}$ is replaced by a single node whose parent edge is labeled with the string $x_{1} \ldots x_{m}$. (see Figure 1 for an illustration). In addition to their use as data structures, PATRICIA tries also arise as combinatorial structures

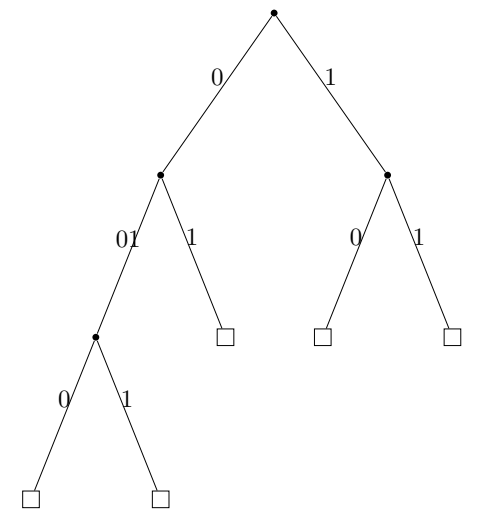

Figure 1: A PATRICIA trie on $n=5$ strings $\left(s_{1}=0010 \ldots, s_{2}=0011 \ldots, s_{3}=01 \ldots, s_{4}=10 \ldots\right.$, $\left.s_{5}=11 \ldots\right)$. Note the path compression involved in the representation of $s_{1}$ and $s_{2}$. The external profile is given by $B_{5,0}=B_{5,1}=0, B_{5,2}=3, B_{5,3}=2$.

which capture the behavior of various processes of interest in computer science and information theory (e.g., in leader election processes without trivial splits [11] and in the solution to Rényi's problem on distinguishing members of a set $[19,2])$.

Here we study the expected value $\mathbb{E}\left[B_{n, k}\right]=\mu_{n, k}$, variance $\operatorname{Var}\left[B_{n, k}\right]=V_{n, k}$, and limiting distributional behavior of the external profile of PATRICIA tries built from $n$ strings generated by a memoryless source with probability of a " 1 " equal to $p>1 / 2$ and probability of a " 0 " equal to $q:=1-p$ (extension of the analysis to any fixed alphabet size is relatively easy). At a very high level, the derivations follow lines well trodden in the analyses of profiles of tries and digital search trees: from the recurrence for the expected value, we derive a functional equation on its Poisson transform, solve this using the Mellin transform, then invert using the saddle point method and analytic de-Poissonization.

The mathematical novelty of the challenges arising in the PATRICIA case, which we solve here, is the fact that, in order to solve the problem for the range of polynomial growth (where $k$ grows logarithmically with $n$ ), we must provide estimates for $\mu_{m, j}$ both to the left and to the right of that range. This comes initially from the peculiar recurrence satisfied by the probability-generating 
function $Q_{n, k}(u)=\mathbb{E}\left[u^{B_{n, k}}\right]$ of the external profile at level $k$ :

$$
Q_{n, k}(u)=\left(p^{n}+q^{n}\right) Q_{n, k}(u)+\sum_{j=1}^{n-1}\left(\begin{array}{l}
n \\
j
\end{array}\right) p^{j} q^{n-j} Q_{j, k-1}(u) Q_{n-j, k-1}(u),
$$

with appropriate initial conditions. The added term $\left(p^{n}+q^{n}\right) Q_{n, k}(u)$ and the incompleteness of the binomial sum are complications that do not arise in the analyses of tries and digital search trees (see [21]). They lead to Mellin transforms that can only be written implicitly in terms of an infinite series involving $\mu_{m, j}$ for $m, j$ outside the range where the profile grows polynomially. Moreover, in the Mellin inversion via the saddle point method, we find that we must handle infinitely many saddle points along the line of integration. Both of these phenomena significantly complicate the inversion of the Mellin transform in the expected value and variance cases and the bounding of the remainder term in the expansion of the characteristic function in the proof of the limiting distribution result.

More precisely, the Poisson transform $\tilde{G}_{k}(z)=\sum_{m \geq 0} \mu_{m, k} \frac{z^{m}}{m !} e^{-m}$ of the expected value sequence $\left(\mu_{n, k}\right)_{n \geq 0}$ satisfies the functional equation

$$
\tilde{G}_{k}(z)=\tilde{G}_{k-1}(p z)+\tilde{G}_{k-1}(q z)+e^{-p z}\left(\tilde{G}_{k}-\tilde{G}_{k-1}\right)(q z)+e^{-q z}\left(\tilde{G}_{k}-\tilde{G}_{k-1}\right)(p z),
$$

and the last two terms present the main challenge, since they do not have closed-form Mellin transforms. We manage to derive a non-explicit formula for the Mellin transform $G_{k}^{*}(s)$ of $\tilde{G}_{k}(z)$ :

$$
G_{k}^{*}(s)=\left(p^{-s}+q^{-s}\right)^{k} \Gamma(s+1) A_{k}(s),
$$

where $A_{k}(s)$ is an infinite series given in terms of $\mu_{m, j}$ :

$$
A_{k}(s)=1+\sum_{j=1}^{k}\left(p^{-s}+q^{-s}\right)^{-j} \sum_{m \geq j}\left(p^{m}+q^{m}\right)\left(\mu_{m, j}-\mu_{m, j-1}\right) \frac{\Gamma(m+s)}{\Gamma(s+1) \Gamma(m+1)} .
$$

Thus, elucidating the analytic properties of $G_{k}^{*}(s)$ requires us to study the asymptotics of $\mu_{m, j}$ in several ranges.

In contrast, the Poisson functional equations for the expected value in tries and digital search trees, respectively, are

$$
\tilde{G}_{k}(z)=\tilde{G}_{k-1}(p z)+\tilde{G}_{k-1}(q z)
$$

(see [17]) and

$$
\tilde{G}_{k}(z)+\tilde{G}_{k}^{\prime}(z)=\tilde{G}_{k-1}(p z)+\tilde{G}_{k-1}(q z)
$$

(see [5]). Both of these result in (more or less) explicitly given Mellin transforms: for tries,

$$
G_{k}^{*}(s)=\left(p^{-s}+q^{-s}\right)^{k} \Gamma(s+1) G_{0}^{*}(s),
$$

and for DSTs,

$$
G_{k}^{*}(s)=\Gamma(s) F_{k}(s),
$$

where $F_{k}(s)$ is a finite linear combination of functions of the form $p^{-\ell_{1} s} q^{-\ell_{2} s}$. Though the Mellin transform in the case of DSTs is still quite complicated, it does not present the same challenges as does the PATRICIA case.

The peculiarities of the recurrence (1) also result in significant challenges in the derivation of the limiting distribution (wherein we appeal to the Lévy continuity theorem): considering the exponential generating function $Q_{k}(u, z)=\sum_{m=0}^{\infty} Q_{m, k}(u) \frac{z^{m}}{m !}$ of $Q_{n, k}(u)$, then taking its logarithm 
$\tilde{l}_{k}(u, z)=\log Q_{k}(u, z)$, we study the asymptotic behavior of the Taylor expansion of $\tilde{l}_{k}(u, z)$ around $u=1$, with $z \rightarrow \infty$. We require precise estimates of the first moments of $B_{n, k}$, and we further need to show that the remainder term is negligible with respect to the first three; to do this, we derive for it an integral representation which again involves quite complicated expressions in terms of $\mu_{m, j}$ for various $m$ and $j$. In this representation of the remainder term, we encounter a function

$$
\frac{Q_{j}(w, p x)-Q_{j-1}(w, p x)+Q_{j}(w, q x)-Q_{j-1}(w, q x)}{Q_{j-1}(w, p x) Q_{j-1}(w, q x)},
$$

and in order to bound this, we find that we need to take into account the extensive cancellation that occurs in the numerator, and we must derive precise lower bounds on $\left|Q_{j-1}(w, c x)\right|$ to handle the denominator. In order to account for the cancellation in the numerator, we again need good bounds for $\mu_{m, j}$ outside the range of polynomial growth.

In the end, we are able to derive precise asymptotic expansions for the expected value and variance of $B_{n, k}$ in the range of polynomial growth: $n \rightarrow \infty$ with $k \sim \alpha \log n$, where, for any fixed $\epsilon>0, \alpha \in(1 / \log (1 / q)+\epsilon, 1 / \log (1 / p)-\epsilon)$ (the left and right endpoints of this interval are associated with the fillup level and height, respectively). Specifically, we show that both the mean and the variance are of the same (explicit) polynomial order of growth (with respect to $n$ ), multiplied by subpolynomial factors and bounded, oscillating functions whose Fourier series we can determine in terms of the function $A_{k}(s)$. The oscillations (which also arise in trie and DST profiles) come from infinitely many regularly spaced saddle points that we observe when inverting the Mellin transform. Moreover, we again find that the Fourier series are phrased in terms of $\mu_{m, j}-\mu_{m, j-1}$, which is a result of the boundary conditions on the recurrences, structurally caused by the compression property of PATRICIA tries. Finally, for the same range, we show that a central limit theorem (with error bound on the characteristic function) holds for the normalized profile $\left(B_{n, k}-\tilde{G}_{k}(n)\right) / \sqrt{\tilde{V}_{k}(n)}$, where $V_{k}(z)$ is the Poisson variance of $B_{n, k}$. Moreover, as a byproduct of our analyses, we get estimates for $\mu_{n, k}$ outside the range of polynomial growth and analytic information about $G_{k}^{*}(s)$ and the Mellin transform $V_{k}^{*}(s)$ of $\tilde{V}_{k}(z)$, all of which will play a key role in the precise analysis of the height and other parameters (which we will tackle in a subsequent paper).

We do not consider in this paper the analysis of the internal profile, because it is a rather trivial extension of that of the external case (one need only consider the residue associated with a simple pole of the relevant Mellin transforms). We also do not handle the symmetric case analysis, for a different reason: since the "saddle point range" which appears in the asymmetric case collapses to the empty set, we expect qualitatively different challenges. We save this discussion for future work. See also [14] for a discussion of the internal profile.

We now discuss the relevant literature about digital trees and their profiles, as well as related parameters. Profiles of tries in both the asymmetric and symmetric cases were studied extensively in [17]. The expected profiles of digital search trees in both cases were analyzed in [5], and the variance for the asymmetric case was treated in [12]. Some aspects of trie and PATRICIA trie profiles (in particular, the concentration of their distributions) were studied using probabilistic methods in $[4,3]$. The analyses in [11] and [9] feature trie recurrences involving incomplete binomial sums with extra terms with similarly complicated Mellin transforms; our recurrence, in contrast with these, is bivariate and features different additional terms, which complicates the analysis. For other parameters of interest, see, e.g., [22, 6]. See also [8] for background on complex asymptotics. In [15], we provided some preliminary analysis of the expected profile in the setting that we consider here.

The plan of the paper is as follows. In Section 2, we introduce some notation, give a precise formulation of the problem, present the main results in detail, and give the high-level ideas behind 
the proofs. In Section 3, we prove the main results.

\section{Main Results}

Here we give some notation that is used in the rest of the paper, present in detail the basic setup, and then give our main theorems and some of the intuition behind their proofs. We then discuss consequences and compare with similar results for other digital tree models. Throughout, the function $T(s)$ is given by

$$
T(s)=p^{-s}+q^{-s} \text {. }
$$

All asymptotic notation is defined with $n \rightarrow \infty$ unless explicitly indicated otherwise.

\section{$2.1 \quad$ Setup}

Throughout this paper, we consider a random PATRICIA trie over $n$ independently generated strings, each an infinite sequence of i.i.d. Bernoulli random variables with probability $p$ of taking the value " 1 " and $q=1-p$ of taking the value " 0 ", with $p>q$. Define $B_{n, k}$ to be the number of external nodes at level $k$ of such a tree.

The fundamental recurrence for $Q_{n, k}(u)=\mathbb{E}\left[u^{B_{n, k}}\right]$, the probability-generating function (PGF) of the external profile, is

$$
Q_{n, k}(u)=\left(p^{n}+q^{n}\right) Q_{n, k}(u)+\sum_{j=1}^{n-1}\left(\begin{array}{l}
n \\
j
\end{array}\right) p^{j} q^{n-j} Q_{j, k-1}(u) Q_{n-j, k-1}(u),
$$

for $n \geq 2$ and $k \geq 1$. This recurrence arises from conditioning on the number $j$ of strings that begin with a "1". If $1 \leq j \leq n-1$ strings start with " 1 ", then $Q_{n, k}(u)$ is a product of contributions from the left and right subtrees. If, on the other hand, all strings start with the same symbol (which happens with probability $p^{n}+q^{n}$ ), then the path compression property applies, and the contribution is $Q_{n, k}(u)$.

The initial and boundary conditions are as follows:

$$
Q_{n, k}(u)= \begin{cases}1 & n=0 \\ u^{\delta[n=1]} & k=0 \\ u^{\delta[k=0]} & n=1 \\ 1 & k \geq n\end{cases}
$$

Now we state the main theorems.

Theorem 1 gives asymptotics for the expected value in the range of polynomial growth (which is contained in the range where $k=\Theta(\log n))$. In particular, it says that the expected external profile grows polynomially with respect to $n$ (with subpolynomial factors), multiplied by a bounded function which is 1-periodic in $\log n$. We note that we can give a somewhat explicit expression (in terms of $\mu_{m, j}$, which can be computed using the recurrence) for the Fourier series of this oscillating function.

We start by deriving a recurrence for $\mu_{n, k}$ : taking a derivative of $Q_{n, k}(u)$ and setting $u=1$, we get

$$
\mu_{n, k}=\left(p^{n}+q^{n}\right) \mu_{n, k}+\sum_{j=1}^{n-1}\left(\begin{array}{c}
n \\
j
\end{array}\right) p^{j} q^{n-j}\left(\mu_{j, k-1}+\mu_{n-j, k-1}\right)
$$


for $n \geq 2$ and $k \geq 1$, with initial/boundary conditions conditions

$$
\mu_{n, k}= \begin{cases}0 & n=0 \\ \delta[n=1] & k=0 \\ \delta[k=0] & n=1 \\ 0 & k \geq n\end{cases}
$$

The high-level steps of the solution of this recurrence (details are given in Section 3.1) are similar to the analysis for other digital trees: we first derive a functional equation for the Poisson transform $\tilde{G}_{k}(z)=\sum_{m \geq 0} \mu_{m, k} \frac{z^{m}}{m !} e^{-z}$ of $\mu_{n, k}$, which gives

$$
\tilde{G}_{k}(z)=\tilde{G}_{k-1}(p z)+\tilde{G}_{k-1}(q z)+e^{-p z}\left(\tilde{G}_{k}-\tilde{G}_{k-1}\right)(q z)+e^{-q z}\left(\tilde{G}_{k}-\tilde{G}_{k-1}\right)(p z),
$$

which we will write as

$$
\tilde{G}_{k}(z)=\tilde{G}_{k-1}(p z)+\tilde{G}_{k-1}(q z)+\tilde{W}_{k, G}(z),
$$

and at this point the goal is to determine asymptotics for $\tilde{G}_{k}(z)$ as $z \rightarrow \infty$ in a cone around the positive real axis; later, de-Poissonization will allow us to directly transfer this asymptotic expansion back to one for $\mu_{n, k}$.

To convert this to an algebraic equation for which we can give a more explicit (though analytically complicated) solution, we use the Mellin transform [7], which, for a function $f: \mathbb{R} \rightarrow \mathbb{R}$ is given by

$$
f^{*}(s)=\int_{0}^{\infty} z^{s-1} f(z) \mathrm{d} z .
$$

Using the Mellin transform identities, we end up with an expression for the Mellin transform $G_{k}^{*}(s)$ of $\tilde{G}_{k}(z)$ of the form

$$
G_{k}^{*}(s)=\Gamma(s+1) A_{k}(s) T(s)^{k},
$$

where $A_{k}(s)$ is an infinite series whose terms involve $\mu_{m, j}-\mu_{m, j-1}$ for various $m$ and $j$, and we recall that $T(s)=p^{-s}+q^{-s}$. Locating and characterizing the singularities of $G_{k}^{*}(s)$ then becomes important. We find that, for any $k, A_{k}(s)$ is entire, with zeros at $s \in \mathbb{Z} \cap[-k,-1]$, so that $G_{k}^{*}(s)$ is meromorphic, with possible simple poles at the negative integers less than $-k$. The fundamental strip of $\tilde{G}_{k}(z)$ then contains $(-k-1, \infty)$.

We then must asymptotically invert the Mellin transform to recover $\tilde{G}_{k}(z)$. The Mellin inversion formula for $G_{k}^{*}(s)$ is given by

$$
\tilde{G}_{k}(z)=\frac{1}{2 \pi i} \int_{\rho-i \infty}^{\rho+i \infty} z^{-s} G_{k}^{*}(s) \mathrm{d} s=\frac{1}{2 \pi i} \int_{\rho-i \infty}^{\rho+i \infty} z^{-s} \Gamma(s+1) A_{k}(s) T(s)^{k} \mathrm{~d} s,
$$

where $\rho$ is any real number inside the fundamental strip associated with $\tilde{G}_{k}(z)$. We evaluate this integral via the saddle point method [22]. Examining $z^{-s} T(s)^{k}$ and solving the associated saddle point equation

$$
\frac{\mathrm{d}}{\mathrm{d} s}[k \log T(s)-s \log z]=0,
$$

we find an explicit formula (6) for $\rho(\alpha)$, the real-valued saddle point of our integrand. The multivaluedness of the logarithm then implies that there are infinitely many regularly spaced saddle points on this vertical line, for which we must account (these lead directly to oscillations in the $\Theta(1)$ factor in the final asymptotics for $\left.\mu_{n, k}\right)$. The main challenge in completing the saddle point analysis is then to elucidate the behavior of $\Gamma(s+1) A_{k}(s)$ for $s \rightarrow \infty$ along vertical lines: it turns 
out that this function inherits the exponential decay of $\Gamma(s+1)$ along vertical lines, and we prove it by splitting the sum defining $A_{k}(s)$ into two pieces, which decay exponentially for different reasons (the first sum decays as a result of the superexponential decay of $\mu_{m, j}$ for $m=\Theta(j)$, which is outside the main range of interest). We end up with an asymptotic expansion for $\tilde{G}_{k}(z)$ as $z \rightarrow \infty$ in terms of $A_{k}(s)$.

Finally, we must analyze the convergence properties of $A_{k}(s)$ as $k \rightarrow \infty$. We find that it converges uniformly on compact sets to a function $A(s)$ (which is, because of the uniformity, entire). We then apply Lebesgue's dominated convergence theorem to conclude that we can replace $A_{k}(s)$ with $A(s)$ in the final asymptotic expansion of $\tilde{G}_{k}(z)$. All of this yields the following theorem (the proof is in Section 3.1).

Theorem 1 (Expected external profile for $k \sim \alpha \log n)$. Let $\epsilon>0$ be independent of $n$ and $k$, and fix $\alpha \in\left(\frac{1}{\log (1 / q)}+\epsilon, \frac{1}{\log (1 / p)}-\epsilon\right)$. Then for $k=k_{\alpha, n} \sim \alpha \log n$,

$$
\mathbb{E}\left[B_{n, k}\right]=H\left(\rho(\alpha), \log _{p / q}\left(p^{k} n\right)\right) \cdot \frac{n^{\beta(\alpha)}}{\sqrt{2 \pi \kappa_{*}(\rho(\alpha)) k}}\left(1+O\left(k^{-1 / 2}\right)\right)
$$

where

$$
\begin{aligned}
\rho(\alpha) & =-\frac{1}{\log (p / q)} \log \left(\frac{\alpha \log (1 / q)-1}{1-\alpha \log (1 / p)}\right), \\
\beta(\alpha) & =\alpha \log (T(\rho(\alpha)))-\rho(\alpha), \\
\kappa_{*}(\rho) & =\frac{p^{-\rho} q^{-\rho}(\log (p / q))^{2}}{T(\rho)^{2}},
\end{aligned}
$$

and $H(\rho, x)$ (see Figure 2) is a non-zero periodic function with period 1 in $x$ given by

$$
H(\rho, x)=\sum_{j \in \mathbb{Z}} A\left(\rho+i t_{j}\right) \Gamma\left(\rho+1+i t_{j}\right) e^{-2 j \pi i x}
$$

where $t_{j}=2 \pi j / \log (p / q)$, and

$$
A(s)=1+\sum_{j=1}^{\infty} T(s)^{-j} \sum_{n=j}^{\infty} T(-n)\left(\mu_{n, j}-\mu_{n, j-1}\right) \frac{\phi_{n}(s)}{n !}
$$

where $\phi_{n}(s)=\prod_{j=1}^{n-1}(s+j)$ for $n>1$ and $\phi_{n}(s)=1$ for $n \leq 1$. Here, A(s) is an entire function which is zero at the negative integers.

Moreover, we have the following superexponentially decaying upper bound on $\mu_{n, k}$ when $k=$ $\Theta(n)$ : for any $C>0$, there exist $c_{1}, c_{2}>0$ such that, for $n$ large enough, whenever $m \geq C n$,

$$
\mu_{n, m} \leq c_{1} n ! e^{-c_{2} m^{2}} \quad m=\Theta(n) .
$$

Moving to the variance $V_{n, k}=\sigma_{n, k}^{2}$ of the external profile, we start with the recurrence for the second factorial moment $c_{n, k}=\mathbb{E}\left[B_{n, k}\left(B_{n, k}-1\right)\right]$, which is easily derived from that for $Q_{n, k}(u)$ :

$$
c_{n, k}=\left(p^{n}+q^{n}\right) c_{n, k}+\sum_{j=1}^{n-1}\left(\begin{array}{c}
n \\
j
\end{array}\right) p^{j} q^{n-j}\left(c_{j, k-1}+c_{n-j, k-1}+2 \mu_{j, k-1} \mu_{n-j, k-1}\right),
$$



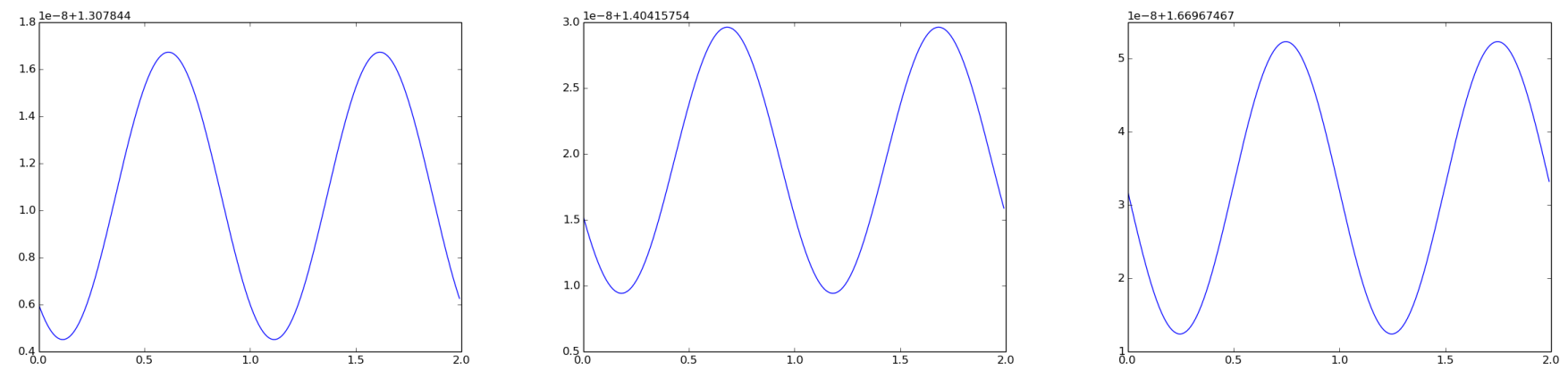

Figure 2: Plots of $H(\rho, x)$ for $\rho=-0.5,0,0.5$.

for $n \geq 2, k \geq 1$, with initial/boundary conditions

$$
c_{n, k}=0 \quad n<2, k<1 \text {, or } k \geq n .
$$

To solve this, we define the Poisson variance $\tilde{V}_{k}(z)$ as

$$
\tilde{V}_{k}(z)=\tilde{C}_{k}(z)+\tilde{G}_{k}(z)-\tilde{G}_{k}(z)^{2},
$$

where $\tilde{C}_{k}(z)=\sum_{m \geq 0} c_{m, k} \frac{z^{m}}{m !} e^{-z}$ is the Poisson transform of $c_{n, k}$. It turns out that $\tilde{V}_{k}(z)$ satisfies a recurrence reminiscent of that of $\tilde{G}_{k}(z)$ :

$$
\tilde{V}_{k}(z)=\tilde{V}_{k-1}(p z)+\tilde{V}_{k-1}(q z)+\tilde{W}_{k, V}(z)
$$

where $\tilde{W}_{k, V}(z)$ is analogous to $\tilde{W}_{k, G}(z)$ appearing in the expected value case (see the proof for its definition).

The subsequent steps (Mellin transform, Mellin inversion via saddle point method, and dePoissonization) are very similar to those in the expected value case. The analytic challenges are essentially the same (except for a detail in the de-Poissonization step, in which we must estimate $n \tilde{G}_{k}^{\prime}(n)^{2}$ ), and we have formulated the lemmas in the analysis of the expected value so that they are readily applicable to the variance case. This results in the following theorem (the proof is given in Section 3.2).

Theorem 2 (Variance). Let $k$ be as in Theorem 1. Then

$$
\operatorname{Var}\left[B_{n, k}\right]=M\left(\rho(\alpha), \log _{p / q}\left(p^{k} n\right)\right) \cdot \frac{n^{\beta(\alpha)}}{\sqrt{2 \pi \kappa_{*}(\rho(\alpha)) k}}\left(1+O\left(k^{-1 / 2}\right)\right)=\Theta\left(\mathbb{E}\left[B_{n, k}\right]\right),
$$

where $\beta(\alpha)$ is as defined in (7), and, for every fixed $\rho, M(\rho, x)$ is a nonzero periodic function with period 1 in $x$, given by the Fourier series

$$
M(\rho, x)=\sum_{j \in \mathbb{Z}} B\left(\rho+i t_{j}\right) \Gamma\left(\rho+1+i t_{j}\right) e^{-2 j \pi i x}
$$

where

$$
B(s)=1-(s+1) 2^{-(s+2)}+\sum_{j=1}^{\infty} T(s)^{-j} \frac{W_{j, V}^{*}(s)}{\Gamma(s+1)}
$$


Here, $W_{j, V}^{*}(s)$ is given by the expression (34). Note that $B(s)$ shares many of the properties of $A(s)$ : it is entire, with zeros at the negative integers.

Moreover, we have the following superexponentially decaying bound on the second factorial moment $c_{n, k}$ (and hence $V_{n, k}$ ) when $k=\Theta(n)$ : for all $C \in(0,1]$, there exist positive constants $C_{1}, C_{2}$ such that, for all $n$ and $k \geq C n$,

$$
c_{n, k} \leq C_{1} n ! e^{-C_{2} k^{2}} \quad k=\Theta(n) .
$$

Finally, we show that the normalized external profile satisfies a central limit theorem. The proof uses the Lévy continuity theorem. Since this entails estimating the characteristic function of $\left(B_{n, k}-\mu_{n, k}\right) / \sigma_{n, k}$ as $n \rightarrow \infty$, we naturally find ourselves studying $\tilde{Q}_{k}(u, z)$, the Poisson transform of the probability-generating function $Q_{n, k}(u)$ of $B_{n, k}$. In fact, we take the logarithm and study $\tilde{l}_{k}(u, z)=\log \left(Q_{k}(u, z)\right)$, which we find, using the Taylor series expansion of $\tilde{l}_{k}(u, z)$ with respect to $u \rightarrow 1$ (we set $u=e^{\tau / \sigma_{n, k}}$, for $\tau=i t, t \in \mathbb{R}$ ), is given by

$$
\tilde{l}_{k}(u, z)=z+\tilde{G}_{k}(z)(u-1)+\frac{\tilde{V}_{k}(z)-\tilde{G}_{k}(z)}{2}(u-1)^{2}+\frac{(u-1)^{3}}{3 !} R\left[\tilde{l}_{k}(u, z),\right.
$$

where $R[\tilde{l}]_{k}(u, z)$ is a remainder term which we must show to be negligible with respect to the other two terms whenever $u \rightarrow 1$ quickly enough.

Everything said so far regarding the proof of the limiting distribution result is fairly standard in the world of digital tree analysis. The new, challenging part of our analysis is showing that the remainder term in the expansion of $\tilde{l}_{k}(u, z)$ is negligible. Using Cauchy's integral formula, we are able to give an exact formula for it:

$$
\begin{aligned}
\frac{1}{3 !} R[\tilde{l}]_{k}(u, z) & =\sum_{j=0}^{k}\left(\begin{array}{c}
k \\
j
\end{array}\right) \frac{1}{2 \pi i} \oint_{\mathcal{C}} \frac{\log \left(1+(w-1) p^{j} q^{k-j} z e^{-p^{j} q^{k-j} z}\right)}{(w-1)^{3}(w-u)} \mathrm{d} w \\
& +\sum_{j=0}^{k} \sum_{m=0}^{k-j}\left(\begin{array}{c}
k-j \\
m
\end{array}\right) \frac{1}{2 \pi i} \oint_{\mathcal{C}} \frac{\tilde{h}_{j}\left(w, p^{m} q^{k-j-m} z\right)}{(w-1)^{3}(w-u)} \mathrm{d} w,
\end{aligned}
$$

where $\tilde{h}_{k}(u, z)$ is a function given in terms of $Q_{k}(u, z)$ and $Q_{k-1}(u, z)$ and $\mathcal{C}$ is a contour enclosing both 1 and $u$. The first sum we bound by writing the $j$ th summand as $e^{\nu(j)}$, for an explicitly determined function $\nu$, then taking derivatives to find the term which contributes maximally. As a result, we find that the contribution of the first sum is $O\left(n^{\beta(\alpha)}\right)$. We bound the second, more complicated summation by proving precise estimates of the asymptotics of $Q_{j}(u, x)$ and $\left(Q_{j}-\right.$ $\left.Q_{j-1}\right)(w, x)$ (which exhibits a significant amount of cancellation) for various ranges of $x$ and $j$. We then split the summations appropriately and apply the asymptotic estimates to conclude that the second sum is also $O\left(n^{\beta(\alpha)}\right)$. This establishes a central limit theorem in the Poisson model, and we finally transfer the result to the Bernoulli model via a "bare-hands" de-Poissonization (i.e., a saddle point evaluation of the Cauchy integral which gives the inverse Poisson transform of $\tilde{G}_{k}(z)$ ) to conclude the following (the proof is given in Section 3.3).

Theorem 3 (Limiting distribution). For $k$ as in Theorem 1 and $\sigma_{n, k}^{2}=V_{n, k}$,

$$
\frac{B_{n, k}-\mu_{n, k}}{\sigma_{n, k}} \stackrel{D}{\rightarrow} \mathcal{N}(0,1) .
$$

More precisely,

$$
\mathbb{E}\left[\exp \left(\tau \frac{B_{n, k}-\tilde{G}_{k}(n)}{\sigma_{n, k}}\right)\right]=\exp \left(\frac{\tau^{2}}{2}\left(1+O\left(V_{n, k}^{-1 / 2}\right)\right)\right),
$$

uniformly for $\tau=i t, t \in \mathbb{R}$. 
We note that, although $\tilde{G}_{k}(n) \sim \mu_{n, k}$ by de-Poissonization, the chosen normalization results in a better relative error than if we had subtracted $\mu_{n, k}$.

Comparing with the limiting distribution derivation for tries, we remark that the main difference lies in the bounding of the error term in (17), where, again, we must work with the non-explicitly known functions $Q_{j}(w, x)$ and their differences. A limiting distribution analysis for DST profiles remains an open problem.

\section{Proofs}

For a sequence of functions $f_{j}: \mathbb{C} \rightarrow \mathbb{C}, j=0, \ldots$, we define $T[f]_{k}: \mathbb{C} \rightarrow \mathbb{C}$, for $k \geq 1$, by

$$
T[f]_{k}(z)=e^{-p z}\left(f_{k}(q z)-f_{k-1}(q z)\right)+e^{-q z}\left(f_{k}(p z)-f_{k-1}(p z)\right) .
$$

We define $L[f]_{k}: \mathbb{C} \rightarrow \mathbb{C}$, for $k \geq 0$, by

$$
L[f]_{k}(z)=f_{k}(p z)+f_{k}(q z) .
$$

Both are trivially seen to be linear (where addition and scalar multiplication of sequences of functions are defined componentwise), which is helpful in the calculations that follow.

For $\theta \in[0, \pi)$ We denote by $\mathcal{C}(\theta)$ the cone around the positive real axis with angle $\theta$; that is,

$$
\mathcal{C}(\theta)=\{z \in \mathbb{C}:|\arg (z)| \leq \theta\}
$$

and for any $R>0$,

$$
\mathcal{C}(\theta, R)=\mathcal{C}(\theta) \cap\{z \in \mathbb{C}|| z \mid \leq R\}
$$

i.e., $\mathcal{C}(\theta, R)$ is the truncated cone of radius $R$.

\subsection{Proof of Theorem 1}

In this section, we prove the estimate (5) of Theorem 1. We relegate the proof of (11) to Appendix 4.2.2.

Our starting point is the Poisson transform $\tilde{G}_{k}(z)=e^{-z} \sum_{n \geq 0} \mu_{n, k} \frac{z^{n}}{n !}$, which satisfies the recurrence

$$
\tilde{G}_{k}(z)=\tilde{G}_{k-1}(p z)+\tilde{G}_{k-1}(q z)+\tilde{W}_{k, G}(z)
$$

where

$$
\tilde{W}_{k, G}(z)=T[\tilde{G}]_{k}(z)
$$

with initial condition $\tilde{G}_{0}(z)=z e^{-z}$. We then apply the Mellin transform [7]

$$
\int_{0}^{\infty} z^{s-1} \tilde{G}_{k}(z) \mathrm{d} z
$$

to $\tilde{G}_{k}(z)$ to get a functional equation for $G_{k}^{*}(s)$ :

$$
G_{k}^{*}(s)=T(s) G_{k-1}^{*}(s)+W_{k, G}^{*}(s)
$$

with $G_{0}^{*}(s)=\Gamma(s+1)$, where the fundamental strip associated with $\tilde{G}_{0}(z)$ is $\Re(s) \in(-1, \infty)$. 
We define $I_{j}=\{s: \Re(s) \in(-j-1, \infty)\}$, so that the fundamental strip of $\tilde{G}_{0}(z)$ becomes $I_{0}$. In fact, we will show that the fundamental strip of each $\tilde{G}_{k}(z)$ contains $I_{k}$. It suffices to analyze the growth of $\tilde{G}_{k}(z)$ as $z \rightarrow 0$ and $z \rightarrow \infty$ on the real axis. For $z \rightarrow 0$,

$$
\tilde{G}_{k}(z)=e^{-z}\left(\mu_{k+1, k} \frac{z^{k+1}}{(k+1) !}+O\left(z^{k+2}\right)\right)=O\left(z^{k+1}\right) .
$$

In order to bound the growth of $\tilde{G}_{k}(z)$ as $z \rightarrow \infty$ for each $k$, we use the following lemma.

Lemma 1 (Growth of $\tilde{G}_{k}(z)$ as $z \rightarrow \infty$, upper bounds). Fix any $\theta \in(0, \pi / 2)$.

(i) For any $\epsilon>0$, there exist some $R \in \mathbb{R}^{+}$and positive $C=C(R)$ such that, for $z \in \mathcal{C}(\theta)$ with $|z|>R$

$$
\left|\tilde{G}_{k}(z)\right| \leq C|z|^{1+\epsilon}
$$

for any $k \geq 0$.

(ii) For any fixed $C>0$, there exists $C^{\prime}>0$ such that, for all $j \leq C, z \in \mathcal{C}(\theta)$, and $\epsilon>0$,

$$
\left|\tilde{G}_{j}(z)\right| \leq C^{\prime}|z|^{1+\epsilon} e^{-q^{C}|z| \cos (\theta)} .
$$

The proof can be found in Appendix 4.2.1. Part (ii) implies that the right endpoint of the fundamental strip of each $\tilde{G}_{k}(z)$ is $\infty$, so that each $\tilde{G}_{k}(z)$ is analytic at least in $I_{k}$, as claimed.

Now, in order to derive a formula for $G_{k}^{*}(s)$, we note that, for any $s \in \bigcap_{j=0}^{\infty} I_{j}=I_{0}$, we can iterate the recurrence (20) to get

$$
G_{k}^{*}(s)=T(s)^{k}\left(G_{0}^{*}(s)+\sum_{j=1}^{k} T(s)^{-j} W_{j, G}^{*}(s)\right) .
$$

It remains to determine $W_{j, G}^{*}(s)$, which we do, for arbitrary $j$, by expressing $e^{-(1-c) z} \tilde{G}_{j}(c z)$ as

$$
\sum_{m \geq j+1} e^{-z} \mu_{m, j} \frac{(c z)^{m}}{m !}
$$

then applying the Mellin transform to each term (the interchange of integrals may be justified by, e.g., Tonelli's theorem). Factoring out $\Gamma(s+1)$ results in the expression

$$
G_{k}^{*}(s)=T(s)^{k} A_{k}(s) \Gamma(s+1),
$$

where

$$
A_{k}(s)=1+\sum_{j=1}^{k} T(s)^{-j} \sum_{n=j}^{\infty} T(-n)\left(\mu_{n, j}-\mu_{n, j-1}\right) \frac{\phi_{n}(s)}{n !},
$$

with $\phi_{n}(s)$ as in the statement of Theorem 1 :

$$
\phi_{n}(s)= \begin{cases}\prod_{j=1}^{n-1}(s+j) & n>1 \\ 1 & n \leq 1 .\end{cases}
$$

Provided that we can show that this expression is analytic for any $s \in I_{k}$, we can then extend this equality to $I_{k}$ by analytic continuation.

In order to do this, we need a lemma to the effect that certain series (which appear in the expression for $A_{k}(s)$ ) converge absolutely and are entire. This lemma will be stated in greater generality than might appear immediately necessary; this is so that we can apply it later in a similar situation in the variance analysis. 
Lemma 2 (Convergence and analyticity of a class of series). Let $F_{j}(s)$ be given by

$$
F_{j}(s)=\sum_{n=j}^{\infty} e^{-g(n)} \frac{\phi_{n}(s)}{n !}
$$

with $g(n)=\Omega(n)$. Then

(i) the sum defining $F_{j}(s)$ converges absolutely for all $s \in \mathbb{C}$, and

(ii) $F_{j}(s)$ is entire.

The proof of this lemma can be found in Appendix 4.1.

We now demonstrate the existence and analyticity of $A_{k}(s)$. Since the outer sum of $A_{k}(s)$ has a finite number of terms for any given $k$, showing existence boils down to showing that the inner sum converges absolutely for all $s \in \mathbb{C}$. For this, we can use Lemma 2, part (i). In the case of $A_{k}(s)$, we note that $T(-n) \leq p^{n}+p^{n}=2 p^{n}$, and $\mu_{n, j}-\mu_{n, j-1} \leq n-0=n$. This yields $T(-n)\left(\mu_{n, j}-\mu_{n, j-1}\right)=e^{-\Theta(n)}$, so that we can take $g(n)=\Theta(n)$, which satisfies the condition required by Lemma 2 . This establishes existence of $A_{k}(s)$.

To show that $A_{k}(s)$ is entire, it suffices to apply Lemma 2, part (ii). Then, since $G_{k}^{*}(s)$ and $T(s)^{k} A_{k}(s)$ are analytic at $s=\{-k,-k+1, \cdots-1\}$ while $\Gamma(s+1)$ is not, and $T(s)^{k} \neq 0, A_{k}(s)=0$ for these points.

We summarize the above derivation in the following theorem.

Theorem 4 (Exact formula, fundamental strip for $G_{k}^{*}(s)$ ). For all $k \geq 1$ and all $s$ for which $G_{k}^{*}(s)$ is holomorphic,

$$
G_{k}^{*}(s)=T(s)^{k} A_{k}(s) \Gamma(s+1),
$$

where $A_{k}(s)$ is an entire function given by

$$
A_{k}(s)=1+\sum_{j=1}^{k} T(s)^{-j} \sum_{n=j}^{\infty} T(-n)\left(\mu_{n, j}-\mu_{n, j-1}\right) \frac{\phi_{n}(s)}{n !},
$$

with $\phi_{n}(s)$ as in the statement of Theorem 1:

$$
\phi_{n}(s)= \begin{cases}\prod_{j=1}^{n-1}(s+j) & n>1 \\ 1 & n \leq 1 .\end{cases}
$$

Furthermore, $A_{k}(s)=0$ for $s=-1, \ldots,-k$, so that $G_{k}^{*}(s)$ is analytically continuable everywhere, except possibly at the negative integers less than $-k$. Thus, the fundamental strip of $G_{k}^{*}(s)$ contains the strip $\Re(s) \in(-k-1, \infty)$.

\subsubsection{Further properties of $A_{k}(s)$}

Here we prove some technical lemmas about $A_{k}(s)$ that will play a role in the inversion of the Mellin transform. For convenience, we write $X_{k}(s)=A_{k}(s) \Gamma(s+1)$. We will prove that $X_{k}(s)$ converges as $k \rightarrow \infty$ to a function $X(s)=A(s) \Gamma(s+1)$ pointwise and uniformly on compact sets (so that $X(s)$ is entire). Then we will show that $X_{k}(s)$ inherits the exponential decay of $\Gamma(s+1)$ along vertical lines. 
Pointwise CONVERGEnce of $X_{k}(s)$ AND Related SERIES

In the next lemma, we prove pointwise convergence of a class of series related to $A_{k}(s)$. Combining this with the upper bound (11) will give us a pointwise convergence result for $A_{k}(s)$. In fact, the convergence will turn out to be uniform for $s$ in any compact set, which implies that $A_{k}(s)$ converges to a function $A(s)$ which is entire.

Lemma 3 (Pointwise convergence of a class of series). Let

$$
U_{k}(s)=\sum_{m=0}^{k} T(s)^{-m} \sum_{n^{\prime}=m}^{\infty} e^{-\Theta\left(n^{\prime}\right)} \eta_{n^{\prime}, m} \frac{\Gamma\left(n^{\prime}+s\right)}{\Gamma\left(n^{\prime}+1\right)},
$$

for any $\eta_{n^{\prime}, m}$ which satisfies

- Superexponential decay for $n^{\prime} \leq C m$ : for any $C>0$, there exists some function $g(m) \geq 0$ satisfying $g(m) \gg m$ such that, if $n^{\prime} \leq C m$ and $m$ is sufficiently large, then

$$
\left|\eta_{n^{\prime}, m}\right| \leq e^{-g(m)}
$$

- Polynomial uniform upper bound: $\left|\eta_{n^{\prime}, m}\right| \leq c_{1} n^{\prime c_{2}}$ for some constants $c_{1}, c_{2}>0$ and all $n^{\prime}, m$.

Let $U(s)=U_{\infty}(s)$. Then $U(s)$ is absolutely convergent for any fixed $s=\rho+i t \in \mathbb{C}$ for which $\left|U_{k}(s)\right|<\infty$ for $k$ large enough.

Proof. Define

$$
b_{m}(s)=T(s)^{-m} \sum_{n^{\prime}=m}^{\infty} e^{-\Theta\left(n^{\prime}\right)} \eta_{n^{\prime}, m} \frac{\Gamma\left(n^{\prime}+s\right)}{\Gamma\left(n^{\prime}+1\right)} .
$$

I.e., $b_{m}(s)$ is the $m$ th term of the series defining $U(s)$. The plan is to show that $\left|b_{m}(s)\right|$ is upper bounded by the tail of a convergent geometric series. Intuitively, for $m=\Theta\left(n^{\prime}\right)$, the terms of the sum are small because $\eta_{n^{\prime}, m}$ is. For larger $n^{\prime}$, the same is true as a result of the smallness of $e^{-\Theta\left(n^{\prime}\right)}$. More precisely, we have the following, for some $c>1$ which we will choose later:

$$
\left|b_{m}(s)\right| \leq|T(s)|^{-m} \sum_{n^{\prime}=m}^{c m} e^{-\Theta\left(n^{\prime}\right)}\left|\eta_{n^{\prime}, m}\right|\left|\frac{\Gamma\left(n^{\prime}+s\right)}{\Gamma\left(n^{\prime}+1\right)}\right|+|T(s)|^{-m} \sum_{n^{\prime}=c m+1}^{\infty} e^{-\Theta\left(n^{\prime}\right)}\left|\eta_{n^{\prime}, m}\right|\left|\frac{\Gamma\left(n^{\prime}+s\right)}{\Gamma\left(n^{\prime}+1\right)}\right| .
$$

Since $s$ is fixed, $\left|\frac{\Gamma\left(n^{\prime}+s\right)}{\Gamma\left(n^{\prime}+1\right)}\right| \sim n^{\prime \rho}$ as $n^{\prime} \rightarrow \infty$ (which is the case when $m \rightarrow \infty[1]$ ).

Upper bounding the first sum: Since $c>1, n^{\prime} \leq c m$ implies $m \geq C n^{\prime}$, with $0<C<1$, so that we can apply the superexponential decay of $\left|\eta_{n^{\prime}, m}\right|$, which yields the following upper bound for the first sum:

$$
|T(s)|^{-m} e^{-\Theta(m)} e^{-g(m)} \Theta\left(m^{\rho+1}\right) .
$$

Since $g(m) \gg m$, provided $m$ is sufficiently large, this can be upper bounded by any $e^{-c_{*} m}$ for any $c_{*}>0$.

Upper bounding the second sum: For the second sum, by the uniform upper bound on $\left|\eta_{n^{\prime}, m}\right|$, we can upper bound

$$
e^{-\Theta\left(n^{\prime}\right)}\left|\eta_{n^{\prime}, m} \frac{\Gamma\left(n^{\prime}+s\right)}{\Gamma\left(n^{\prime}+1\right)}\right|
$$


by $e^{-\Theta\left(n^{\prime}\right)} n^{\prime \Theta(1)}=e^{-\Theta\left(n^{\prime}\right)}$, so that the sum is upper bounded by

$$
e^{-c \Theta(m)}
$$

which gives an upper bound of

$$
e^{-m \log |T(s)|-c \Theta(m)}
$$

for the second term, where the $\Theta(\cdot)$ hides constants depending only on the uniform bound on $\left|\eta_{n^{\prime}, m}\right|$ and $\Re(s)$. Since $|T(s)|$ is fixed, so long as $c$ is sufficiently large (dependent only on $\Re(s)$ ), this is exponentially decaying to 0 . Thus, $\left|b_{m}(s)\right|$ can be bounded by the $m$ th tail of a convergent geometric series, so that it is at least exponentially decaying in $m$, which implies absolute convergence of $|U(s)|$ by the ratio test.

In fact, for any compact domain $\Omega \subset \mathbb{C}$, the convergence is uniform for $s \in \Omega$ (this is a trivial modification of the above proof). We also have the following classical fact about uniform convergence of analytic functions [23]:

Theorem 5 (Uniform convergence of analytic functions). Let $S$ be an open subset of $\mathbb{C}$, and let $\left\{f_{n}\right\}_{n=1}^{\infty}$ be a sequence of functions from $S \rightarrow \mathbb{C}$. If there is a function $f: S \rightarrow \mathbb{C}$ such that, for each compact subset $D \subseteq S,\left\{f_{n}\right\}$ converges uniformly for $s \in D$ to $f$, then $f$ is analytic on $S$.

Applying Theorem 5 and Lemma 3 to $A_{k}(s) \Gamma(s+1)$ (justified by the superexponential decay property of $\mu_{m, j}$ when $j=\Theta(m)$, the upper bound $\mu_{m, j} \leq m$ for all $m$, and the fact that $A_{k}(s) \Gamma(s+$ 1 ) is analytic everywhere except possibly at the integers less than $-k$ ), we have the following corollary.

Corollary 1 (Convergence of $A_{k}(s) \Gamma(s+1)$ ). Recall that $X_{k}(s)=A_{k}(s) \Gamma(s+1)$. Then the sequence $\left\{X_{k}\right\}$ converges pointwise to an entire function $X(s)$, uniformly on any compact set. Moreover, for any $s \in \mathbb{C}$,

$$
\left|X_{k}(s)\right| \leq|\Gamma(s+1)|+\sum_{m=1}^{\infty}|T(s)|^{-m} \sum_{n=m}^{\infty} T(-n)\left|\eta_{n, m}\right|\left|\frac{\Gamma(n+s)}{\Gamma(n+1)}\right| .
$$

DECAY OF $X_{k}(s)$ ALONG VERTICAL LINES

In this section, we analyze the decay of $X_{k}(s)=A_{k}(s) \Gamma(s+1)$ along vertical lines (that is, for $s=\rho+i t$, where $\rho$ is fixed and $|t| \rightarrow \infty)$. This is a key ingredient in the justification of the application of the saddle point method to the inverse Mellin integral.

It turns out that the decay is exponential. We will show this by proving a more general lemma, from which the exponential decay of $X_{k}(s)$ follows as a special case.

Lemma 4 (Decay of $X_{k}(s)$ along vertical lines). Let $U_{k}(s)$ be as in Lemma 3, with an additional condition on $\eta_{n^{\prime}, m}$ : for any $n^{\prime}$ and $m$ with $m \geq n^{\prime}$, we stipulate that

$$
\eta_{n^{\prime}, m}=0 \quad m \geq n^{\prime} .
$$

Then for any $\rho \in \mathbb{R}$, there exist constants $\gamma, r>0$ such that, for $s=\rho+i t$ with $|t|$ sufficiently large, and for any $k$,

$$
\left|U_{k}(s)\right| \leq e^{-\gamma|t|^{r}}
$$

The same holds for $U(s)$ in place of $U_{k}(s)$. 
Proof. For the proof, we first recall a standard fact about the $\Gamma$ function: there exists some positive constant $C$ such that, as $z=\rho+i t \rightarrow \infty$ in a cone $|\arg (z)| \leq \pi-\epsilon$, for any fixed $\epsilon>0$,

$$
|\Gamma(\rho+i t)| \leq C|t|^{\rho-1 / 2} e^{-\pi|t| / 2} .
$$

We start by upper bounding via the triangle inequality. Then we upper bound $|T(s)|^{-m}$ : noting that, for any $s=\rho+i t, T(s) \neq 0$ and

$$
\left|T\left(s+i \frac{2 \pi}{\log (p / q)}\right)\right|=|T(s)|,
$$

there exists some $L(\rho)$ such that

$$
|T(s)|^{-m} \leq|L(\rho)|^{-m} .
$$

The formula (23) immediately gives an exponentially decaying upper bound on $\Gamma(s+1)$ which holds for sufficiently large $|t|$.

In order to bound, for each $m$, the $n^{\prime}$ sum, we split it into two pieces: an initial part, to be bounded using (23), and a tail part, which we bound using the exponential decay of $e^{-\Theta\left(n^{\prime}\right)} \eta_{n^{\prime}, m}$. More specifically, provided $m \leq\lceil\sqrt{|t|}\rceil$, we split the $n^{\prime}$ sum as follows:

$$
\sum_{n^{\prime}=m}^{\infty} e^{-\Theta\left(n^{\prime}\right)} \eta_{n^{\prime}, m} \frac{\Gamma\left(n^{\prime}+s\right)}{\Gamma\left(n^{\prime}+1\right)}=\sum_{n^{\prime}=m}^{\lceil\sqrt{|t|}\rceil} e^{-\Theta\left(n^{\prime}\right)} \eta_{n^{\prime}, m} \frac{\Gamma\left(n^{\prime}+s\right)}{\Gamma\left(n^{\prime}+1\right)}+\sum_{n^{\prime}=\lceil\sqrt{|t|}\rceil+1}^{\infty} e^{-\Theta\left(n^{\prime}\right)} \eta_{n^{\prime}, m} \frac{\Gamma\left(n^{\prime}+s\right)}{\Gamma\left(n^{\prime}+1\right)} .
$$

Bounding the initial sum: To upper bound the initial sum of (24), we apply (23), which gives us an upper bound of

$$
C|t|^{\lceil\sqrt{|t|}\rceil+\rho-1 / 2} e^{-\pi|t| / 2} \sum_{n^{\prime}=m}^{\lceil\sqrt{|t|}\rceil} \frac{e^{-\Theta\left(n^{\prime}\right)}\left|\eta_{n^{\prime}, m}\right|}{\Gamma\left(n^{\prime}+1\right)} .
$$

Since $\left|\eta_{n^{\prime}, m}\right| \leq c_{1} n^{\prime c_{2}}$, we have that

$$
\sum_{n^{\prime}=m}^{\lceil\sqrt{|t|}\rceil} \frac{e^{-\Theta\left(n^{\prime}\right)}\left|\eta_{n^{\prime}, m}\right|}{\Gamma\left(n^{\prime}+1\right)} \leq \sum_{n^{\prime}=0}^{\infty} \frac{e^{-\Theta\left(n^{\prime}\right)}}{n^{\prime} !}=\Theta(1)
$$

Furthermore,

$$
|t|^{\lceil\sqrt{|t|}\rceil}=e^{\lceil\sqrt{|t|}\rceil \log |t|}=e^{o(|t|)}
$$

so that, clearly, for sufficiently large $|t|$, the initial sum can be upper bounded by some $e^{-\gamma_{1}|t|}$ whenever $m \leq\left\lceil\sqrt{|t|}\right.$. For larger $m$, the initial sum is 0 , by the property (22) of $\eta_{n^{\prime}, m}$.

Bounding the tail sum: To upper bound the tail sum of (24), we note that $e^{-\Theta\left(n^{\prime}\right)}\left|\eta_{n^{\prime}, m}\right|$ can be upper bounded by $e^{-g\left(n^{\prime}\right)}$, for some $g\left(n^{\prime}\right)$ which is monotone increasing and which satisfies $g\left(n^{\prime}\right)=\Omega\left(n^{\prime}\right)$. Then

$$
e^{-g\left(n^{\prime}\right) / 2} \leq e^{-g(\lceil\sqrt{|t|}\rceil+1) / 2},
$$

by the fact that $e^{-g\left(n^{\prime}\right)}$ is monotone decreasing with respect to $n^{\prime}$. Furthermore,

$$
\left|\Gamma\left(n^{\prime}+s\right)\right| \leq\left|\Gamma\left(n^{\prime}+\rho\right)\right|,
$$


noting that $n^{\prime}$ is larger than $-\rho$ provided that $|t|$ is sufficiently large, so that $\left|\Gamma\left(n^{\prime}+\rho\right)\right|<\infty$. Thus, we can upper bound the tail sum of (24) by

$$
e^{-g(\lceil\sqrt{|t|}\rceil+1) / 2} \cdot \sum_{n^{\prime}=\lceil\sqrt{|t|}\rceil+1}^{\infty} e^{-g\left(n^{\prime}\right) / 2}\left|\eta_{n^{\prime}, m}\right|\left|\frac{\Gamma\left(n^{\prime}+\rho\right)}{\Gamma\left(n^{\prime}+1\right)}\right| .
$$

Collecting the contribution of the tail sum over all $m$ gives and upper bound of

$$
e^{-g(\lceil\sqrt{|t|}\rceil+1) / 2} \sum_{m=0}^{\infty}|T(s)|^{-m} \sum_{n^{\prime}=\lceil\sqrt{|t|}\rceil+1}^{\infty} e^{-g\left(n^{\prime}\right) / 2}\left|\eta_{n^{\prime}, m}\right|\left|\frac{\Gamma\left(n^{\prime}+\rho\right)}{\Gamma\left(n^{\prime}+1\right)}\right| .
$$

As noted earlier, we can upper bound $|T(s)|^{-m}$ by $|L(\rho)|^{-m}$. Furthermore, since $\eta_{n^{\prime}, m}=0$ when $m \geq n^{\prime}$, we can replace the lower index of the $n^{\prime}$ sum by $m$ to get an upper bound, yielding

$$
\sum_{m=0}^{\infty}|L(\rho)|^{-m} \sum_{n^{\prime}=m}^{\infty} e^{-g\left(n^{\prime}\right) / 2}\left|\eta_{n^{\prime}, m}\right|\left|\frac{\Gamma\left(n^{\prime}+\rho\right)}{\Gamma\left(n^{\prime}+1\right)}\right|,
$$

and by Lemma 3 , this is less than $\infty$, so $O(1)$ with respect to $|t|$. Thus, the total contribution of the tail sum is at most

$$
O\left(e^{-g(\lceil\sqrt{|t|}\rceil+1) / 2}\right) .
$$

As an immediate consequence (in particular because $\mu_{m, j}=0$ for $j \geq m$ ), we see that there is some $r>0$ for which

$$
\left|X_{k}(s)\right| \leq e^{-\gamma|t|^{r}}
$$

as $|t| \rightarrow \infty$ with $s=\rho+i t$.

\subsubsection{Inverting the Mellin transform}

To extract asymptotics for $z \rightarrow \infty$ of $\tilde{G}_{k}(z)$, we next evaluate the inverse Mellin transform of $G_{k}^{*}(s)$ :

$$
\tilde{G}_{k}(z)=\frac{1}{2 \pi i} \int_{c-i \infty}^{c+i \infty} G_{k}^{*}(s) z^{-s} \mathrm{~d} s=\frac{1}{2 \pi i} \int_{c-i \infty}^{c+i \infty} z^{-s} \Gamma(s+1) A_{k}(s) T(s)^{k} \mathrm{~d} s,
$$

where $-k-1<c<\infty$. When $k$ is in the range specified by the theorem, the asymptotics of this integral are dictated by the saddle points of the function $s \mapsto z^{-s} T(s)^{k}$ (note that both factors are tending to infinity as $|z|=n \rightarrow \infty)$. Thus, we choose the line of integration to coincide with the real solution $\rho=\rho(\alpha)$ of the saddle point equation

$$
\frac{\mathrm{d}}{\mathrm{d} s}[k \log T(s)-s \log z]=0 .
$$

Solving this, we get $s=\rho$ as defined in the statement of the theorem:

$$
\rho(\alpha)=-\frac{1}{\log (p / q)} \log \left(\frac{\alpha \log (1 / q)-1}{1-\alpha \log (1 / p)}\right) .
$$

Furthermore, since $\left|T\left(\rho+i t_{j}\right)\right|=|T(\rho)|$ for all $j \in \mathbb{Z}$, we find that the integrand has infinitely many regularly spaced saddle points $\rho_{j}$ with real part equal to $\rho$, which will turn out to lead to 
a fluctuating factor in $\tilde{G}_{k}(z)$. A fact about $\rho$ should be noted here: as we vary $\alpha$ from $\frac{1}{\log (1 / q)}$ to $\frac{1}{\log (1 / p)}, \rho$ goes from $\infty$ to $-\infty$, which corresponds to the boundaries for the range we consider: at the left endpoint, the numerator inside the logarithm is 0 , while at the right, the denominator is.

Our evaluation of (26) then proceeds as follows: we split the contour into two parts: the outer tails, which we will show to be negligible, and the central region. By the negligibility of the outer tails, we then need only consider the contribution of the finitely many saddle points in the central region. Around each such saddle point, we consider a small region in which the tails are negligible, and the central part is approximable by a Gaussian integral. Summing the contributions gives the desired result. We note that we follow the high-level plan of [17], but the bounding of the tails and the evaluation of the central regions crucially relies on the information that we have derived about the behavior of $A_{k}(s) \Gamma(s+1)$.

In what follows, we define

$$
J_{k}(n, s)=n^{-s} T(s)^{k} A_{k}(s) \Gamma(s+1) .
$$

Furthermore, we define the outer tails, the inner tails, and the central parts as, respectively,

$$
\begin{aligned}
C^{O} & =C_{n, k}^{O}=\frac{1}{2 \pi} \int_{|t| \geq \sqrt{\log n}} J_{k}(n, \rho+i t) \mathrm{d} t \\
C_{j}^{I O} & =C_{n, k, j}^{I O}=\frac{1}{2 \pi} \int_{k^{-2 / 5} \leq\left|t-t_{j}\right| \leq \frac{\pi}{\log (p / q)}} J_{k}(n, \rho+i t) \mathrm{d} t \\
C_{j}^{I I} & =C_{n, k, j}^{I I}=\frac{1}{2 \pi} \int_{\left|t-t_{j}\right| \leq k^{-2 / 5}} J_{k}(n, \rho+i t) \mathrm{d} t .
\end{aligned}
$$

We let $j_{0}=\Theta(\sqrt{\log n})$ denote the index of the furthest saddle point from the real axis in the central region. Finally, we denote by $C_{j}^{I}$ the contribution of the region around the $j$ th saddle point, including its center and tails:

$$
C_{j}^{I}=C_{j}^{I O}+C_{j}^{I I}
$$

The choice of $\Theta\left(k^{-2 / 5}\right)$ for the central region lengths is a result of the following heuristic: for the application of the saddle point method to evaluating the contributions of the integral on the central regions, in writing $n^{-s} T(s)^{k}$ in the form $e^{d_{k}(t)}$ and applying the Taylor expansion of $d_{k}(t)$ around $t=t_{j}$, we want to ensure that the remainder term, which is $O\left(k\left(t-t_{j}\right)^{3}\right)$, is negligible, while the previous term, $\frac{d_{k}^{\prime \prime}(t)}{2}\left(t-t_{j}\right)^{2}=\Theta\left(k\left(t-t_{j}\right)^{2}\right)$, is not. That is, if we assume that $\left|t-t_{j}\right|=O\left(k^{-\delta}\right)$, for some $\delta$, then it is natural to enforce the constraint that $1-2 \delta>0$ and $1-3 \delta<0$; that is, $\delta \in(1 / 3,1 / 2)$, and it is easy to check that $\delta=2 / 5$ satisfies these constraints.

\section{OUTER TAILS}

First, we bound the outer tails. To be precise, we have the following claim.

Lemma 5 (Bound on $C^{O}$ ). There exist positive constants $\gamma, r$ such that

$$
C^{O}=O\left(n^{-\rho} T(\rho)^{k} e^{-\gamma(\log n)^{r / 2}}\right),
$$

uniformly in $n$ and $k$.

In the proof, we will need the following elementary lemma about $T(s)$ near the saddle points.

Lemma 6. For $\left|t-t_{j}\right| \leq \frac{\pi}{\log (p / q)}$,

$$
|T(\rho+i t)| \leq T(\rho) e^{-c_{0}\left(t-t_{j}\right)^{2}}
$$


where $c_{0}$ is given by

$$
c_{0}=\frac{2 p^{-\rho} q^{-\rho} \log (p / q)^{2}}{\pi^{2}(T(\rho))^{2}} .
$$

We omit the proof, which can be found in [17].

Proof of Lemma 5. To bound $\left|n^{-s} T(s)^{k}\right|$, we apply Lemma 6 to conclude that

$$
\left|n^{-s} T(s)^{k}\right| \leq n^{-\rho} T(\rho)^{k} .
$$

Thus, we are left with the task of upper bounding

$$
\int_{|t| \geq \sqrt{\log n}}\left|X_{k}(\rho+i t)\right| \mathrm{d} t
$$

which we write as

$$
\sum_{j:\left|t_{j}\right| \geq \sqrt{\log n}} \int_{\left|t-t_{j}\right| \leq \frac{\pi}{\log (p / q)}}\left|X_{k}(\rho+i t)\right| \mathrm{d} t .
$$

Next, we apply (25) and the assumption that $n \rightarrow \infty$ to conclude that (29) can be upper bounded, for some positive constants $\gamma$ and $r$, by

$$
O\left(\sum_{j:\left|t_{j}\right| \geq \sqrt{\log n}} e^{-\gamma\left|t_{j}\right|^{r}}\right)=O\left(e^{-\gamma(\log n)^{r / 2}}\right) .
$$

Thus,

$$
\int_{|t| \geq \sqrt{\log n}} J_{k}(n, \rho+i t) \mathrm{d} t=O\left(n^{-\rho} T(\rho)^{k} e^{-\gamma(\log n)^{r / 2}}\right) .
$$

This completes the proof of Lemma 5 that the outer tails are negligible.

INNER TAILS

Now we move to the central region. We first show that the tails in the central region are negligible.

Lemma 7 (Inner tails are negligible). The total contribution of the inner tails is

$$
\sum_{|j| \leq j_{0}} C_{j}^{I O}=O\left(n^{-\rho} T(\rho)^{k} k^{-1 / 10} e^{-c_{0} k^{1 / 5}}\right),
$$

which is negligible compared to $\tilde{G}_{k}(n)$.

First, we recall a simple fact [22] bounding incomplete Gaussian integrals, which will be useful when we apply Lemma 6 to handle the contribution of $T(\rho+i t)$.

$$
x \geq 0 \Longrightarrow \int_{x}^{\infty} e^{-c t^{2}} \mathrm{~d} t \leq \frac{1}{2 c x} e^{-c x^{2}} .
$$


Proof of Lemma \%. We will start by bounding the contribution of $C_{j}^{I O}$ for an arbitrary $j$. Since $X_{k}(\rho+i t)$ is uniformly bounded above by a constant whenever $|t| \leq \sqrt{\log (n)}$ as a consequence of (25), we can pull it out of the integral defining $C_{j}^{I O}$. Next, we use the equality $\left|n^{-(\rho+i t)}\right|=n^{-\rho}$ and Lemma 6 to conclude that

$$
C_{j}^{I O}=O\left(n^{-\rho} T(\rho)^{k} \int_{k^{-2 / 5} \leq\left|t-t_{j}\right| \leq \frac{\pi}{\log (p / q)}} e^{-c_{0}\left(t-t_{j}\right)^{2} k} \mathrm{~d} t\right) .
$$

Applying the substitution $t \mapsto t-t_{j}$ in the above integral and extending the domain of integration from $\left[k^{-2 / 5}, \frac{\pi}{\log (p / q)}\right]$ to $\left[k^{-2 / 5}, \infty\right)$, we are left with the incomplete Gaussian integral

$$
\int_{k^{-2 / 5}}^{\infty} e^{-c_{0} k t^{2}} \mathrm{~d} t
$$

Applying the inequality (30) then yields

$$
C_{j}^{I O}=O\left(n^{-\rho} T(\rho)^{k} k^{-3 / 5} e^{-c_{0} k^{1 / 5}}\right) .
$$

Since this bound is uniform in $j$ for $|j| \leq j_{0}$, the total contribution of the inner tails is then given by

$$
O\left(n^{-\rho} T(\rho)^{k} k^{-1 / 10} e^{-c_{0} k^{1 / 5}}\right)
$$

that is, we multiplied by $k^{1 / 2}$. Now, the factor $k^{-1 / 10} e^{-c_{0} k^{1 / 5}}=o\left(k^{-1 / 2}\right)$, so that the inner tails are negligible compared to the entire integral.

\section{Central Region}

We now show that a quadratic approximation to the integrand holds in the central region around each saddle point.

Lemma 8 (Central region). We have, for each $|j| \leq j_{0}$,

$$
C_{j}^{I I}=\Gamma\left(\rho+1+i t_{j}\right) A_{k}\left(\rho+i t_{j}\right) e^{-i t_{j} \log \left(p^{k} n\right)} \frac{n^{-\rho} T(\rho)^{k}}{\sqrt{2 \pi \kappa_{*}(\rho) k}}\left(1+O\left(k^{-1 / 2}\right)\right) .
$$

Proof. Suppose that, for some $j,\left|t-t_{j}\right| \leq k^{-2 / 5}$, and let $s=\rho+i t$ and $s_{j}=\rho+i t_{j}$.

First, we develop the quadratic approximation to $n^{-s} T(s)^{k}$. Noting that $n^{-\rho-i t} T(\rho+i t)^{k}=$ $e^{(-\rho-i t) \log n+k \log (T(\rho+i t))}$, we define

$$
d_{k}(t)=-(\rho+i t) \log n+k \log T(\rho+i t)
$$

We note that

$$
n^{-\rho-i t}=n^{-\rho-i\left(t-t_{j}\right)-i t_{j}}=e^{-i t_{j} \log n} n^{-\rho-i\left(t-t_{j}\right)}
$$

and

$$
\begin{aligned}
T(\rho+i t)^{k} & =T\left(\rho+i\left(t-t_{j}\right)+i t_{j}\right)^{k} \\
& =\left(p^{-\left(\rho+i\left(t-t_{j}\right)\right)} p^{-i t_{j}}+q^{-\left(\rho+i\left(t-t_{j}\right)\right)} q^{-i t_{j}}\right)^{k} \\
& =p^{-i t_{j} k}\left(p^{-\left(\rho+i\left(t-t_{j}\right)\right)}+q^{-\left(\rho+i\left(t-t_{j}\right)\right)}\left(\frac{p}{q}\right)^{i t_{j}}\right)^{k} \\
& =p^{-i t_{j} k} T\left(\rho+i\left(t-t_{j}\right)\right)^{k}
\end{aligned}
$$


so that

$$
d_{k}(t)=-\left(\rho+i\left(t-t_{j}\right)\right) \log n+k \log T\left(\rho+i\left(t-t_{j}\right)\right)-i t_{j} \log n(1+\alpha \log p) .
$$

Then we have, by Taylor expanding $d_{k}(t)$ around $t_{j}$,

$$
d_{k}(t)=d_{k}\left(t_{j}\right)+\frac{d_{k}^{\prime \prime}\left(t_{j}\right)}{2}\left(t-t_{j}\right)^{2}+r_{k}^{(3)}\left(t_{j}\right)
$$

where we note that $d_{k}^{\prime}\left(t_{j}\right)=0$, since $t_{j}$ is a saddle point of $d_{k}(t)$, and $r_{k}^{(3)}\left(t_{j}\right)$ is the remainder corresponding to the Taylor polynomial of degree 2. Taking derivatives, we have

$$
d_{k}^{\prime \prime}(t)=k \cdot \frac{T^{\prime}\left(\rho+i\left(t-t_{j}\right)\right)^{2}-T\left(\rho+i\left(t-t_{j}\right)\right) T^{\prime \prime}\left(\rho+i\left(t-t_{j}\right)\right)}{T\left(\rho+i\left(t-t_{j}\right)\right)^{2}} .
$$

We also need $d_{k}^{(3)}(t)$ for the analysis of the remainder term, and it can be similarly explicitly computed, but it suffices to note that, since it is a function of $t-t_{j}$, evaluating it at $t=t_{j}$ gives a function which is a constant with respect to $j$ and $t$. Thus, considering the Lagrange form of $r_{k}^{(3)}\left(t_{j}\right)$ shows that it is $O\left(k\left(t-t_{j}\right)^{3}\right)=O\left(k^{5 / 5-6 / 5}\right)=O\left(k^{-1 / 5}\right)$, so the remainder is negligible in comparison to the first and second terms. Evaluating $d_{k}\left(t_{j}\right)$ and $d_{k}^{\prime \prime}\left(t_{j}\right)$ gives

$$
d_{k}\left(t_{j}\right)=-\rho \log n+k \log T(\rho)-i t_{j} \log \left(p^{k} n\right)
$$

and

$$
d_{k}^{\prime \prime}\left(t_{j}\right)=k \frac{T^{\prime}(\rho)^{2}-T(\rho) T^{\prime \prime}(\rho)}{T(\rho)^{2}}
$$

which we will denote by $k \kappa_{*}(\rho)$.

We consider separately the cases where $|j| \rightarrow \infty$ arbitrarily slowly and where $|j|$ remains bounded.

$j$ tending to $\infty$ : First, we need to show that $\Gamma(s+1) A_{k}(s)=X_{k}(s) \sim X_{k}\left(s_{j}\right)$ for $s$ in this range (recalling that $t_{j}=O(\sqrt{\log n})$ and $\left|s-s_{j}\right|=O\left(k^{-2 / 5}\right)$ ). Recall that $X_{k}(s)$ is a double sum of the form

$$
\sum_{m=0}^{k} T(s)^{-m} \sum_{n^{\prime} \geq m} T\left(-n^{\prime}\right)\left(\mu_{n^{\prime}, m}-\mu_{n^{\prime}, m-1}\right) \frac{\Gamma\left(n^{\prime}+s\right)}{\Gamma\left(n^{\prime}+1\right)} .
$$

By the analysis in Lemma 3, we have that the $m$ th term of this sum is $O\left(e^{-\Theta(m)}\right)$, so that if $m \gg \log k=\Theta(\log \log n)$, then these terms are negligible (i.e., they decay superpolynomially in $k$ ).

It remains to handle the terms with $m=O(\log k)$. First, we note that, by the Taylor expansion for $T(s)$,

$$
\begin{aligned}
T(s)^{-m} & =T\left(s_{j}\right)^{-m}\left(1+O\left(T^{\prime}\left(s_{j}\right) / T\left(s_{j}\right) k^{-2 / 5}\right)\right)^{-m} \\
& =T\left(s_{j}\right)^{-m}\left(1+O\left(k^{-2 / 5}\right)\right)^{-m} \\
& =T\left(s_{j}\right)^{-m}\left(1+O\left(k^{-2 / 5} \log k\right)\right) \\
& \sim T\left(s_{j}\right)^{-m} .
\end{aligned}
$$

Now we must determine the contribution of the inner sum, indexed by $n^{\prime}$. We split it into initial and final parts:

$$
\sum_{n^{\prime}=m}^{n^{\prime \prime}} T\left(-n^{\prime}\right)\left(\mu_{n^{\prime}, m}-\mu_{n^{\prime}, m-1}\right) \frac{\Gamma\left(n^{\prime}+s\right)}{\Gamma\left(n^{\prime}+1\right)}+\sum_{n^{\prime}>n^{\prime \prime}} T\left(-n^{\prime}\right)\left(\mu_{n^{\prime}, m}-\mu_{n^{\prime}, m-1}\right) \frac{\Gamma\left(n^{\prime}+s\right)}{\Gamma\left(n^{\prime}+1\right)},
$$


where $n^{\prime \prime}$ is to be determined. We will handle the initial part using the Taylor expansion for the $\Gamma$ function, and the latter we show to be negligible thanks to the $T\left(-n^{\prime}\right)$ factor.

For the initial sum, we recall that the first-order Taylor polynomial for $\Gamma\left(n^{\prime}+s\right)$ around $n^{\prime}+s_{j}$ is given by

$$
\Gamma\left(n^{\prime}+s\right)=\Gamma\left(n^{\prime}+s_{j}\right)+\Gamma^{\prime}\left(n^{\prime}+s_{j}\right)\left(s-s_{j}\right) .
$$

Now, recall that, for any $x$ for which $\Gamma(x)$ is analytic,

$$
\Gamma^{\prime}(x)=\Gamma(x) \psi(x),
$$

where $\psi(x)$ is the digamma function (see [1]). Continuing the derivation in (31),

$$
\Gamma\left(n^{\prime}+s\right)=\Gamma\left(n^{\prime}+s_{j}\right)\left(1+\psi\left(n^{\prime}+s_{j}\right)\left(s-s_{j}\right)\right)
$$

and the second term in parentheses is $o(1)$ if $\psi\left(n^{\prime}+s_{j}\right)=o\left(k^{2 / 5}\right)$. We have that, as $x \rightarrow \infty$ with $x$ bounded away from the negative real axis, $\psi(x) \sim \log x$, so that it suffices to note that (since $\left.s_{j}=O(\sqrt{\log n})\right)$

$$
n^{\prime}=o\left(e^{k^{2 / 5}}\right) \Longrightarrow\left|n^{\prime}+s_{j}\right|=o\left(e^{k^{2 / 5}}\right)
$$

Thus, we choose, say, $n^{\prime \prime}=e^{k^{1 / 5}}$ to split the sum, and for the initial sum, we have

$$
\Gamma\left(n^{\prime}+s\right)=\Gamma\left(n^{\prime}+s_{j}\right)\left(1+O\left(k^{-1 / 5}\right)\right) .
$$

Because of the presence of $T(-n)$, the final sum is $O\left(e^{-\Theta\left(e^{k^{1 / 5}}\right)}\right)$. Putting all of this together, we get that

$$
X_{k}(s) \sim X_{k}\left(s_{j}\right)
$$

as claimed.

Then we have

$$
\begin{aligned}
C_{j}^{I I} & =\frac{1}{2 \pi i} \int_{\left|t-t_{j}\right| \leq k^{-2 / 5}} X_{k}(\rho+i t) n^{-\rho-i t} T(\rho+i t)^{k} \mathrm{~d} t \\
& =(1+o(1)) X_{k}\left(\rho+i t_{j}\right) \frac{1}{2 \pi i} \int_{\left|t-t_{j}\right| \leq k^{-2 / 5}} e^{d_{k}\left(t_{j}\right)+\frac{d_{k}^{\prime \prime}\left(t_{j}\right)}{2}\left(t-t_{j}\right)^{2}+O\left(k^{-1 / 5}\right)} \mathrm{d} t
\end{aligned}
$$

and invoking the saddle point method on the remaining integral gives a contribution of

$$
C_{j}^{I I}=e^{-i t_{j} \log \left(p^{k} n\right)} X_{k}\left(\rho+i t_{j}\right) \frac{n^{-\rho} T(\rho)^{k}}{\sqrt{2 \pi k \kappa_{*}(\rho)}}\left(1+O\left(k^{-1 / 2}\right)\right)
$$

for $j$ tending to $\infty$ with $j \leq j_{0}=O(\sqrt{\log n})$.

$|j|$ bounded: Meanwhile, for $|j|$ bounded above by any fixed $C$, we have that $X_{k}(s) \sim X_{k}\left(s_{j}\right)$, by continuity. Invoking the saddle point method again on this integral gives the same contribution as (32). 


\section{Collecting estimates}

To complete the computation, we add all of the contributions of the saddle points:

$$
\begin{aligned}
& \tilde{G}_{k}(n)=\sum_{|j| \leq j_{0}} C_{j}^{I}+O\left(n^{-\rho} T(\rho)^{k} e^{-\sqrt{\log n}}\right) \\
& =\sum_{|j| \leq j_{0}} \Gamma\left(\rho+1+i t_{j}\right) A_{k}\left(\rho+i t_{j}\right) e^{-i t_{j} \log \left(p^{k} n\right)} \frac{n^{-\rho} T(\rho)^{k}}{\sqrt{2 \pi \kappa_{*}(\rho) k}}\left(1+O\left(k^{-1 / 2}\right)\right) \\
& +O\left(n^{-\rho} T(\rho)^{k} e^{-\gamma \sqrt{\log n}}\right) \\
& =H_{k}\left(\rho, \log _{p / q}\left(p^{k} n\right)\right) \frac{n^{-\rho} T(\rho)^{k}}{\sqrt{2 \pi \kappa_{*}\left(\rho_{n, k}\right) k}}\left(1+O\left(k^{-1 / 2}\right)\right)
\end{aligned}
$$

where $H_{k}(\rho, x)$ is given by

$$
H_{k}(\rho, x)=\sum_{j \in \mathbb{Z}} A_{k}\left(\rho+i t_{j}\right) \Gamma\left(\rho+1+i t_{j}\right) e^{-2 j \pi i x} .
$$

Note that we extended the limits of the $j$ sum to $\pm \infty$ by virtue of the exponential decay of $X_{k}(s)$ on vertical lines.

The dependence of $H_{k}(\rho, x)$ on $k$ must now be analyzed. This is the content of the following claim.

Lemma 9 (Convergence of $H_{k}(\rho, x)$ ). The limit

$$
\lim _{k \rightarrow \infty} H_{k}(\rho, x)
$$

exists and is equal to $H(\rho, x)$. Furthermore, the convergence is uniform for $(\rho, x)$ in any compact set.

Proof. We can write

$$
\lim _{k \rightarrow \infty} H_{k}(\rho, x)=\lim _{k \rightarrow \infty} \lim _{j^{\prime} \rightarrow \infty} \sum_{j=-j^{\prime}}^{j^{\prime}} A_{k}\left(\rho+i t_{j}\right) \Gamma\left(\rho+1+i t_{j}\right) e^{-2 \pi j i x},
$$

and our task is to show that the limits exist and can be interchanged. It suffices, by the dominated convergence theorem (which holds when the functions to be dominated take values in a Banach space), to show that $A_{k}(s)$ converges pointwise for all $s$, and then that the sum

$$
\sum_{j \in \mathbb{Z}} A\left(s_{j}\right) \Gamma\left(s_{j}+1\right) e^{-2 \pi i j x}
$$

converges absolutely for all $\rho, x$. Pointwise convergence of $A_{k}(s)$ was already established in Corollary 1. The absolute convergence of (33) is a simple consequence of (25) and the ratio test. Note that, implicitly, we've used

$$
\sum_{j \in \mathbb{Z}}\left|A\left(s_{j}\right) \Gamma\left(s_{j}+1\right)\right|
$$

as our bounding function.

To complete the derivation, we must recover asymptotics of $\mu_{n, k}$ from $\tilde{G}_{k}(z)$. See Appendix 4.3 .1 for the justification of this step. 


\subsection{Proof of Theorem 2}

Here we prove (13) of Theorem 2. The proof of the superexponentially decaying bound (16) can be found in Appendix 4.2.4. The derivation of asymptotics for the variance runs along lines very similar to that of the expected value, and the technical machinery developed in the proof of Theorem 1 is applicable here. The main difference lies in the de-Poissonization step, where we use Theorem 6 of [10]. The resulting expansion for $V_{n, k}$ involves $n \tilde{G}_{k}^{\prime}(n)^{2}$, which we must show to be negligible in order to get the claimed result. This we can easily do via the Cauchy integral formula for derivatives and our knowledge of the asymptotics of $\tilde{G}_{k}(n)$.

The Poisson variance is given by $\tilde{V}_{k}(z)=\tilde{C}_{k}(z)+\tilde{G}_{k}(z)-\left(\tilde{G}_{k}(z)\right)^{2}$, where $\tilde{C}_{k}(z)$ is the Poisson transform of $c_{n, k}$, the second factorial moment of $B_{n, k}$. Our first task is to derive functional equations for $\tilde{C}_{k}(z)$ and $\tilde{V}_{k}(z)$. We recall the recurrence for $c_{n, k}$, which holds for $n \geq 2$ and $k \geq 1$ :

$$
c_{n, k}=T(-n) c_{n, k}+\sum_{j=1}^{n-1}\left(\begin{array}{l}
n \\
j
\end{array}\right) p^{j} q^{n-j}\left(c_{j, k-1}+c_{n-j, k-1}+2 \mu_{j, k-1} \mu_{n-j, k-1}\right),
$$

with $c_{n, k}=0$ whenever $n<2, k<1$, or $k \geq n$. Introducing the exponential generating function $C_{k}(z)$ for the sequence $c_{n, k}$, we get a functional equation:

$$
\begin{aligned}
C_{k}(z)= & C_{k-1}(p z) e^{q z}+C_{k-1}(q z) e^{p z}+2 G_{k-1}(p z) G_{k-1}(q z) \\
& +\left[C_{k}(p z)-C_{k-1}(p z)\right]+\left[C_{k}(q z)-C_{k-1}(q z)\right],
\end{aligned}
$$

valid for $k \geq 1$, with initial condition $C_{0}(z)=0$.

Multiplying by $e^{-z}$ on both sides to form the Poisson transform $\tilde{C}_{k}(z)$ of the sequence $c_{n, k}$ and abbreviating using the linear operators $L[\cdot]$ and $T[\cdot]$, we get the following functional equation:

$$
\tilde{C}_{k}(z)=L[\tilde{C}]_{k-1}(z)+T[\tilde{C}]_{k}(z)+2 \tilde{G}_{k-1}(p z) \tilde{G}_{k-1}(q z),
$$

with $\tilde{C}_{0}(z)=0$.

Now we derive a functional equation for $\tilde{V}_{k}(z)$. Applying the equations for $\tilde{G}_{k}(z)$ and $\tilde{C}_{k}(z)$ to the definition of $\tilde{V}_{k}(z)$, we have

$$
\begin{aligned}
\tilde{V}_{k}(z)= & L[\tilde{C}]_{k-1}(z)+T[\tilde{C}]_{k}(z)+2 \tilde{G}_{k-1}(p z) \tilde{G}_{k-1}(q z) \\
& +L[\tilde{G}]_{k-1}(z)+T[\tilde{G}]_{k}(z)-\left(L[\tilde{G}]_{k-1}(z)+T[\tilde{G}]_{k}(z)\right)^{2}
\end{aligned}
$$

To handle the squared term in the previous equation, we need the following identity on $L[\cdot]$ :

$$
\left(L[f]_{k}(z)\right)^{2}=L\left[f^{2}\right]_{k}(z)+2 f_{k}(p z) f_{k}(q z) .
$$

Now, expanding the squared term, we get

$$
\begin{aligned}
\left(L[\tilde{G}]_{k-1}(z)+T[\tilde{G}]_{k}(z)\right)^{2} & =L[\tilde{G}]_{k-1}(z)^{2}+2 L[\tilde{G}]_{k-1}(z) T[\tilde{G}]_{k}(z)+T[\tilde{G}]_{k}(z)^{2} \\
& =L\left[\tilde{G}^{2}\right]_{k-1}(z)+2 \tilde{G}_{k-1}(p z) \tilde{G}_{k-1}(q z)+2 L[\tilde{G}]_{k-1}(z) T[\tilde{G}]_{k}(z)+T[\tilde{G}]_{k}(z)^{2}
\end{aligned}
$$

Substituting this last expression into the derivation of $\tilde{V}_{k}(z)$ above gives, after noting the cancellation of terms and applying linearity of $L[\cdot]$ and the definition of $\tilde{V}_{k-1}(z)$,

$$
\tilde{V}_{k}(z)=L[\tilde{V}]_{k-1}(z)+T[\tilde{C}+\tilde{G}]_{k}(z)-T[G]_{k}(z)^{2}-2 L[\tilde{G}]_{k-1}(z) T[\tilde{G}]_{k}(z)
$$


Adding and subtracting $L[\tilde{G}]_{k-1}(z)^{2}$ and applying the functional equation for $\tilde{G}_{k}(z)$, this reduces to

$$
\tilde{V}_{k}(z)=L[\tilde{V}]_{k-1}(z)+T[\tilde{C}+\tilde{G}]_{k}(x)+L[\tilde{G}]_{k-1}(z)^{2}-\tilde{G}_{k}(z)^{2}=L[\tilde{V}]_{k-1}(z)+\tilde{W}_{k, V}(z),
$$

where $\tilde{W}_{k, V}(z)$ is given by

$$
\tilde{W}_{k, V}(z)=T[\tilde{C}+\tilde{G}]_{k}(z)+L[\tilde{G}]_{k-1}(z)^{2}-\tilde{G}_{k}(z)^{2} .
$$

The above recurrence holds for $k \geq 1$. To derive the initial condition, we write

$$
\tilde{V}_{0}(z)=\tilde{C}_{0}(z)+\tilde{G}_{0}(z)-\left(\tilde{G}_{0}(z)\right)^{2}=z e^{-z}-z^{2} e^{-2 z} .
$$

Now, to solve this equation, the plan is again to apply the Mellin transform and invert using the saddle point method. We have, by the same derivation as in the expected value case, $V_{k}^{*}(s)=$ $T(s)^{k} \Gamma(s+1) B_{k}(s)$, where we define

$$
B_{k}(s)=1-(s+1) 2^{-(s+2)}+\sum_{j=1}^{k} T(s)^{-j} \frac{W_{j, V}^{*}(s)}{\Gamma(s+1)},
$$

with $W_{k, V}^{*}(s)$, the Mellin transform of $\tilde{W}_{k, V}(z)$, given by

$$
\begin{aligned}
& W_{j, V}^{*}(s)=\sum_{n^{\prime} \geq j} \frac{\Gamma\left(s+n^{\prime}\right)}{n^{\prime} !}\left[\left(c_{n^{\prime}, j}-c_{n^{\prime}, j-1}+\mu_{n^{\prime}, j}-\mu_{n^{\prime}, j-1}\right) T\left(-n^{\prime}\right)+T(s) 2^{-\left(s+n^{\prime}\right)} \sum_{m=0}^{n^{\prime}} \mu_{m, j-1} \mu_{n^{\prime}-m, j-1}\right. \\
&\left.+2 \sum_{m=0}^{n^{\prime}} \mu_{m, j-1} \mu_{n^{\prime}-m, j-1} p^{m} q^{n^{\prime}-m}-2^{-\left(n^{\prime}+s\right)} \sum_{m=0}^{n^{\prime}} \mu_{m, j} \mu_{n^{\prime}-m, j}\right]
\end{aligned}
$$

We have the following information about the singularities and zeros of $V_{k}^{*}(s)$.

Theorem 6 (Special points of $V_{k}^{*}(s)$ ). The function $B_{k}(s)$ is entire, with zeros at $s=-1, \ldots,-k$. The function $V_{k}^{*}(s)$ is analytic except possibly at the integers less than $-k$, so that its fundamental strip contains $I_{k}=\{s \in \mathbb{C}: \Re(s) \in(-k-1, \infty)\}$.

This is analogous to Theorem 4, and the proof is along similar lines. In particular, we can demonstrate the claim about the fundamental strip corresponding to $\tilde{V}_{k}(z)$ by estimates of $\tilde{V}_{k}(z)$ at $z \rightarrow 0$ and $z \rightarrow \infty$, and the formula for $V_{k}^{*}(s)$ throughout $I_{k}$ is demonstrated, as in the expected value case, by an analytic continuation argument.

We first need some bounds on $\tilde{C}_{k}(z)$ and $\tilde{V}_{k}(z)$ as $z \rightarrow \infty$ inside a cone around the positive real axis. The exponentially decaying upper bound on $\left|\tilde{V}_{k}(z)\right|$ as $z \rightarrow \infty$ is analogous to Lemma 1, part (ii).

Lemma 10 (Bounds on $\tilde{C}_{k}(z)$ and $\tilde{V}_{k}(z)$ as $\left.z \rightarrow \infty\right)$. Let $\theta$ be as in Lemma 1. Then

(i) For every $\epsilon>0$, there are some $R>0$ and $C=C(R)>0$ such that, for $|z|>R$ with $z \in \mathcal{C}(\theta)$

$$
\left|\tilde{C}_{k}(z)\right| \leq C|z|^{2+\epsilon}
$$

for any $k \geq 0$. 
(ii) For any fixed $C>0$, there exists a constant $C^{\prime}>0$ such that, for all $j \leq C$ and $z \in \mathcal{C}(\theta)$,

$$
\left|\tilde{V}_{j}(z)\right| \leq C^{\prime}|z|^{3} e^{-q^{C}|z| \cos (\theta)}
$$

The proof is in Appendix 4.2.3.

We now have enough to prove Theorem 6.

Proof of Theorem 6. Existence and entireness of $B_{k}(s)$ follow easily from Lemma 2: we simply note that the inequalities $\mu_{m, j} \leq m$ for all $m$ and $c_{m, j} \leq m^{2}$ for all $m$ imply the necessary bound on the growth of the terms of the series.

Now we estimate the growth of $\tilde{V}_{k}(z)$ as $z \rightarrow 0$ and $z \rightarrow \infty$ in order to establish the existence of the Mellin transform in a nonempty strip. Applying Lemma 10, part (ii) gives a sufficient bound as $z \rightarrow \infty$. Next, recalling the definition of $\tilde{V}_{k}(z)$ in terms of $\tilde{C}_{k}(z)$ and $\tilde{G}_{k}(z)$, it is sufficient to determine the behavior of $\tilde{C}_{k}(z)$ as $z \rightarrow 0$. By the initial conditions, $c_{n, k}=0$ for $k \geq n$, so that $\tilde{C}_{k}(z)=O\left(z^{k+1}\right)$ as $z \rightarrow 0$. This, in turn, implies the same estimate for $\tilde{V}_{k}(z)$, since $\tilde{G}_{k}(z)-\left(\tilde{G}_{k}(z)\right)^{2}=O\left(z^{k+1}\right)$. We have thus established the existence and analyticity of $V_{k}^{*}(s)$, for any $k \geq 0$, in the strip $I_{k}$.

The rest of the proof is very similar to that in the expected value case, so we omit it.

\subsubsection{Mellin inversion}

The inversion of the Mellin transform $V_{k}^{*}(s)$ runs along lines very similar to those in the expected value case. In fact, the saddle point computation used to recover $\tilde{G}_{k}(z)$ generalizes in a straightforward manner to the variance case, provided that we can show that Lemma 4 applies with $B_{k}(s)$ in place of $A_{k}(s)$. Finally, the application of the dominated convergence theorem is justified if we can apply Lemma 3, with $U(s)$ in the lemma corresponding to the sum in the definition of $\Gamma(s+1) B(s)$. All of this boils down to showing that each term inside the brackets in the definition of $W_{j, V}^{*}(s)$ can be written as a product of a function which is bounded along vertical lines, $e^{-\Omega\left(n^{\prime}\right)}$, and a number $\eta_{n^{\prime}, j}$ satisfying the conditions required by Lemma 3 , with $\eta_{n^{\prime}, j}=0$ when $j \geq n^{\prime}$. The uniform boundedness condition on $\left|\eta_{n^{\prime}, j}\right|$ is easily verified using the fact that $c_{n^{\prime}, j} \leq n^{\prime 2}$ and $\mu_{n^{\prime}, j} \leq n^{\prime}$ for all $n^{\prime}$ and $j$, and factors of the form $e^{c s}$, for any constant $c$, are uniformly bounded on vertical lines:

$$
\begin{aligned}
\left(c_{n^{\prime}, j}-c_{n^{\prime}, j-1}+\mu_{n^{\prime}, j}-\mu_{n^{\prime}, j-1}\right) T(-n) & \leq\left(n^{\prime 2}+n^{\prime}\right) e^{-\Omega\left(n^{\prime}\right)} \\
2^{-n^{\prime}} \sum_{m=0}^{n^{\prime}} \mu_{m, j-1} \mu_{n^{\prime}-m, j-1} & \leq 2^{-n^{\prime}}\left(n^{\prime}+1\right) n^{\prime 2} \\
\sum_{m=0}^{n^{\prime}} \mu_{m, j-1} \mu_{n^{\prime}-m, j-1} p^{m} q^{n^{\prime}-m} & \leq\left(n^{\prime}+1\right) n^{\prime 2} p^{n^{\prime}} .
\end{aligned}
$$

Moreover, $\eta_{n^{\prime}, j}=0$ for $j \geq n^{\prime}$ because of the same property for $c_{n^{\prime}, j}$ and $\mu_{n^{\prime}, j}$.

In order to verify the superexponential decay condition required of $\eta_{n^{\prime}, j}$, we apply (16) and the analogous bound for $\mu_{n, k}$. By the same analysis as in the expected value case, (16) then implies that

$$
\tilde{V}_{k}(n)=M\left(\rho(\alpha), \log _{p / q}\left(p^{k} n\right)\right) \cdot \frac{n^{-\rho(\alpha)} T(\rho(\alpha))^{k}}{\sqrt{2 \pi \kappa_{*}(\rho(\alpha)) k}}\left(1+O\left(k^{-1 / 2}\right)\right) .
$$

The de-Poissonization step can be found in Appendix 4.3.2. 


\subsection{Proof of Theorem 3}

To prove the normal limit law, we apply Lévy's continuity theorem:

Theorem 7 (Lévy). Let $X_{n}$ be a sequence of discrete random variables with probability-generating functions $G_{n}(u)=\mathbb{E}\left[u^{X_{n}}\right]$, mean $\mu_{n}$, and variance $\sigma_{n}^{2}>0$. If

$$
\lim _{n \rightarrow \infty} e^{\tau \mu_{n} / \sigma_{n}} G_{n}\left(e^{\tau / \sigma_{n}}\right)=e^{\tau^{2} / 2}
$$

for all $\tau=$ it and $-\infty<t<\infty$ then

$$
\frac{X_{n}-\mu_{n}}{\sigma_{n}} \stackrel{D}{\rightarrow} \mathcal{N}(0,1)
$$

The goal, then, is to determine the behavior of $Q_{n, k}(u)$ for large $n$ and $u$ close to 1 (i.e., $u=e^{\tau / \sigma_{n, k}}$, where $\tau$ is any fixed imaginary number). To do this, we again use the Poisson transform, defining

$$
Q_{k}(u, z)=\sum_{m \geq 0} Q_{m, k}(u) \frac{z^{m}}{m !}, \quad \quad \tilde{Q}_{k}(u, z)=e^{-z} Q_{k}(u, z) .
$$

We then have the functional equation

$$
Q_{j}(u, x)=Q_{j-1}(u, p x) Q_{j-1}(u, q x)+\left(Q_{j}-Q_{j-1}\right)(u, p x)+\left(Q_{j}-Q_{j-1}\right)(u, q x),
$$

with initial condition $Q_{0}(u, x)=e^{x}-x(1-u)$.

Defining $\tilde{l}_{k}(u, z)=\log Q_{k}(u, z)$ induces the functional equation (for $j \geq 1$ )

$$
\tilde{l}_{j}(u, x)=\tilde{l}_{j-1}(u, p x)+\tilde{l}_{j-1}(u, q x)+\tilde{h}_{j}(u, x),
$$

where

$$
\tilde{h}_{j}(u, x)=\log \left(1+\frac{T[\tilde{Q}]_{j}(u, x)}{\tilde{Q}_{j-1}(u, p x) \tilde{Q}_{j-1}(u, q x)}\right),
$$

with initial condition $\tilde{l}_{0}(u, x)=x+\log \left(1+(u-1) x e^{-x}\right)$. Regarding well definedness of $\tilde{l}_{k}(u, z)$, we must demonstrate that the logarithm which constitutes it is well defined for $u$ in a bounded neighborhood of 1 and $z$ in a small enough cone around the positive real axis, for any $k \geq 0$. It is sufficient to show that $Q_{k}(u, z)$ is bounded away from 0 . This follows easily from the argument of Lemma 12 below (we note that, though this is stated and proven later, there is no circular logic here, as the lemma's proof does not depend on the existence of $\tilde{l}_{k}(u, z)$ ), which can be extended to require only that $w$ be close enough to 1 but fixed (since we assume that $w-1 \rightarrow 0$ arbitrarily slowly).

Now, since we are going to take $u \stackrel{n \rightarrow \infty}{\longrightarrow} 1$, we Taylor expand $\tilde{l}_{k}(u, z)$ with respect to $u$ around 1 , which gives

$$
\tilde{l}_{k}(u, z)=z+\tilde{G}_{k}(z)(u-1)+\frac{\tilde{V}_{k}(z)-\tilde{G}_{k}(z)}{2}(u-1)^{2}+\frac{(u-1)^{3}}{3 !} R\left[\tilde{l}_{k}(u, z),\right.
$$

and the task now is to show that the last term is negligible with respect to the second two. Setting $u=e^{\tau / \sigma_{n, k}}$ with $\tau=i t$, with $t \in \mathbb{R}$, we have

$$
u-1=\frac{\tau}{\sigma_{n, k}}+\frac{\tau^{2}}{2 \sigma_{n, k}^{2}}+O\left(\sigma_{n, k}^{-3}\right),
$$


and after substituting this into the Taylor expansion for $\tilde{l}_{k}(u, z)$ and using the fact that $\tilde{G}_{k}(z)=$ $\Theta\left(\sigma_{n, k}^{2}\right)$ (valid for $z \rightarrow \infty$ inside a cone around the positive real axis), we get

$$
\tilde{l}_{k}(u, z)=z+\frac{\tau}{\sigma_{n, k}} \tilde{G}_{k}(z)+\frac{\tau^{2}}{2 \sigma_{n, k}^{2}} \tilde{V}_{k}(z)+O\left(\sigma_{n, k}^{-1}\right)+\frac{\tau^{3}}{3 ! \sigma_{n, k}^{3}} R\left[\tilde{l}_{k}(u, z) .\right.
$$

Since $V_{k}(z)=\Theta\left(\sigma_{n, k}^{2}\right)$, the third term is $\Theta(1)$. Thus, the goal is to show that the last term is $o(1)$ (in fact, we prove that $\left|R[\tilde{l}]_{k}(u, z)\right|=O\left(n^{\beta(\alpha)}\right)=O\left(\sigma_{n, k}^{2}\right)$, so that the last term is $O\left(\sigma_{n, k}^{-1}\right)=o(1)$ ).

In order to do this, the plan is to apply an exact formula for the remainder of a truncated Taylor series, derived via Cauchy's integral formula.

Lemma 11 (Integral representation of Taylor remainder formula). Suppose $f(z)$ is a function which is analytic in some region $\Omega$ containing the point $\xi$, and let $j$ be some non-negative integer. Define $f_{j}(z)$ to be the Taylor polynomial of degree $j$ around $\xi$, and define $(z-\xi)^{j+1} R_{\xi, j}(z)=f(z)-f_{j}(z)$ to be the corresponding remainder. Then, for any $z$ inside the disc of convergence of the Taylor series of $f$ at $\xi$,

$$
R_{\xi, j}(z)=\frac{1}{2 \pi i} \oint_{\mathcal{C}} \frac{f(w)}{(w-\xi)^{j+1}(w-z)} \mathrm{d} w,
$$

where $\mathcal{C}$ is any circle inside $\Omega$ centered at $\xi$ surrounding $z$.

Applying this to the Taylor series of $\tilde{l}_{k}(u, z)$ with $j$ and $\xi$ in the lemma set to 2 and 1 , respectively, we get

$$
\frac{1}{3 !} R[\tilde{l}]_{k}(u, z)=\frac{1}{2 \pi i} \oint_{\mathcal{C}} \frac{\tilde{l}_{k}(w, z)}{(w-1)^{3}(w-u)} \mathrm{d} w
$$

Here, we choose $\mathcal{C}$ to be a circle centered around 1 and such that $|w-1| \gg|u-1|$, so that, in particular, $u$ remains inside the region enclosed by $\mathcal{C}$. We will require that $|w-1|$ tends to 0 sufficiently slowly. We next derive a useful representation for $\tilde{l}_{k}(w, z)$ in order to bound this integral. Iterating the functional equation (36), we get

$\tilde{l}_{k}(w, z)=\sum_{j=0}^{k}\left(\begin{array}{c}k \\ j\end{array}\right)\left(p^{j} q^{k-j} z+\log \left(1+(w-1) p^{j} q^{k-j} z e^{-p^{j} q^{k-j} z}\right)\right)+\sum_{j=1}^{k} \sum_{m=0}^{k-j}\left(\begin{array}{c}k-j \\ m\end{array}\right) \tilde{h}_{j}\left(w, p^{m} q^{k-j-m} z\right)$,

and plugging this into (38) finally gives $\frac{1}{3 !} R\left[\tilde{l}_{k}(u, z)=R_{1, k}(u, z)+R_{2, k}(u, z)\right.$, where we've defined

$$
\begin{aligned}
& R_{1, k}(u, z)=\sum_{j=0}^{k}\left(\begin{array}{c}
k \\
j
\end{array}\right) \frac{1}{2 \pi i} \oint_{\mathcal{C}} \frac{p^{j} q^{k-j} z+\log \left(1+(w-1) p^{j} q^{k-j} z e^{-p^{j} q^{k-j} z}\right)}{(w-1)^{3}(w-u)} \mathrm{d} w \\
& R_{2, k}(u, z)=\sum_{j=0}^{k} \sum_{m=0}^{k-j}\left(\begin{array}{c}
k-j \\
m
\end{array}\right) \frac{1}{2 \pi i} \oint_{\mathcal{C}} \frac{\tilde{h}_{j}\left(w, p^{m} q^{k-j-m} z\right)}{(w-1)^{3}(w-u)} \mathrm{d} w .
\end{aligned}
$$

We define

$$
S_{j}(w, x)=\frac{\left(Q_{j}-Q_{j-1}\right)(w, p x)+\left(Q_{j}-Q_{j-1}\right)(w, q x)}{Q_{j-1}(w, p x) Q_{j-1}(w, q x)},
$$

so that we can write

$$
\tilde{h}_{j}(w, x)=\log \left(1+S_{j}(w, x)\right) .
$$


We will show below that both $R_{1, k}(u, z)$ and $R_{2, k}(u, z)$ are $O\left(n^{\beta(\alpha)}\right)$, so that $(u-1)^{3} R\left[\tilde{l}_{k}(u, z) / 3 !=\right.$ $O\left(\sigma_{n, k}^{-1}\right)$, which is negligible with respect to the first three terms in the expansion $(37)$ of $\tilde{l}_{k}(u, z)$. This is sufficient to establish the central limit theorem in the Poisson model. In the subsections corresponding to $R_{1, k}(u, z)$ and $R_{2, k}(u, z)$, respectively, we first give roadmaps of the proofs of the bounds and then delve into the details of the derivations.

\subsubsection{Bounding $R_{1, k}(u, z)$}

Roadmap of the bound on $R_{1, k}(u, z)$

To bound $R_{1, k}(u, z)$, the idea is first to observe that the logarithm in the integrand of each term of the summation is given by $\log (1+g(w, x))$, where $g(w, x)=o(1)$, so that a natural idea to evaluate the integral is to use the residue theorem, and computing residues can by done via a Laurent expansion around $w=1$ (the contribution of the term outside the logarithm vanishes). This leaves us with the task of bounding

$$
\sum_{j=0}^{k}\left(\begin{array}{l}
k \\
j
\end{array}\right)\left(p^{j} q^{k-j} z\right)^{3} e^{-3 p^{j} q^{k-j} z}
$$

We do this by writing the summand as $e^{\nu(j)}$, for some function $\nu$, and using elementary calculus to determine the maximum of $\nu(j)$ in the interval $[0, k]$. This tells us that the largest term is $\beta(\alpha) \log n(1+o(1))$, which concludes the bounding of $R_{1, k}(u, z)$.

\section{Details of the derivation}

First, the residue theorem tells us that the term

$$
\oint_{\mathcal{C}} \frac{p^{j} q^{k-j} z}{(w-1)^{3}(w-u)} \mathrm{d} w
$$

vanishes, and we need not consider it for the rest of the derivation. Now, note that the logarithm in the $j$ th term can be written as

$$
\log \left(1+(w-1) x_{k, j}(z) e^{-x_{k, j}(z)}\right),
$$

where we've defined $x_{k, j}(z)=p^{j} q^{k-j} z$. Then

$$
|w-1|\left|x_{k, j}(z) e^{-x_{k, j}(z)}\right|=|w-1| x_{k, j}(|z|) e^{-x_{k, j}(|z|) \cos (\arg (z))}
$$

Since the function $x \mapsto x e^{-c x}$ is bounded for real $x, c \geq 0$ (which can be seen by elementary calculus), and since $|w-1| \stackrel{n \rightarrow \infty}{\longrightarrow} 0$ and $|\arg (z)|$ is less than and bounded away from $\pi / 2$, we have that the above expression is $O(|w-1|)=o(1)$, so that we can expand (39) as a Taylor series around $w=1$, which gives

$$
\log \left(1+(w-1) x_{k, j} e^{-x_{k, j}(z)}\right)=\sum_{\ell=1}^{\infty}(-1)^{\ell+1}(w-1)^{\ell}\left(p^{j} q^{k-j} z\right)^{\ell} e^{-\ell p^{j} q^{k-j} z}
$$

Plugging this into the integral corresponding to the $j$ th term of $R_{1, k}(u, z)$ and defining $r(n)$ to be the radius of $\mathcal{C}$ (and, hence, equal to $|w-1|)$ gives

$$
\left(1+r(n)^{-1}(u-1)\right) \frac{1}{2 \pi i} \oint_{\mathcal{C}} \sum_{\ell=1}^{\infty} \frac{(-1)^{\ell+1}(w-1)^{\ell}\left(p^{j} q^{k-j} z\right)^{\ell} e^{-\ell p^{j} q^{k-j} z}}{(w-1)^{4}} \mathrm{~d} w .
$$


Here, the $\left(1+r(n)^{-1}(u-1)\right)=1+o(1)$ outside comes from approximating $w-u$ in the denominator of the integrand by $w-1$, which is valid since we've chosen $u-1=o(w-1)$. Applying the Cauchy residue theorem to evaluate this integral gives

$$
\left(1+(u-1) r(n)^{-1}\right)\left(p^{j} q^{k-j} z\right)^{3} e^{-3 p^{j} q^{k-j} z}
$$

Plugging this in as the $j$ th term of $R_{1, k}(u, z)$ reduces the problem to bounding

$$
\sum_{j=0}^{k}\left(\begin{array}{l}
k \\
j
\end{array}\right)\left(p^{j} q^{k-j} z\right)^{3} e^{-3 p^{j} q^{k-j} z}
$$

Using Stirling's formula for the binomial coefficient, we write the $j$ th term as $e^{\nu(j)}$, where we define

$$
\begin{aligned}
\nu(j) & =k \log k-j \log j-(k-j) \log (k-j) \\
& +\frac{1}{2}(\log k-\log j-\log (k-j))+3 j \log p+3(k-j) \log q+3 \log z-3 p^{j} q^{k-j} z+O(1) .
\end{aligned}
$$

Our goal is to find $j$ which maximizes this expression. We first observe that the terms for which $j=o(\log n)$ or $j=k(1-o(1))$ contribute negligibly: in such cases, $\left(\begin{array}{l}k \\ j\end{array}\right) \leq \frac{k^{j}}{j !}$ and $\left(\begin{array}{l}k \\ j\end{array}\right) \leq \frac{k^{k-j}}{(k-j) !}$. This results in an upper bound of $e^{o(\log n)}=n^{o(1)}$ for $\left(\begin{array}{l}k \\ j\end{array}\right)$, and the factor $\left(p^{j} q^{k-j} z\right)^{3} e^{-3 p^{j} q^{k-j} z}$ is, as previously mentioned, bounded. Thus, the contribution of such factors is $n^{o(1)}$, so that it remains to handle terms for which $j=\Theta(\log n)$. Taking the derivative of $\nu$ with respect to $j$, we get

$$
\begin{aligned}
\nu^{\prime}(j) & =-1-\log j-\frac{1}{2 j}+\frac{1}{2(k-j)}+\frac{k}{k-j}+\log (k-j)-\frac{j}{k-j}+3 \log (p / q)-3 q^{k} n \log (p / q)(p / q)^{j} \\
& =\log \left(\frac{k-j}{j}\right)+3 \log (p / q)-3 q^{k} n(p / q)^{j} \log (p / q)+O\left((\log n)^{-1}\right) .
\end{aligned}
$$

Setting $j=K(\alpha) k$ for $K(\alpha) \in(0,1)$ to be determined under the conditions that $\nu^{\prime}(j)=0$ and $K(\alpha)$ as a function of $n$ remains bounded away from 0 and 1 (since $j=\Theta(k)$ ), we find that the first two terms of $\nu^{\prime}(j)$ are $O(1)$, so that $\nu^{\prime}(j)=0$ only if the third term is also $O(1)$. This boils down to requiring

$$
q^{k} n(p / q)^{j}=e^{k \log q+\log n+K(\alpha) k \log (p / q)}=O(1) .
$$

Equivalently,

$$
k \log q+\log n+K(\alpha) k \log (p / q)=\log n(\alpha \log q+1+K(\alpha) \alpha \log (p / q))=O(1),
$$

so that, in particular, we require

$$
\alpha \log q+1+K(\alpha) \alpha \log (p / q)=0
$$

This gives

$$
K(\alpha)=\frac{\alpha \log (1 / q)-1}{\alpha \log (p / q)} .
$$

It is easily checked by taking another derivative of $\nu$ that this gives a global maximum. We then have that the maximum term in $R_{1, k}(u, z)$ is given by $n^{\chi(\alpha)}$, where $\chi(\alpha)=\alpha \cdot h(K(\alpha))$, with, again, $h(x)=x \log (1 / x)+(1-x) \log (1 /(1-x))$. After some algebra, we see that $\chi(\alpha)=\beta(\alpha)$, so that $R_{1, k}(u, z)=O\left(n^{\beta(\alpha)}\right)$, which is negligible when multiplied by $(u-1)^{3}$. 


\subsubsection{Bounding $R_{2, k}(u, z)$}

Roadmap of the bound on $R_{2, k}(u, z)$

Handling $R_{2, k}(u, z)$ is more difficult. For this, we need good upper and lower bounds, respectively, on the numerator and denominator of $S_{j}(w, x)$. The plan is as follows. We split the outer sum into three ranges, corresponding to $j=o(\log n), j=\Omega(\log n)$ and $k-j=\Omega(\log n)$, and $j \sim k$. The first and third range can be handled via a somewhat crude (though still technically difficult) upper bound (Corollary 2 below) on the terms of the $m$ sum which reduces the analysis of the $j$ sum in those ranges to that of a sum similar to $R_{1, k}(u, z)$. The middle range, on the other hand, requires more finesse. We split the inner sum into two parts: those terms for which $p^{m} q^{k-j-m} z=O(\log n)$ and those for which it is $\gg \log n$. For the former range, we find that we must refine the crude upper bound on $\left|S_{j}(w, x)\right|$ to account for the cancellation inherent in its definition, which requires that we take advantage of the fact that $j=\Omega(\log n)$ and that we have a superexponentially decaying upper bound on $\mu_{m, j}$ for $m=\Theta(j)$. For the latter range, we can use the coarser upper bound.

Details of the derivation: Estimates on $Q_{j}(w, x)$ and $S_{j}(w, x)$

We first proceed to prove several facts about the growth of $Q_{j}(w, x)$, which will be useful in the handling of the first and third ranges mentioned above. We start by giving a precise estimate of $\left|Q_{j}(w, x)\right|$ which is uniform in $j$, in the case where $|x|=O(1)$.

Lemma 12 (Uniform estimate of $\left|Q_{j}(w, x)\right|$ for bounded $|x|$ ). Suppose $|x| \leq C$ for some fixed positive $C$, and $w \sim e^{i t / g(n)}$, where $g(n) \stackrel{n \rightarrow \infty}{\longrightarrow} \infty$ and $t \in \mathbb{R}$. Then, uniformly for $j \leq k$,

$$
Q_{j}(w, x) \sim e^{x}
$$

Proof. We split the series defining $Q_{j}(w, x)$ into two parts: for any function $f(n)=o(g(n))$ which is also $\gg 1$,

$$
Q_{j}(w, x)=\sum_{m=0}^{f(n)} \mathbb{E}\left[w^{B_{m, j}}\right] \frac{x^{m}}{m !}+\sum_{m>f(n)} \mathbb{E}\left[w^{B_{m, j}}\right] \frac{x^{m}}{m !} .
$$

We will show that $\mathbb{E}\left[w^{B_{m, j}}\right]=1+o(1)$ in the first sum and that the second sum is negligible. To see the claim for the first sum, we note that since $m \leq f(n)$ and $B_{m, j} \in[0, m]$,

$$
w^{B_{m, j}} \sim e^{i t B_{m, j} / g(n)}=1+\frac{i t B_{m, j}}{g(n)}+O\left((f(n) / g(n))^{2}\right)=1+O(f(n) / g(n))=1+o(1),
$$

where the $o(\cdot)$ is uniform in $j$.

To show the claim for the second sum, we upper bound its absolute value using the triangle inequality, giving

$$
\sum_{m>f(n)} \mathbb{E}\left[|w|^{B_{m, j}}\right] \frac{|x|^{m}}{m !}
$$

Next, we define $\xi=1+|w-1|>1$ and note that, since $B_{m, j} \leq m$,

$$
\xi^{B_{m, j}} \leq \xi^{m}
$$

Since $|x|$ is bounded above by $C$, we get that this is the tail of a convergent series, and $f(n) \rightarrow 0$, so that the second sum is $o(1)$, so negligible with respect to the first, and this completes the proof.

Next we upper bound the differences $\left|Q_{j}(w, x)-Q_{j-1}(w, x)\right|$. 
Lemma 13 (Uniform upper bound on differences). Suppose $|x| \leq C$ for some fixed positive $C$, and $w \sim e^{i t / g(n)}$, where $g(n) \stackrel{n \rightarrow \infty}{\longrightarrow} \infty$ and $t \in \mathbb{R}$. Then, uniformly for $j \leq k$,

$$
\left(Q_{j}-Q_{j-1}\right)(w, x)=O\left(|x| e^{|x|} / g(n)\right) .
$$

Proof. The idea of this proof is very similar to that of Lemma 12. We choose an appropriate function $f(n)$ such that $1 \ll f(n) \ll g(n)$ (for reasons to be seen below, we will also require that $\left.g(n)=o\left(e^{\Theta(f(n) \log f(n))}\right)\right)$, and we split the series defining $\left(Q_{j}-Q_{j-1}\right)(w, x)$ into an initial and final part:

$$
\left(Q_{j}-Q_{j-1}\right)(w, x)=\sum_{m=0}^{f(n)} \mathbb{E}\left[w^{B_{m, j}}-w^{B_{m, j-1}}\right] \frac{x^{m}}{m !}+\sum_{m>f(n)} \mathbb{E}\left[w^{B_{m, j}}-w^{B_{m, j-1}}\right] \frac{x^{m}}{m !} .
$$

Bounding the initial sum: Again using the fact that $0 \leq m \leq f(n)=o(g(n))$ as in the previous lemma, we find that

$$
\mathbb{E}\left[w^{B_{m, j}}-w^{B_{m, j-1}}\right]=O(m / g(n)),
$$

uniformly in $j$. Plugging this into the first sum, we get an upper bound of

$$
O\left(g(n)^{-1}\right) \sum_{m=0}^{f(n)} m \frac{x^{m}}{m !}=O\left(g(n)^{-1}|x| e^{|x|}\right),
$$

and, since $|x| \leq C$, this is $O\left(g(n)^{-1}\right)$.

Bounding the final sum: It remains to bound the final sum. Recalling the definition of $\xi$ in the proof of the previous lemma, the second sum is upper bounded by

$$
2 \sum_{m>f(n)} \frac{\xi^{m}|x|^{m}}{m !}
$$

Factoring out $(\xi|x|)^{f(n)+1} /(f(n)+1)$ !, we get the expression

$$
2 \frac{(\xi|x|)^{f(n)+1}}{(f(n)+1) !} \sum_{m \geq f(n)+1} \frac{(\xi|x|)^{m-f(n)-1}}{m(m-1) \cdots(f(n)+2)} .
$$

Noting that the denominator consists of $m-(f(n)+2)+1=m-f(n)-1$ factors greater than $f(n)+1$, we can lower bound it (thereby upper bounding the whole expression) by $(m-f(n)-1)$ !, so that the sum is upper bounded by $e^{\xi|x|}$. Thus, applying the assumed bound on $|x|$, the second sum is upper bounded by

$$
\frac{2(C \xi)^{f(n)+1}}{(f(n)+1) !} e^{\xi C}
$$

which we note is upper bounded by

$$
e^{-\Theta(f(n) \log f(n))},
$$

due to the factorial in the denominator. Choosing $f(n)$ to be, say, $f(n)=\sqrt{g(n)}$, we see that this is

$$
o\left(e^{-\Theta(\sqrt{g(n) \log g(n)})}\right),
$$

so that the second sum is negligible compared to the first. 
Finally, we extend Lemma 12 to the case where $x \stackrel{n \rightarrow \infty}{\longrightarrow} \infty$. For the purposes of this lemma, we restrict our attention to a cone around the positive real axis.

Lemma 14 (Uniform estimate of $\left|Q_{j}(w, x)\right|$ for unbounded $|x|$ inside a cone). Let $w \sim e^{i t / g(n)}$ for some $g(n) \stackrel{n \rightarrow \infty}{\longrightarrow} \infty$ and $t \in \mathbb{R}$. Then there exists some $\theta_{0} \in(0, \pi / 2)$ such that, for any $\theta \in\left(0, \theta_{0}\right)$, uniformly for $j \leq k$,

$$
\left|Q_{j}(w, x)\right|=e^{|x| \cos (\arg (x))(1+o(1))},
$$

as $x \rightarrow \infty$ inside $\mathcal{C}(\theta)$.

Proof. We will approach this by proving an upper and a lower bound on $\left|\tilde{Q}_{j}(w, x)\right|$; that is, for some functions $a(x)$ and $b(x)$ satisfying certain growth properties (to be explained), we will prove that, for all sufficiently small positive constants $\epsilon$, for large enough $|x|$,

$$
e^{\epsilon b(|x|)} \leq\left|\tilde{Q}_{j}(w, x)\right| \leq e^{\epsilon a(|x|)} .
$$

Provided that $a(|x|)$ and $b(|x|)=O(|x|)$ as $x \rightarrow \infty$, we will then have

$$
\begin{array}{r}
\left|Q_{j}(w, x)\right|=\left|e^{x}\right|\left|\tilde{Q}_{j}(w, x)\right|=e^{|x| \cos (\arg (x))}\left|\tilde{Q}_{j}(w, x)\right|, \\
e^{|x| \cos (\arg (x))+\epsilon b(|x|)} \leq\left|Q_{j}(u, x)\right| \leq e^{|x| \cos (\arg (x))+\epsilon a(|x|)},
\end{array}
$$

so that

$$
\left|Q_{j}(u, x)\right|=e^{|x| \cos (\arg (x))+o(|x|)} .
$$

We propose

$$
a(x)=x-1 \quad b(x)=-a(x),
$$

As before, we derive a useful bound on $\left|\tilde{Q}_{\tilde{j}}(w, x)\right|$ by setting $\xi=1+|w-1|$, so that $\mathbb{E}\left[|w|^{B_{m, j}}\right] \leq$ $\xi^{m}$, and plugging this into the definition of $\widetilde{Q}_{j}(w, x)$ gives

$$
\left|\tilde{Q}_{j}(w, x)\right| \leq e^{|x|(\xi-\cos \theta)} .
$$

We will use this inequality in what follows.

We now prove the claimed bounds on $\left|\tilde{Q}_{j}(w, x)\right|$, for arbitrarily small fixed $\epsilon$. We do this by induction on $j$. The idea is as follows: we have, by Lemma 12 , that $Q_{j}(w, x) \sim e^{x}$, uniformly for all $j \leq k$, when $x=O(1)$. This particularly implies that there is some large enough fixed $x_{*}$ in the cone for which the claimed inequalities on $\tilde{Q}_{j}\left(w, x_{*}\right)$ hold. In particular, they hold for $x$ inside the cone with $|x| \in\left(\left|x_{*}\right|,\left|x_{*}\right| / q\right]$, again for all $j \leq k$. In order to prove the inequalities for the rest of the cone (i.e., $\left.|x| \in\left(\left|x_{*}\right| / q, \infty\right)\right)$, we then apply the recurrence and the inductive hypothesis.

\section{Base case}

Recall that $\tilde{Q}_{0}(w, x)=1-x e^{-x}(1-w)=1+o(1)$, where the $o$ is with respect to $x \rightarrow \infty$. The claimed decay of the second term is because $\left|x e^{-x}\right|$ remains bounded inside the cone, while $1-w \stackrel{n \rightarrow \infty}{\longrightarrow} 0$. Then, $e^{\epsilon a(|x|)}=e^{\epsilon(|x|-1)} \rightarrow \infty$. Furthermore, $e^{\epsilon b(|x|)}=e^{-\epsilon(|x|-1)} \rightarrow 0$. Thus, for sufficiently large $|x|$ (depending on $\epsilon$ ), the claimed inequality holds.

\section{Inductive step}

For the induction, we assume that the claim is true for all $h<j$, and we prove it for $j$. By the observation above, the inequalities hold for $\tilde{Q}_{j}(w, x)$ when $|x| \in\left(\left|x_{*}\right|,\left|x_{*}\right| / q\right]$, and it remains to establish that they hold for larger $|x|$, so we assume from here onward that $|x| \in\left(\left|x_{*}\right| / q, \infty\right)$. 
Recall the recurrence for $\tilde{Q}_{j}(w, x)$, which holds for all $j \geq 1$ :

$$
\tilde{Q}_{j}(w, x)=\tilde{Q}_{j-1}(w, p x) \tilde{Q}_{j-1}(w, q x)+e^{-q x}\left(\tilde{Q}_{j}-\tilde{Q}_{j-1}\right)(w, p x)+e^{-p x}\left(\tilde{Q}_{j}-\tilde{Q}_{j-1}\right)(w, q x) .
$$

\section{Upper bound inductive step}

We first handle the induction step for the upper bound. The first step is to upper bound $\left|\tilde{Q}_{j}(w, x)\right|$ using the triangle inequality. Next, we handle the product: by the inductive hypothesis (applicable here because $\left.\left|x_{*}\right|<|q x|<|p x|\right)$, we have

$$
\left|\tilde{Q}_{j-1}(w, p x) \tilde{Q}_{j-1}(w, q x)\right| \leq e^{\epsilon(a(p|x|)+a(q|x|))}=e^{\epsilon a(|x|)-\epsilon},
$$

where the equality is easy algebra based on the definition of $a(x)$. To handle the terms of the form

$$
\left|e^{-(1-c) x}\right|\left(\left|\tilde{Q}_{j}(w, c x)\right|+\left|\tilde{Q}_{j-1}(w, c x)\right|\right),
$$

we apply the bound (41) to both terms. This gives

$$
\left|e^{-(1-c) x}\right|\left(\left|\tilde{Q}_{j}(w, c x)\right|+\left|\tilde{Q}_{j-1}(w, c x)\right|\right) \leq 2 e^{|c x|(\xi-\cos \theta)-(1-c)|x| \cos \theta}=2 e^{|x|(c \xi-\cos \theta)} \leq 2 e^{|x|(p \xi-\cos \theta)} .
$$

Provided $|\xi-1|$ is sufficiently small (with respect to $\cos \theta$ ) and $|\theta|$ sufficiently small with respect to $p$, the quantity in the exponent is negative and bounded away from 0 . This can be done by making $n$ sufficiently large.

Then,

$$
\begin{aligned}
\left|\tilde{Q}_{j}(w, x)\right| & \leq e^{\epsilon(a(p|x|)+a(q|x|))}\left(1+4 e^{|x|(p \xi-\cos \theta)-\epsilon(a(p|x|)-a(q|x|))}\right) \\
& \leq e^{\epsilon(a(p|x|)+a(q|x|))}\left(1+4 e^{|x|(p \xi-\cos \theta)}\right)
\end{aligned}
$$

where the second inequality is because $a(c|x|)>0$ when $|x|$ is large enough (depending only on $c$ ). The factor in parentheses can be written as

$$
e^{\log \left(1+4 e^{|x|(p \xi-\cos \theta)}\right)}=e^{4 e^{|x|(p \xi-\cos \theta)(1+o(1))}},
$$

since, by a previous observation, $p \xi-\cos \theta<0$. Thus, the upper bound becomes

$$
e^{\epsilon a(|x|)-\epsilon+4 e^{|x|(p \xi-\cos \theta)}} \text {. }
$$

Since the second term is a constant and the third term decays exponentially with respect to $|x| \rightarrow \infty$, we can further upper bound by $e^{\epsilon a(|x|)}$, provided $|x|$ is sufficiently large. This concludes the proof of the upper bound.

\section{Lower bound inductive step}

We now give the inductive step of the lower bound. First, we use the lower bound version of the triangle inequality (and we note that, for $c>0, e^{-c|x| \cos (\arg (x))} \leq e^{-c|x| \cos \theta}$, since $\cos \theta \leq$ $\cos (\arg (x))$ and the function $y \mapsto e^{y}$ is monotone increasing with respect to $\left.y\right)$ :

$$
\begin{aligned}
\left|\tilde{Q}_{j}(w, x)\right| & \geq\left|\tilde{Q}_{j-1}(w, p x) \tilde{Q}_{j-1}(w, q x)\right| \\
& -e^{-p|x| \cos \theta}\left|\tilde{Q}_{j}-\tilde{Q}_{j-1}\right|(w, q x)-e^{-q|x| \cos \theta}\left|\tilde{Q}_{j}-\tilde{Q}_{j-1}\right|(w, p x) .
\end{aligned}
$$

We apply the inductive hypothesis to the product (justified by the same reasoning as in the upper bound proof) to get

$$
\left|\tilde{Q}_{j-1}(w, p x) \tilde{Q}_{j-1}(w, q x)\right| \geq e^{\epsilon(b(p|x|)+b(q|x|))} .
$$


For the other two terms, we require an upper bound on expressions of the form

$$
e^{-(1-c)|x| \cos \theta}\left|\tilde{Q}_{j}-\tilde{Q}_{j-1}\right|(w, c x) .
$$

Applying the triangle inequality and then the bound (41), we get that the above expression is upper bounded by

$$
2 e^{-(1-c)|x| \cos \theta} e^{c|x|(\xi-\cos \theta)}=2 e^{|x|(c \xi-\cos \theta)} \leq 2 e^{|x|(p \xi-\cos \theta)} .
$$

Thus, we get

$$
\left|\tilde{Q}_{j}(w, x)\right| \geq e^{\epsilon(b(p|x|)+b(q|x|))}\left(1-4 e^{|x|(p \xi-\cos \theta)-\epsilon(b(p|x|)+b(q|x|))}\right) .
$$

Provided $0<\epsilon<\cos \theta-p \xi$ (which can hold if we choose $\theta$ close enough to 0 ), we can see by substituting in the definition of $b(x)$ that there exists a positive number $\tau$ (depending only on $\epsilon, \theta, p, \xi)$ such that

$$
\left|\tilde{Q}_{j}(w, x)\right| \geq e^{\epsilon(b(p|x|)+b(q|x|)}\left(1-4 e^{-\tau|x|}\right) .
$$

As in the inductive step for the upper bound, we rewrite the second factor:

$$
\left(1-4 e^{-\tau|x|}\right)=e^{-4 e^{-\tau|x|}(1+o(1))} .
$$

Then the bound becomes

$$
\left|\tilde{Q}_{j}(w, x)\right| \geq e^{\epsilon(b(p|x|)+b(q|x|))-4 e^{-\tau|x|}(1+o(1))} .
$$

Now, by definition of $b(x), b(p|x|)+b(q|x|)=-a(|x|)+1$. As in the upper bound proof, the second term (i.e., $\epsilon$ ) in the exponent of the right-hand side of (42) after applying this identity is a constant, while the third term decays exponentially, so that the exponent can be lower bounded by $-a(|x|)=b(|x|)$. This concludes the proof.

These lemmas give us enough tools to bound $\left|S_{j}(w, x)\right|$ :

Corollary 2 (Uniform bound on $\left|S_{j}(w, x)\right|$ inside a cone). There exist some $\theta_{0} \in(0, \pi / 2), x_{0}$, and positive constants $C$ and $D$ such that, for sufficiently large $n$ and for $x \in \mathcal{C}(\theta)$ with $|x| \geq x_{0}$ and $|\theta| \leq \theta_{0}$, for any $j \leq k$,

$$
\left|S_{j}(w, x)\right| \leq D|x| e^{-C|x|} .
$$

Moreover, for $|x|<x_{0}$,

$$
\left|S_{j}(w, x)\right| \leq D|w-1||x| e^{-C|x|} .
$$

Proof. This is a combination of Lemmas 12, 13, and 14. We will first show that the claimed inequality holds for sufficiently large $x$. We write, using the triangle inequality,

$$
\left|S_{j}(w, x)\right| \leq \frac{\left|Q_{j}(w, p x)\right|+\left|Q_{j-1}(w, p x)\right|+\left|Q_{j}(w, q x)\right|+\left|Q_{j-1}(w, q x)\right|}{\left|Q_{j-1}(w, p x) Q_{j-1}(w, q x)\right|} .
$$

Note that, by using the triangle inequality on the numerator, we have completely ignored the cancellations in the differences $Q_{j}(w, c x)-Q_{j-1}(w, c x)$. We will rectify this later.

Next, since we are assuming that $x \rightarrow \infty$, we apply Lemma 14 to conclude that the numerator of the above upper bound is equal to

$$
e^{p|x| \cos (\arg (x))(1+o(1))},
$$

while the denominator becomes

$$
e^{p|x| \cos (\arg (x))(1+o(1))+q|x| \cos (\arg (x))(1+o(1))}=e^{|x| \cos (\arg (x))(1+o(1))} .
$$


This gives an asymptotic upper bound of

$$
e^{-q|x| \cos (\arg (x))(1+o(1))},
$$

which can certainly be upper bounded by

$$
|x| e^{-q|x| \cos (\arg (x))(1+o(1))} .
$$

This implies that there is some specific $x_{0}$ such that, provided $|x| \geq x_{0}$,

$$
\left|S_{j}(w, x)\right| \leq|x| e^{-C|x|},
$$

for some positive $C$ (here we have used the fact that $x \in \mathcal{C}(\theta)$, which ensures that $\cos (\arg (x))$ is not too small).

Now, we assume that $|x|<x_{0}$. In this case, instead of applying the triangle inequality to the differences to bound the numerator of $\left|S_{j}(w, x)\right|$, we apply Lemma 13, which gives an upper bound of

$$
O\left(\frac{|x| e^{p|x|}}{g(n)}\right)=O\left(|w-1||x| e^{p|x|}\right)
$$

for the numerator. For the denominator, we apply Lemma 12, which tells us that

$$
Q_{j-1}(w, p x) Q_{j-1}(w, q x) \sim e^{x},
$$

so that

$$
\left|Q_{j-1}(w, p x) Q_{j-1}(w, q x)\right| \sim\left|e^{x}\right|=e^{|x| \cos (\arg (x))} .
$$

Combining these estimates shows that

$$
\left|S_{j}(w, x)\right|=O\left(|w-1||x| e^{p|x|-|x| \cos (\arg (x))}\right),
$$

and, using the fact that $|x|<x_{0}$, so that $e^{|x|(p-\cos (\arg (x)))}=O(1)$,

$$
\left|S_{j}(w, x)\right|=O\left(|w-1||x| e^{-C|x|}\right),
$$

for some positive $C$.

Now, provided that we choose $w-1$ tending to 0 slowly enough with $n$ (i.e., $w-1=n^{o(1)}$ ), instead of evaluating the integrals in the definition of $R_{2, k}(u, z)$ via the residue theorem, we can upper bound them trivially by the product of the maximum of the modulus of the integrand on $\mathcal{C}$ and the length of $\mathcal{C}$. Recalling that $r(n)$ denotes the radius of the circle $\mathcal{C}$, this gives

$$
\left|\oint_{\mathcal{C}} \frac{\log \left(1+S_{j}(w, x)\right)}{(w-1)^{3}(w-u)} \mathrm{d} w\right| \sim\left|\oint_{\mathcal{C}} \frac{S_{j}(w, x)}{(w-1)^{4}} \mathrm{~d} w\right| \leq r(n)^{-O(1)} D|x| e^{-C|x|},
$$

where we've applied Corollary 2 to establish the asymptotic equivalence and the inequality, and we've used the fact that $w-1 \gg u-1$, which implies that $w-u \sim w-1$.

Bounding the terms where $j=o(\log n)$ and $j \sim k$

With this inequality in hand, we can start to bound $R_{2, k}(u, z)$. We first bound the contributions of the ranges $j=o(\log n)$ and $j \sim k$. We start by applying the bound (43) on the absolute value of the integral, which gives, for any $j$, a contribution of

$$
r(n)^{-O(1)} D \sum_{m=0}^{k-j}\left(\begin{array}{c}
k-j \\
m
\end{array}\right) p^{m} q^{k-j-m} n e^{-C p^{m} q^{k-j-m} n}
$$


to the outer sum of $R_{2, k}(u, z)$. Since the factor $r(n)^{-O(1)} D$ is insignificant to the analysis, we focus on bounding the sum. For $j=o(\log n)$, the analysis is essentially the same as that of $R_{1, k}(u, z)$, and we conclude that the sum is $O\left(n^{\beta(\alpha)}\right)$.

In the range where $j \sim k$, we note that $k-j=o(\log n)$. This implies that, for any $m$,

$$
\left(\begin{array}{c}
k-j \\
m
\end{array}\right) \leq e^{\Theta(k-j)}=e^{o(\log n)}=n^{o(1)},
$$

where the inequality follows from the fact that the maximum value of a binomial coefficient $\left(\begin{array}{l}y \\ .\end{array}\right)$ is exponential in $y$. Combining this with the fact that $p^{m} q^{k-j-m} n e^{-C p^{m} q^{k-j-m} n}=O(1)$, we get that the contribution of $j$ in this range is subpolynomial in $n$, so negligible.

Bounding the terms in the middle range: $j, k-j=\Theta(\log n)$

We now turn to the bounding of the middle range, where $j, k-j=\Theta(\log n)$. We define $x_{m, j}=$ $x=p^{m} q^{k-j-m} z$, for any $j$ in the range under consideration, and we split the $m$ sum into two parts: those terms for which $x \gg \log n$, where we can use the coarse upper bound on $S_{j}(w, x)$ which does not take into account cancellation, and those for which $x=O(\log n)$, which requires a more refined estimate of $S_{j}(w, x)$.

Bounding the terms where $x=O(\log n)$

The first task is to more precisely bound $S_{j}(w, x)$ by taking into account the significant cancellations inherent in its definition. Writing $Q_{j}(w, x)$ and $Q_{j-1}(w, x)$ as power series and applying the initial condition $Q_{\ell, j}(u)=1$ for $\ell \leq j$, we have

$$
Q_{j}(w, x)-Q_{j-1}(w, x)=\sum_{\ell \geq j} \mathbb{E}\left[w^{B_{\ell, j}}-w^{B_{\ell, j-1}}\right] \frac{x^{\ell}}{\ell !} .
$$

Since $j=\Theta(\log n)$, we have already identified a significant source of cancellation, but this is not yet sufficient. Recalling that (11) of Theorem 1 gives a superexponentially decaying upper bound on $\mu_{\ell, j}$ for $\ell \leq C j$, for any fixed $C$, we can get a tight bound on the remaining sum. The idea is to determine a bound on $\mathbb{E}\left[w^{B_{\ell, j}}\right]$ by conditioning on the value of $B_{\ell, j}$, then using Markov's inequality and the bound on $\mu_{\ell, j}$ to get a bound on the probabilities and conditional expectations that arise.

In what follows, we first restrict our attention to the initial terms of the sum (44); i.e., we assume that $\ell \leq C j$ for some fixed $C$. Letting $c_{1}, c_{2}$ be the constants in (11) (which we can apply because $j=\Theta(\log n) \rightarrow \infty$ and $\ell=\Theta(j))$, we have the bound

$$
\mu_{\ell, j} \leq c_{1} \ell ! e^{-c_{2} \ell^{2}}
$$

Now, as promised, we compute $\mathbb{E}\left[w^{B_{\ell, j}}\right]$ by conditioning on whether or not $B_{\ell, j} \leq t \mu_{\ell, t}$, for $t$ which we will pick later. This results in

$$
\mathbb{E}\left[w^{B_{\ell, j}}\right]=\mathbb{E}\left[w^{B_{\ell, j}} \mid B_{\ell, j} \leq t \mu_{\ell, j}\right] \operatorname{Pr}\left[B_{\ell, j} \leq t \mu_{\ell, j}\right]+\mathbb{E}\left[w^{B_{\ell, j}} \mid B_{\ell, j}>t \mu_{\ell, j}\right] \operatorname{Pr}\left[B_{\ell, j}>t \mu_{\ell, j}\right] .
$$

Now, we claim that, for an appropriate choice of $t$, the first term is $1+o(1)$, while the second is $o(1)$ (in fact, superexponentially decaying in $\ell$ ). Fix $c_{3} \in\left(0, c_{2}\right)$ and choose $t=e^{c_{3} \ell^{2}}$ (this choice of $t$ is motivated by the requirements that it should not be too small (or lower/upper bounding the first/second probability becomes difficult) or too large (otherwise, upper bounding the second expectation becomes difficult)). Then, by Markov's inequality,

$$
\operatorname{Pr}\left[B_{\ell, j} \leq t \mu_{\ell, j}\right] \geq 1-e^{-c_{3} \ell^{2}}=1-o(1) .
$$


Conditioning on the event that $B_{\ell, j} \leq t \mu_{\ell, j}=e^{-\left(c_{2}-c_{3}\right) \ell^{2}}=o(1)$ and writing $w=1+g(n)$, where $g(n)=o(1)$ (recall that this follows from our choice of integration contour such that $|w-1|=o(1)$ ), gives

$$
w^{B_{\ell, j}}=(1+g(n))^{B_{\ell, j}} \sim 1+g(n) B_{\ell, j}=1+O\left(g(n) e^{-\Theta\left(\ell^{2}\right)}\right) .
$$

Noting that the last expression does not depend on $B_{\ell, j}$, this implies

$$
\mathbb{E}\left[w^{B_{\ell, j}} \mid B_{\ell, j} \leq t \mu_{\ell, j}\right]=1+O\left(g(n) e^{-\Theta\left(\ell^{2}\right)}\right) .
$$

Thus, the first term of $\mathbb{E}\left[w^{B_{\ell, j}}\right]$ (i.e., (45)) is $1+o(1)$, as desired. Turning to the second term, the probability is bounded above by $e^{-\Theta\left(\ell^{2}\right)}$, by Markov's inequality, and we use the a priori upper bound on the expectation; that is, we define $\xi(w)=1+|w-1| \geq 1$, and then the expectation is bounded above by $\xi(w)^{\ell}$ (see the derivation of the inequality (41)). The entire term is then upper bounded by $e^{-\Theta\left(\ell^{2}\right)}$, so that it is negligible with respect to the first. Thus, we have shown that $\mathbb{E}\left[w^{B_{\ell, j}}\right]=1+e^{-\Theta\left(\ell^{2}\right)}$, where the $\Theta$ is uniform in $j$, but dependent on the $C$ for which $\ell \leq C j$. This implies that

$$
\mathbb{E}\left[w^{B_{\ell, j}}-w^{B_{\ell, j-1}}\right]=e^{-\Theta\left(\ell^{2}\right)} .
$$

Applying this estimate to the difference appearing in $S_{j}(w, x)$, we get (suppressing ceiling functions in the indices for convenience)

$$
Q_{j}(w, x)-Q_{j-1}(w, x)=\sum_{\ell=j}^{C j} e^{-\Theta\left(\ell^{2}\right)} \frac{x^{\ell}}{\ell !}+\sum_{\ell>C j} \mathbb{E}\left[w^{B_{\ell, j}}-w^{B_{\ell, j-1}}\right] \frac{x^{\ell}}{\ell !} .
$$

Since $x=O(\log n)$, we can bound the first sum as follows:

$$
\left|\sum_{\ell=j}^{C j} e^{-\Theta\left(\ell^{2}\right)} \frac{x^{\ell}}{\ell !}\right| \leq e^{-\Theta\left((\log n)^{2}\right)+O(\log n \log \log n)}=e^{-\Theta\left((\log n)^{2}\right)},
$$

using the fact that $j=\Theta(\log n)$. Here, the $\ell$ ! in the denominator gives rise to $e^{-\Theta(\log n \log \log n)}$, and the $x^{\ell}$ is upper bounded by $e^{O(\log n \log \log n)}$, where the $\log \log n$ comes from the fact that $x=O(\log n)$, while the $\log n$ results from $\ell \leq C j=\Theta(\log n)$. The second sum can be bounded using the a priori bound (again, see the derivation of the inequality (41)) to get

$$
2 \frac{(\xi(w)|x|)^{C j}}{(C j) !} \cdot e^{\frac{|x|}{C j}}
$$

and, again when $x=O(\log n)$, this becomes

$$
e^{o(\log n \log \log n)-\Theta(\log n \log \log n)}=e^{-\Theta(\log n \log \log n)} .
$$

Thus, overall, we have that

$$
\left|Q_{j}(w, x)-Q_{j-1}(w, x)\right| \leq e^{-\Theta(\log n \log \log n)} .
$$

With these bounds in hand, we are ready to handle the relevant terms of $R_{2, k}(u, z)$. Our refined upper bound on $\left|Q_{j}(w, x)-Q_{j-1}(w, x)\right|$ implies

$$
\left|S_{j}(w, x)\right| \leq \frac{e^{-\Theta(\log n \log \log n)}}{\left|Q_{j-1}(w, p x) Q_{j-1}(w, q x)\right|},
$$


and, since $Q_{j-1}(w, c x) \sim e^{c x}$, and $|x|$ is at least $e^{-O(\log n)}$, this shows that

$$
\left|S_{j}(w, x)\right| \leq e^{-\Theta(\log n \log \log n)} .
$$

Then

$$
\begin{aligned}
\left(\begin{array}{c}
k-j \\
m
\end{array}\right)\left|\oint_{\mathcal{C}} \frac{\log \left(1+S_{j}(w, x)\right)}{(w-1)^{4}} \mathrm{~d} w\right| & \leq r(n)^{-O(1)} e^{O(\log n)-\Theta(\log n \log \log n)} \\
& =r(n)^{-O(1)} e^{-\Theta(\log n \log \log n)} \\
& =o(1) .
\end{aligned}
$$

Here, $r(n)^{-O(1)}$ comes from $(w-1)^{4}$ in the denominator of the integrand. The $e^{O(\log n)}$ is a result of upper bounding the binomial coefficient, since $k-j=O(\log n)$ and the binomial coefficient is at most exponential in its top number. Finally, the $e^{-\Theta(\log n \log \log n)}$ comes from the bound on $\left|S_{j}(w, x)\right|$ and the Taylor expansion for the log.

Bounding the terms where $x \gg \log n$

For $x \gg \log n$, using the coarse upper bound on $\left|S_{j}(w, x)\right|$ gives

$$
\begin{aligned}
(1+o(1))\left(\begin{array}{c}
k-j \\
m
\end{array}\right)\left|\oint_{\mathcal{C}} \frac{\log \left(1+S_{j}(w, x)\right)}{(w-1)^{4}} \mathrm{~d} w\right| & \leq\left(\begin{array}{c}
k-j \\
m
\end{array}\right) r(n)^{-O(1)} e^{-\Theta(x)} \\
& \leq r(n)^{-O(1)} e^{O(\log n)-\Theta(x)} \\
& =r(n)^{-O(1)} e^{-\Theta(x)}=o(1)
\end{aligned}
$$

so that these terms are negligible. Note that the $e^{O(\log n)}$ comes from upper bounding the binomial coefficient: it is at most $e^{\Theta(k)}=e^{\Theta(\log n)}$.

Since the contributions of all ranges have been shown to be either negligible or $O\left(n^{\beta(\alpha)}\right)$, this completes the proof that $R_{2, k}(u, z)=O\left(n^{\beta(\alpha)}\right)$. Having shown the remainder term $\frac{1}{3 !} R[\tilde{l}]_{k}(u, z)$ to be negligible, we have thus established a central limit theorem for the (normalized) external profile in the Poisson model.

We complete the proof by a bare-hands de-Poissonization to recover a central limit theorem for the Bernoulli model. We relegate the details to Appendix 4.3.3.

\section{Appendix}

\subsection{Well definedness and analyticity of series related to $A_{k}(s)$}

\section{Proof of Lemma 2. Proof of (i)}

Without loss of generality, we can assume that $g(n)=C n$, for some positive constant $C$, because the assumption $g(n)=\Omega(n)$ implies that, for large enough $n, g(n) \geq C n$. Next, we apply the ratio test, which gives

$$
\left|e^{-(g(n+1))-g(n))} \frac{\phi_{n+1}(s) n !}{(n+1) ! \phi_{n}(s)}\right|=\left|\frac{n+s}{n+1}\right| e^{-(g(n+1)-g(n))} \sim e^{-(g(n+1)-g(n))} .
$$

Now, using the assumption about the growth of $g(n)$, we have that the ratio is asymptotically less than 1 , so that the series converges absolutely.

Proof of (ii)

If we can show that $F_{j}(s)$ is analytic at $s=0$, then the result follows, since a function defined by a 
power series at a given point is analytic at all points inside its disc of convergence. We'll start by looking at $\left[s^{m}\right] \phi_{n}(s)$. Toward that end, define $S_{m}$ to be the set of all subsets of size $n-1-m$ of the set $[n-1]$. Then, noting that $\phi_{n}(s)$ is a product of $n-1$ monomials, so that each choice of $m$ such monomials gives a contribution to $\left[s^{m}\right] \phi_{n}(s)$, we have

$$
\left[s^{m}\right] \phi_{n}(s)=\sum_{X \in S_{m}} \prod_{x \in X} x .
$$

Since $\left|S_{m}\right|=\left(\begin{array}{c}n-1 \\ n-1-m\end{array}\right)=\left(\begin{array}{c}n-1 \\ m\end{array}\right)$ and $\prod_{x \in X} x \leq \frac{(n-1) !}{m !}$,

$$
\left[s^{m}\right] \phi_{n}(s) \leq \frac{(n-1) !}{m !(n-1-m) !} \cdot \frac{(n-1) !}{m !}=\frac{((n-1) !)^{2}}{m !^{2}(n-1-m) !} .
$$

Now,

$$
\left[s^{m}\right] F_{j}(s)=\sum_{n \geq j} e^{-\Omega(n)}\left[s^{m}\right] \phi_{n}(s) \frac{1}{n !}
$$

and

$$
\begin{aligned}
& \left|e^{-\Omega(n)}\left[s^{m}\right] \phi_{n}(s) \frac{1}{n !}\right| \leq e^{-\Omega(n)} \frac{1}{n !} \frac{(n-1) !^{2}}{m !^{2}(n-1-m) !} \\
& =e^{-\Omega(n)} \frac{(n-1) !}{n m !^{2}(n-1-m) !} \stackrel{n \rightarrow \infty}{\sim} \frac{e^{-\Omega(n)} n^{m-1}}{m !^{2}} .
\end{aligned}
$$

The series with these terms converges because of the exponential decay of $e^{-\Omega(n)}$ as $n \rightarrow \infty$. This implies that the series defining $\left[s^{m}\right] F_{j}(s)$ converges, so that $F_{j}(s)$ is analytic at 0.

\subsection{Rough upper bounds on $\mu_{n, k}, c_{n, k}, \tilde{G}_{k}(z), \tilde{V}_{k}(z)$}

\subsubsection{Upper bounds on $\left|\tilde{G}_{k}(z)\right|$ as $z \rightarrow \infty$}

\section{Proof of Lemma 1. Proof of (i)}

We proceed by induction on $k$, then on increasing domains.

\section{Base case for induction on $k$}

For $k=0$, we have $\tilde{G}_{0}(z)=z e^{-z}$, so that

$$
\left|\tilde{G}_{0}(z)\right|=|z|\left|e^{-z}\right|=|z| e^{-\Re(z)},
$$

which, for large enough $|z|$ in the cone, is less than $C|z|^{1+\epsilon}$ (and, in fact, any $C|z|$ ), for any choice of $C>0$.

\section{Inductive step for $k$}

We now assume that the claimed bound is true for $0 \leq j<k$, and we prove the claim for $k$ via induction on increasing domains.

Increasing domains base case

For the base case, observe that an upper bound on $\left|\tilde{G}_{k}(z)\right|$ that is uniform in $k$ holds:

$$
\begin{aligned}
\tilde{G}_{k}(z) & =e^{-z} \sum_{n \geq k+1} \mu_{n, k} \frac{z^{n}}{n !} \\
& =z e^{-z} \sum_{n \geq k+1} \mu_{n, k} \frac{z^{n-1}}{n !} \\
& \Longrightarrow\left|\tilde{G}_{k}(z)\right| \leq|z| e^{|z|-\Re(z)},
\end{aligned}
$$


for any $z$, where we've used the fact that $\mu_{n, k} \leq n$. In particular, this applies in the truncated cone $\mathcal{C}(\theta, R)$. Since $\mathcal{C}(\theta, R)$ is compact, and both the upper bound on $\left|\tilde{G}_{k}(z)\right|$ and $|z|^{1+\epsilon}$ are continuous, it has a maximum value in $\mathcal{C}(\theta, R)$, so that there is some $C=C(R)$ for which

$$
\left|\tilde{G}_{k}(z)\right| \leq C|z|^{1+\epsilon}
$$

which establishes the base case.

Inductive step for increasing domains induction

For the inductive step, we start with the recurrence for $\tilde{G}_{k}(z)$ :

$$
\begin{aligned}
\left|\tilde{G}_{k}(z)\right| & \leq\left|e^{-q z}\right|\left|\tilde{G}_{k}(z p)\right|+\left|e^{-p z}\right|\left|\tilde{G}_{k}(z q)\right|+\left|\tilde{G}_{k-1}(p z)\right|\left|1-e^{-q z}\right|+\left|\tilde{G}_{k-1}(q z)\right|\left|1-e^{-p z}\right| \\
& \leq\left(\left|e^{-q z}\right|+\left|1-e^{-q z}\right|\right) C p^{1+\epsilon}|z|^{1+\epsilon}+\left(\left|e^{-p z}\right|+\left|1-e^{-p z}\right|\right) C q^{1+\epsilon}|z|^{1+\epsilon}
\end{aligned}
$$

Now, for any positive $c$,

$$
\left|e^{-c z}\right|+\left|1-e^{-c z}\right| \stackrel{z \rightarrow \infty}{\longrightarrow} 1
$$

with $z$ in the cone. Thus, we can choose $|z|$ large enough (depending on $\epsilon$ ) so that, simultaneously,

$$
\left(\left|e^{-q z}\right|+\left|1-e^{-q z}\right|\right) p^{1+\epsilon}<p
$$

and

$$
\left(\left|e^{-p z}\right|+\left|1-e^{-p z}\right|\right) q^{1+\epsilon}<q,
$$

which gives

$$
\left|\tilde{G}_{k}(z)\right| \leq C p|z|^{1+\epsilon}+C q|z|^{1+\epsilon} \leq C|z|^{1+\epsilon}
$$

\section{Proof of (ii)}

Recall the functional equation for $\tilde{G}_{j}(z)$.

$$
\tilde{G}_{j}(z)=L[\tilde{G}]_{j-1}(z)+T[\tilde{G}]_{j}(z) .
$$

Iterating this recurrence, we get

$$
\tilde{G}_{j}(z)=L^{j}[\tilde{G}]_{0}(z)+\sum_{\ell=0}^{j-1} L^{\ell} T[\tilde{G}]_{j-\ell}(z),
$$

where, recall, $\tilde{G}_{0}(x)=x e^{-x}$. Applying the definition of $L$, the first term becomes

$$
\sum_{\ell=0}^{j}\left(\begin{array}{l}
j \\
\ell
\end{array}\right) \tilde{G}_{0}\left(p^{\ell} q^{j-\ell} z\right)=\sum_{\ell=0}^{j}\left(\begin{array}{l}
j \\
\ell
\end{array}\right) p^{\ell} q^{j-\ell} z e^{-p^{\ell} q^{j-\ell} z} .
$$

Now, for positive real $z$, we have

$$
p^{\ell} q^{j-\ell} z \geq q^{j} z
$$

so that

$$
e^{-p^{\ell} q^{j-\ell} z} \leq e^{-q^{j} z} .
$$

Taking the absolute value of the first sum, applying the triangle inequality and the observation just made, and pulling the resulting exponential factor and $|z|$ out of the summation gives an upper bound of

$$
|z| e^{-q^{j}|z| \cos (\arg (z))} \sum_{\ell=0}^{j}\left(\begin{array}{l}
j \\
\ell
\end{array}\right) p^{\ell} q^{j-\ell}=|z| e^{-q^{j}|z| \cos (\arg (z))} .
$$


Turning to the second summation, we consider the $\ell$ th term:

$$
L^{\ell} T[\tilde{G}]_{j-\ell}(z)=\sum_{r=0}^{\ell}\left(\begin{array}{l}
\ell \\
r
\end{array}\right) T[\tilde{G}]_{j-\ell}\left(p^{r} q^{\ell-r} z\right) .
$$

Now, we upper bound $\tilde{G}_{j-\ell}(x)$ and $\tilde{G}_{j-\ell-1}(x)$ by $C^{\prime}|x|^{1+\epsilon}$, for some $C^{\prime}$ independent of $C$, and an arbitrarily small $\epsilon>0$. This we can do by part (i). This implies that

$$
\begin{aligned}
\left|T[\tilde{G}]_{j-\ell}(x)\right| & =\left|e^{-p x}\left(\tilde{G}_{j-\ell}(q x)-\tilde{G}_{j-\ell-1}(q x)\right)+e^{-q x}\left(\tilde{G}_{j-\ell}(p x)-\tilde{G}_{j-\ell-1}(p x)\right)\right| \\
& \leq 4 C^{\prime} p e^{-q|x| \cos (\arg (x))}|x|^{1+\epsilon} .
\end{aligned}
$$

Plugging this into (53) gives an upper bound of

$$
4 C^{\prime} p \sum_{r=0}^{\ell}\left(\begin{array}{l}
\ell \\
r
\end{array}\right) p^{r} q^{\ell-r}|z|^{1+\epsilon} e^{-p^{r} q^{\ell-r+1}|z| \cos (\arg (z))} \leq 4 C^{\prime} p|z|^{1+\epsilon} e^{-q^{\ell+1}|z| \cos (\arg (z))} .
$$

Here, we've used the fact that $p^{r} q^{-r}=(p / q)^{r} \geq 1^{r}=1$ to upper bound the exponent. After this, the only factor of the above expression which contain $r$ is

$$
\sum_{r=0}^{\ell}\left(\begin{array}{l}
\ell \\
r
\end{array}\right) p^{r} q^{\ell-r}=1
$$

The resulting upper bound is maximized when $\ell=j-1$, so that the second sum of (52) is upper bounded by

$$
4 C^{\prime} p|z|^{1+\epsilon} j e^{-q^{j}|z| \cos (\arg (z))} .
$$

Thus, we have an upper bound of

$$
\left|\tilde{G}_{j}(z)\right| \leq|z|^{1+\epsilon} e^{-q^{j}|z| \cos (\theta)}\left(1+4 C^{\prime} p j\right) .
$$

Now, since $j \leq C$, we finally have

$$
\left|\tilde{G}_{j}(z)\right| \leq|z|^{1+\epsilon} e^{-q^{C}|z| \cos (\theta)}\left(1+4 C C^{\prime} p\right) .
$$

\subsubsection{Superexponentially decaying bound on $\mu_{n, k}$ for $k=\Theta(n)$}

We now aim to prove (11) of Theorem 1. The natural way to do this is by induction on $m$ and using the recurrence for $\mu_{m, j}$, but the inductive hypothesis cannot then be applied for all $h<m$ : there appear terms of the form $\mu_{h, j-1}$ in the recurrence, and it is sometimes the case that

$$
C h>(j-1),
$$

which happens precisely when $h>m-1 / C$. Thus, we must first prove a similar lemma which bounds $\mu_{m, j}$ whenever $m-j<\ell$, for any fixed $\ell \geq 0$.

Lemma 15. For any $C>1$, there exist $c_{1}, c_{2}>0$ such that, for $n$ large enough,

$$
\mu_{n, m} \leq c_{1} n ! e^{-c_{2} m^{2}}
$$

whenever $m \geq n-C$. 
Proof. This is by induction on $n$.

\section{Base case}

For the base case, we show that, for any $M \geq 0$, we can find $c_{1}$ and $c_{2}$ such that the claimed inequality is satisfied whenever $n \leq M$. Given any $M \geq 0$ and $c_{2}>0$, we have, for $n \leq M$,

$$
\mu_{n, m} \leq M,
$$

and, provided that we take

$$
c_{1} \geq \frac{M}{M !} e^{c_{2} M^{2}}
$$

this implies that

$$
\mu_{n, m} \leq c_{1} n ! e^{-c_{2} m^{2}}
$$

for all $n, m \leq M$.

\section{Inductive step}

For the inductive step, we assume that, for appropriately chosen $c_{1}, c_{2}$, the claimed inequality holds for $\mu_{n^{\prime}, m^{\prime}}$ for any $n^{\prime}<n$ with $n>M$, and for any $m^{\prime}$. In what follows, we will derive a condition on $c_{2}$ which must (and can) be satisfied in order for the induction to work. Now, by the recurrence for $\mu_{n, m}$ and the fact that $m \geq n-C$,

$$
(1-T(-n)) \mu_{n, m}=\sum_{j=n-C}^{n-1}\left(\begin{array}{l}
n \\
j
\end{array}\right) p^{j} q^{n-j} \mu_{j, m-1},
$$

and, since $j \leq n-1$, we have

$$
m-1 \geq(n-1)-C \geq j-C,
$$

so that the inductive hypothesis can be applied to each term in the sum:

$$
(1-T(-n)) \mu_{n, m} \leq \sum_{j=n-C}^{n-1}\left(\begin{array}{l}
n \\
j
\end{array}\right) p^{n} \cdot c_{1} j ! e^{-c_{2}(m-1)^{2}}=c_{1} n ! p^{n} \sum_{j=n-C}^{n-1} \frac{e^{-c_{2}(m-1)^{2}}}{(n-j) !}
$$

We can further upper bound this by

$$
c_{1} C n ! p^{n} e^{-c_{2}(m-1)^{2}}
$$

and our goal is now to choose $c_{2}$ such that

$$
C p^{n} e^{2 c_{2} m-c_{2}} /(1-T(-n)) \leq 1-\epsilon,
$$

for some positive constant $\epsilon$. We need

$$
e^{c_{2}(2 m-1)} \leq \frac{(1-\epsilon)(1-T(-n))}{C p^{n}} .
$$

Taking logarithms and dividing both sides by $2 m-1$, we must have, equivalently,

$$
c_{2} \leq \frac{\log (1-\epsilon)-\log C+n \log (1 / p)+\log (1-T(-n))}{2 m-1} .
$$

The required upper bound is lower bounded by

$$
\frac{\log (1-\epsilon)-\log C+n \log (1 / p)+\log (1-T(-n))}{2 n-1},
$$


which tends to $\log (1 / p) / 2$ as $n \rightarrow \infty$. Thus, provided $n$ is sufficiently large (depending only on $C$ and $\epsilon$; this can be enforced by choosing a sufficiently large $M$ ), the required upper bound is clearly positive, so that a $c_{2}$ which satisfies it can be chosen. Then $c_{1}$ can be chosen as dictated by the base case, and we have

$$
\mu_{n, m} \leq c_{1} n ! e^{-c_{2} m^{2}}
$$

as desired.

We can now prove (11).

Proof of (11). Throughout, we suppress floor and ceiling functions, which are insignificant to the analysis.

The proof is again by induction on $n$.

\section{Base case}

By the same argument as in the proof of Lemma 15, for any $M$ and $c_{2}, c_{1}$ can be chosen appropriately so as to ensure that the claimed inequality holds for $n, m \leq M$.

\section{Inductive step}

We now proceed with the induction. Again using the recurrence and the fact that $m \geq C n$, we have

$$
(1-T(-n)) \mu_{n, m} \leq \sum_{j=C n}^{n-1}\left(\begin{array}{l}
n \\
j
\end{array}\right) p^{n}\left(\mu_{j, m-1}+\mu_{n-j, m-1}\right) .
$$

Now, we only know that $m-1 \geq C n-1=C(n-1 / C)$. That is, for some of the terms in the sum, we cannot apply the induction hypothesis. To circumvent this problem, we split the sum into two parts, one of which we handle by the induction hypothesis, and the other by Lemma 15. That is, we will upper bound by

$$
\sum_{j=C n}^{n-1 / C}\left(\begin{array}{l}
n \\
j
\end{array}\right) p^{n}\left(\mu_{j, m-1}+\mu_{n-j, m-1}\right)+\sum_{j=n-1 / C+1}^{n-1}\left(\begin{array}{l}
n \\
j
\end{array}\right) p^{n}\left(\mu_{j, m-1}+\mu_{n-j, m-1}\right) .
$$

We now upper bound the first sum: applying the induction hypothesis, we can upper bound it by

$$
n ! p^{n} c_{1} e^{-c_{2}(m-1)^{2}} \sum_{j=C n}^{n-1 / C}\left[\frac{1}{(n-j) !}+\frac{1}{j !}\right] \leq c_{1} n ! p^{n} e^{-c_{2}(m-1)^{2}}\left[\frac{1}{(1 / C) !}+\frac{1}{(C n) !}\right] n(1-C) .
$$

We thus require that

$$
\operatorname{Dnp}^{n} e^{c_{2}(2 m-1)} /(1-T(-n)) \leq 1-\epsilon,
$$

where $D$ is some positive constant, and $\epsilon$ is any positive constant less than 1 . Just as in the proof of Lemma 15, we can choose $c_{2}$ small enough so that this holds for any $n$ sufficiently large.

The second sum is handled analogously, and we choose the minimum of the two resulting constants for $c_{2}$. We then choose $c_{1}$ sufficiently large, and this completes the proof.

\subsubsection{Bounds on $\left|\tilde{C}_{k}(z)\right|$ and $\left|\tilde{V}_{k}(z)\right|$ as $z \rightarrow \infty$}

\section{Proof of Lemma 10. Proof of (i)}

We will prove a slightly stronger claim, because it will help in the implementation of the induction. In particular, we claim that the inequality holds for any $z$ in the cone, regardless of magnitude. 
To establish the claim for $z$ in a compact region of the cone including the origin, we prove the following: the upper bound (uniform in $k$ ) of

$$
\left|\tilde{C}_{k}(z)\right| \leq|z|^{2} e^{|z|-\Re(z)}
$$

which holds for any $z \in \mathbb{C}$, shows that there is some positive constant $C$ for which the inequality holds for any $k$ whenever $|z| \leq R$. The proof is as follows:

$$
\begin{aligned}
\left|e^{-z}\right|\left|\sum_{m \geq 0} c_{m, k} \frac{z^{m}}{m !}\right| & \leq e^{-|z| \cos (\arg (z))} \sum_{m \geq 0}\left|c_{m, k}\right| \frac{|z|^{m}}{m !} \\
& \leq e^{-|z| \cos (\arg (z))} \sum_{m \geq 0} m(m-1) \frac{|z|^{m}}{m !} \\
& \leq|z|^{2} e^{|z|-|z| \cos (\arg (z))},
\end{aligned}
$$

where we've used the fact that $c_{m, k} \leq m(m-1)$, itself a consequence of the bound

$$
B_{m, k} \leq m
$$

which holds for all $m$. The remaining task is to demonstrate the polynomial upper bound for $|z|>R$.

\section{Base case}

For the base case, $C_{0}(z)=0$, and the inequality is trivially true throughout the cone.

\section{Inductive step}

We now assume that the claimed inequality holds for $k-1$, and we demonstrate it for $k$. We have, by the recurrence for $\tilde{C}_{k}(z)$ and the inductive hypothesis,

$$
\left|\tilde{C}_{k}(z)\right| \leq\left(\left|e^{-q z}+\right| 1-e^{-q z} \mid\right) C p^{2+\epsilon}|z|^{2+\epsilon}+\left(\left|e^{-p z}\right|+\left|1-e^{-p z}\right|\right) C q^{2+\epsilon}|z|^{2+\epsilon}+C_{2} p^{2+\epsilon}|z|^{2+\epsilon}
$$

where $C_{2}>0$ and we've used the fact that we can make $R$ large enough so that

$$
\left|\tilde{G}_{k-1}(z)\right| \leq C_{3}|z|^{1+\epsilon / 2}
$$

for some constant $C_{3}$, by Lemma 1 .

Provided that we choose $C$ large enough, we have $C_{2} p^{2+\epsilon} \leq \epsilon^{\prime} C$, for any positive $\epsilon^{\prime}$. The rest of the proof is as in the expected value case, so we omit it.

\section{Proof of (ii)}

This follows from an easy modification of the proof of Lemma 1, part (ii), so we only sketch the proof.

We note that, as a result of part (i), which gives a polynomial upper bound (in $|z|)$ on the growth of $\left|\tilde{C}_{\ell}(z)\right|$ for all $\ell \leq C$, and Lemma 1, part (ii), we can write, for some constants $C^{\prime}, C^{\prime \prime}>0$,

$$
\tilde{V}_{j}(z)=L[\tilde{V}]_{j-1}(z)+C^{\prime} e^{-C^{\prime \prime}|z|},
$$

and iterating the recurrence shows that $\tilde{V}_{j}(z)$ is a sum of terms which are exponentially decaying in $|z|$. 


\subsubsection{Superexponentially decaying bound on $c_{n, k}$ for $k=\Theta(n)$}

Here we aim to prove (16) of Theorem 2. We start with the recurrence

$$
\begin{aligned}
c_{n, k}(1-T(-n)) & =\sum_{j=k}^{n-1}\left(\begin{array}{c}
n \\
j
\end{array}\right) p^{j} q^{n-j}\left(c_{j, k-1}+c_{n-j, k-1}+2 \mu_{j, k-1} \mu_{n-j, k-1}\right) \\
& \leq \sum_{j=k}^{n-1}\left(\begin{array}{c}
n \\
j
\end{array}\right) p^{j} q^{n-j}\left(c_{j, k-1}+c_{n-j, k-1}\right)+n \sum_{j=k}^{n-1}\left(\begin{array}{c}
n \\
j
\end{array}\right) p^{j} q^{n-j}\left(\mu_{j, k-1}+\mu_{n-j, k-1}\right. \\
& =\sum_{j=k}^{n-1}\left(\begin{array}{c}
n \\
j
\end{array}\right) c_{j, k-1}\left(p^{j} q^{n-j}+p^{n-j} q^{j}\right)+n \mu_{n, k}(1-T(-n)),
\end{aligned}
$$

so that

$$
c_{n, k} \leq \frac{2 p^{n} \sum_{j=k}^{n-1}\left(\begin{array}{c}
n \\
j
\end{array}\right) c_{j, k-1}}{1-T(-n)}+n \mu_{n, k} .
$$

When $n \geq 2$, we can upper bound $T(-n)$ by

$$
T(-n) \leq 2 p^{-n} \leq 2 p^{-2} \leq 2^{1-2}=1 / 2,
$$

so that

$$
\frac{1}{1-T(-n)} \leq 2
$$

First, we will need a simpler lemma.

Lemma 16. For all fixed $\ell \in \mathbb{Z}^{\geq 0}$, there exist positive constants $C_{1}, C_{2}$ such that, for all $n$ and $k \geq n-\ell$,

$$
c_{n, k} \leq C_{1} n ! e^{-C_{2} k^{2}} .
$$

To prove this, we need another bound on $\mu_{n, k}$.

Lemma 17. There exist positive constants $C_{1}^{*}, C_{2}^{*}$ such that, for all fixed $\ell \in \mathbb{Z} \geq 0$, all $n$, and $k \geq n-\ell$,

$$
n \mu_{n, k} \leq C_{1}^{*} n ! e^{-C_{2}^{*} k^{2}} .
$$

Proof. This is an easy consequence of (11).

Proof of Lemma 16. The proof is by induction on $n$.

\section{Base case}

By the initial conditions, $c_{n, k} \leq n^{2}$ for any $n, k$. Thus, fixing some particular $n_{*}$ and considering $k<n_{*}$, we can fix a sufficiently large $C_{1}$ and a $C_{2}>0$ for which the claimed inequality holds for $c_{n^{\prime}, k}, n^{\prime} \leq n_{*}$.

\section{Induction}

Here we assume that the claim is true for $n^{\prime}<n$, with $n \geq n_{*}$. We have

$$
\begin{aligned}
c_{n, k} & \leq 4 p^{n} \sum_{j=k}^{n-1} \frac{n !}{j !(n-j) !} C_{1} j ! e^{-C_{2}(k-1)^{2}}+n \mu_{n, k} \\
& \leq 4 p^{n} \sum_{j=k}^{n-1} \frac{n !}{(n-j) !} C_{1} e^{-C_{2}(k-1)^{2}}+n \mu_{n, k} .
\end{aligned}
$$


We were able to apply the induction hypothesis because $j-(k-1) \leq(n-1)-(k-1) \leq \ell$ for all $j$ over which the sum is taken.

To handle $n \mu_{n, k}$, we apply Lemma 17 . Now,

$$
-C_{2}(k-1)^{2}=-C_{2} k^{2}+2 C_{2} k-C_{2},
$$

so we require that

$$
\frac{4 p^{n}}{(n-k) !} e^{2 C_{2} k-C_{2}}+\frac{1}{2} \leq 1 .
$$

It is easy to see that $C_{2}$ can be chosen to satisfy this inequality for all $n, k \geq n-\ell$ :

$$
e^{C_{2}(2 k-1)} \leq \frac{(n-k) !}{8 p^{n}} \Longleftrightarrow C_{2} \leq \frac{n \log \frac{1}{p}-\log 8+\log ((n-k) !)}{2 k-1} .
$$

The first term of the numerator and the denominator are both $\Theta(n)$ as $n \rightarrow \infty$, while the rest are bounded above and below by constants, so that, at least asymptotically (i.e., provided we've chosen $n_{*}$ large enough), they are the only two that matter. It is thus sufficient to have

$$
C_{2} \leq \frac{1}{2} \log (1 / p)>0 .
$$

Furthermore, if we choose $C_{1}>2 C_{1}^{*}$, we have the claimed inequality.

Now we begin the proof of (16) of Theorem 2 .

Proof of (16) of Theorem 2. The proof is similar to that of the lemma. It is by induction on $n$.

\section{Base case}

The base case is exactly as in the proof of Lemma 16 .

\section{Inductive step}

We now assume that the claim is true for $n^{\prime}<n$, with $n \geq n_{*} \geq 2$, with $n_{*}$ as in the proof of Lemma 16. Let $k \geq C n$. Then, by the inequality (57),

$$
c_{n, k} \leq 4 p^{n} \sum_{j=k}^{n-1}\left(\begin{array}{l}
n \\
j
\end{array}\right) c_{j, k-1}+n \mu_{n, k} .
$$

To upper bound the terms of the sum, we note that we can apply the inductive hypothesis for any $j$ such that $k-1 \geq C j$. Since $k \geq C n$, this means that any $j$ satisfying $j \leq n-1 / C$ is amenable to this approach. This gives, for such $j$,

$$
c_{j, k-1} \leq C_{1} j ! e^{-C_{2}(k-1)^{2}} .
$$

For $j \in\{n-1 / C, \ldots, n-1\}$, we apply Lemma 16 to conclude that there exist $C_{1}^{*}$ and $C_{2}^{*}$ such that

$$
c_{j, k-1} \leq C_{1}^{*} j ! e^{-C_{2}^{*}(k-1)^{2}} .
$$

Provided that we choose $C_{1} \geq C_{1}^{*}$ and $C_{2} \leq C_{2}^{*}$, we can replace $C_{1}^{*}$ and $C_{2}^{*}$ by $C_{1}$ and $C_{2}$ in the above, so that the first sum is upper bounded by

$$
4 p^{n} \sum_{j=k}^{n-1}\left(\begin{array}{l}
n \\
j
\end{array}\right) C_{1} j ! e^{-C_{2}(k-1)^{2}}
$$

Next, to upper bound $n \mu_{n, k}$, we appeal to the superexponentially decaying bound (11), and the rest of the proof proceeds as in that of Lemma 16. 


\subsection{De-Poissonization}

\subsubsection{De-Poissonization of $\tilde{G}_{k}(z)$}

Here we recover the asymptotics of $\mu_{n, k}$ from $\tilde{G}_{k}(z)$. For this, we apply Theorem 1 of [10]. The inner condition follows immediately from Lemma 1 . The outer condition we capture in the following claim.

Lemma 18. Let $\theta \in(0, \pi / 2)$. Then there exist some $\phi<1$ and $C>0$ such that, for $z$ outside $\mathcal{C}(\theta)$ and any $k \geq 0$,

$$
\left|\tilde{G}_{k}(z) e^{z}\right| \leq C e^{\phi|z|} .
$$

Proof. We start by recalling the uniform upper bound on $\left|\tilde{G}_{k}(z)\right|$ : for any $k \geq 0$ and $z \in \mathbb{C}$,

$$
\left|\tilde{G}_{k}(z)\right| \leq|z| e^{|z|-\Re(z)} .
$$

This implies that, for any fixed $R>0$, we can choose a $C>0$ such that the claimed inequality holds whenever $|z| \leq R$, for every $k \geq 0$. It thus remains to check that it holds for $|z|>R, z \notin \mathcal{C}(\theta)$. This we do by induction on $k$.

\section{Base case}

For $k=0, e^{z} \tilde{G}_{0}(z)=z$, and, for any positive $\phi$, an appropriate $R$ can be chosen such that the claimed inequality holds for $|z|>R, z \notin \mathcal{C}(\theta)$. More specifically, given $\phi$, we choose $R$ large enough so that

$$
|z| \leq e^{\phi|z|}
$$

whenever $|z|>R$. Next, we choose $C>1$ and such that $|z| e^{|z|-\Re(z)} \leq C e^{\phi|z|}$ for $|z| \leq R$. This implies that $\left|e^{z} \tilde{G}_{0}(z)\right| \leq C e^{\phi|z|}$ for any $|z|$, as required.

\section{Inductive step}

Now, assuming that the claimed inequality is true for $0 \leq j<k$, we demonstrate that it holds for $k$. In fact, since the recurrence for $\tilde{G}_{k}(z)$ can be put in the form

$$
\tilde{G}_{k}(z)=\gamma_{1}(z) \tilde{G}_{k}(p z)+\gamma_{2}(z) \tilde{G}_{k}(q z)+t(z)
$$

with

$$
\gamma_{1}(z)=e^{-q z}, \quad \gamma_{2}(z)=e^{-p z}, \quad t(z)=\tilde{G}_{k-1}(p z)\left(1-e^{-q z}\right)+\tilde{G}_{k-1}(q z)\left(1-e^{-p z}\right),
$$

it is sufficient to check the outer conditions required by Theorem 10 of [10]: in particular, we need to show that, for $|z|$ sufficiently large and some $\phi<1$,

$$
\left|\gamma_{1}(z)\right| e^{q \Re(z)} \leq \frac{1}{3} e^{\phi q|z|}, \quad\left|\gamma_{2}(z)\right| e^{p \Re(z)} \leq \frac{1}{3} e^{\phi p|z|}, \quad|t(z)| e^{\Re(z)} \leq \frac{1}{3} e^{\phi|z|} .
$$

The first two inequalities easily hold: for $c \in\{p, q\}$,

$$
\left|e^{-c z}\right| e^{c \Re(z)}=e^{-c \Re(z)+c \Re(z)}=1,
$$

and the claimed inequalities hold for any positive $\phi$ and sufficiently large $z$ (in particular, any $|z| \geq \frac{\log 3}{q \phi}$ suffices).

For the third inequality, we apply the induction hypothesis:

$$
|t(z)| e^{\Re(z)} \leq C e^{\phi p|z|}\left|e^{q z}-1\right|+C e^{\phi q|z|}\left|e^{p z}-1\right| .
$$


Choosing $\phi=\cos (\theta)+\epsilon$, for any positive constant $\epsilon$, we have, for any positive $c$,

$$
\left|e^{c z}\right|=e^{c \Re(z)}=e^{c|z| \cos (\arg (z))} \leq e^{c|z|(\phi-\epsilon)},
$$

since $z \notin \mathcal{C}(\theta)$. This implies that

$$
|t(z)| e^{\Re(z)} \leq C\left[e^{\phi p|z|+\phi q|z|-q|z| \epsilon}+e^{\phi q|z|+\phi p|z|-p|z| \epsilon}+e^{\phi p|z|}+e^{\phi q|z|}\right]=C e^{(\phi-q \epsilon)|z|}(1+o(1)),
$$

so that, for sufficiently large $|z|$ (depending only on $\phi, p$ ),

$$
|t(z)| e^{\Re(z)} \leq \frac{1}{3} e^{\phi|z|}
$$

which completes the proof.

\subsubsection{De-Poissonization of variance}

We now de-Poissonize using the following theorem from [10] (rephrased in our notation and simplified):

Theorem 8 (De-Poissonization of variance). Suppose that there is some $\theta \in(0, \pi / 2)$ such that the following conditions hold:

- There is some $\phi \in(0,1)$ such that, for $z$ outside the cone $\mathcal{C}(\theta), e^{z} \tilde{G}_{k}(z)$ and $e^{z}\left(\tilde{V}_{k}(z)+\tilde{G}_{k}(z)^{2}\right)$ are both $O\left(e^{\phi|z|}\right)$.

- There is some $\beta \leq 1$ such that, for $z$ inside $\mathcal{C}(\theta), \tilde{G}_{k}(z)$ and $\tilde{V}_{k}(z)$ are both $O\left(z^{\beta}\right)$.

Then

$$
V_{n, k}=\tilde{V}_{k}(n)-n\left[\tilde{G}_{k}^{\prime}(n)\right]^{2}+O\left(\max \left\{n^{\beta-1}, n^{2 \beta-2}\right\}\right) .
$$

Next we check that the hypotheses of this theorem are satisfied.

Conditions on $\tilde{G}_{k}(z)$

The inner and outer conditions on $\tilde{G}_{k}(z)$ were already verified in the de-Poissonization in the expected value case.

Outer condition on $\tilde{V}_{k}(z)+\tilde{G}_{k}(z)^{2}$

We now demonstrate that the outer condition holds for $\tilde{V}_{k}(z)+\tilde{G}_{k}(z)^{2}=\tilde{C}_{k}(z)+\tilde{G}_{k}(z)$. For this, it is sufficient to show that the same outer condition holds for $\tilde{C}_{k}(z)$. We prove it by induction on $k$.

Base case for outer condition on $\tilde{V}_{k}(z)+\tilde{G}_{k}(z)^{2}$

The base case, $k=0$, is trivial, since $\tilde{C}_{0}(z)=0$.

Inductive step for outer condition on $\tilde{V}_{k}(z)+\tilde{G}_{k}(z)^{2}$

Now we assume that the claim holds for $k-1$, and we prove it for $k$. A bound for $e^{z} \tilde{C}_{j}(z)$ which is uniform in $j$ holds: in the proof of Lemma 10, we proved that, for all $j \geq 0$ and $z \in \mathbb{C}$,

$$
\left|\tilde{C}_{j}(z)\right| \leq|z|^{2} e^{|z|-\Re(z)}
$$

which immediately implies that

$$
\left|e^{z} \tilde{C}_{j}(z)\right| \leq|z|^{2} e^{|z|} .
$$


Thus, for a given $R$ and $\phi \in(0,1)$, there is some $C>0$ such that, whenever $|z| \leq R$, for any $j \geq 0$,

$$
\left|e^{z} \tilde{C}_{j}(z)\right| \leq C e^{\phi|z|} .
$$

Now we demonstrate that the same bound holds for $|z|>R$. Recall that $\phi$ in the case of $\tilde{G}_{k}(z)$ is given by $\cos (\theta)+\epsilon$, for any small enough fixed positive $\epsilon>0$. We define $\hat{\phi}$ to be slightly smaller:

$$
\hat{\phi}=\cos (\theta)+\epsilon / 2
$$

and we note that the de-Poissonization result for $\tilde{G}_{k}(z)$ implies that there is some $\hat{R}>0$ such that, whenever $z \notin \mathcal{C}(\theta)$ and $|z|>\hat{R}$, for any $j \geq 0$,

$$
\left|e^{z} \tilde{G}_{j}(z)\right| \leq e^{\hat{\phi}|z|}
$$

We will use this fact in the induction step for $C_{k}(z)$ as follows: we adopt the same approach as in the expected value case, this time defining

$$
\begin{aligned}
\gamma_{1}(z) & =e^{-q z} \\
\gamma_{2}(z) & =e^{-p z} \\
t(z) & =\tilde{C}_{k-1}(p z)\left(1-e^{-q z}\right)+\tilde{C}_{k-1}(q z)\left(1-e^{-p z}\right)+2 \tilde{G}_{k-1}(p z) \tilde{G}_{k-1}(q z) .
\end{aligned}
$$

The conditions required of $\gamma_{1}(z)$ and $\gamma_{2}(z)$ were already verified, so we proceed to show that

$$
e^{\Re(z)}|t(z)| \leq \frac{1}{3} e^{\phi|z|}
$$

for $|z|>R$, for some $R>0$ independent of $k$. We again choose $\phi=\cos (\theta)+\epsilon$, and applying the induction hypothesis and inequality (62) gives

$$
\left|e^{z} t(z)\right| \leq C\left[e^{\phi p|z|}\left|e^{q z}-1\right|+e^{\phi q|z|}\left|e^{p z}-1\right|+C_{2} e^{\hat{\phi}|z|}\right] .
$$

The rest of the induction step goes exactly as in the expected value case, so we omit it.

Inner condition on $\tilde{V}_{k}(z)$

As for the inner conditions, both follow from the asymptotic expansions for $\tilde{G}_{k}(z)$ and $\tilde{V}_{k}(z)$ derived by inverting their respective Mellin transforms. Both derivations are readily extended to $z \rightarrow \infty$ inside the cone.

Since all conditions of the theorem are satisfied, the remaining task is to show that $n\left[\tilde{G}_{k}^{\prime}(n)\right]^{2}=$ $o\left(\tilde{V}_{k}(n)\right)$. This we do using the Cauchy integral formula for derivatives, followed by upper bounding of the resulting integral expression (the main task will then be to choose an appropriate radius for the integration contour): for a circle $\mathcal{C}$ of any radius $R$ enclosing $n$,

$$
\tilde{G}_{k}^{\prime}(n)=\frac{1}{2 \pi i} \oint_{\mathcal{C}} \frac{\tilde{G}_{k}(\xi)}{(\xi-n)^{2}} \mathrm{~d} \xi
$$

which implies

$$
\left|\tilde{G}_{k}^{\prime}(n)\right| \leq \frac{2 \pi R}{2 \pi} \frac{\left|\tilde{G}_{k}\left(\xi_{*}\right)\right|}{R^{2}} \leq\left|\tilde{G}_{k}\left(\xi_{*}\right)\right| / R
$$

where $\xi_{*}=\operatorname{argmax}_{x \in \mathcal{C}}\left|\tilde{G}_{k}(x)\right|$. Now, since $\tilde{G}_{k}(n)=O\left(n^{\beta(\alpha)} / \sqrt{\log n}\right)$, and $\xi_{*}$ is not too different from $n$, we expect that $\tilde{G}_{k}\left(\xi_{*}\right)=O\left(n^{\beta(\alpha)} / \sqrt{\log n}\right)$ as well. Provided that we can show this, if we choose

$$
R=n^{\Delta} / \Psi(n)
$$


for some $\Delta>0$ and slowly growing function $\Psi(n)$ which we will determine later, our bound becomes

$$
n \tilde{G}_{k}^{\prime}(n)^{2}=O\left(n^{1+2 \beta(\alpha)-2 \Delta} \Psi(n)^{2} / \log n\right),
$$

and we would like to enforce the conditions

$$
1+2 \beta(\alpha)-2 \Delta \leq \beta(\alpha)
$$

and

$$
\Delta \leq 1
$$

along with

$$
\Psi(n)^{2} / \log n=o(1 / \sqrt{\log n})
$$

and $\Psi(n) \stackrel{n \rightarrow \infty}{\longrightarrow} \infty$ (so that, for any $\Delta, R=o(n)$ ). Choosing $\Psi(n)$ to satisfy these conditions is easy: we simply require that

$$
\Psi(n)^{2}=o(\sqrt{\log n}) \Longrightarrow \Psi(n)=o\left((\log n)^{1 / 4}\right),
$$

so that we can choose, say, $\Psi(n)=\log \log n$. It is easy to see that, for any $\alpha$, there exists some $\Delta$ which satisfies both conditions simultaneously:

$$
1+2 \beta(\alpha)-2 \Delta \leq \beta(\alpha) \Longleftrightarrow \frac{1+\beta(\alpha)}{2} \leq \Delta,
$$

and

$$
\frac{1+\beta(\alpha)}{2} \leq 1 \Longleftrightarrow \beta(\alpha) \leq 1
$$

This last inequality is true for any $\alpha$ within the range under consideration. With these choices,

$$
n \tilde{G}_{k}^{\prime}(n)^{2}=o\left(n^{\beta(\alpha)} / \sqrt{\log n}\right),
$$

so $n \tilde{G}_{k}^{\prime}(n)^{2}=o\left(\tilde{V}_{k}(n)\right)$, provided that we can show that $\tilde{G}_{k}\left(\xi_{*}\right)=O\left(\tilde{G}_{k}(n)\right)$. To do this, the plan is to show that we can apply Theorem 1 to derive asymptotics for $\tilde{G}_{k}\left(\xi_{*}\right)$. First, we verify that $\xi_{*}$ remains within a cone around the positive real axis. Fixing some $\theta \in(0, \pi / 2)$ for the angle made with respect to the positive real axis, let $A$ denote the point of the form $n+i t$, for some $t \in \mathbb{R}^{+}$, which lies on the boundary of the cone. Furthermore, let $B$ denote the point on the boundary of the cone which lies above the real axis and is nearest to $n$. Then we have

$$
|A-n|=t
$$

and

$$
\frac{t}{n}=\tan (\theta) \Longrightarrow t=\tan (\theta) n=\Theta(n) .
$$

Next, we note that the angle made between the line segment connecting $n$ and $B$ and that connecting 0 and $B$ must be $\pi / 2$, and we denote by $\phi$ the angle between the segments connecting 0 and $n$ and $n$ and $B$. We have, easily, $\phi=\pi / 2-\theta$, and it is trivial to see that the angle between the segments $n$ to $B$ and $n$ to $A$ is $\theta$. Thus, we have that the length of the segment connecting $n$ and $B$ (i.e., the radius of a ball centered at $n$ with maximum volume contained in the cone) is given by

$$
\cos (\theta)=\frac{|B-n|}{|A-n|} \Longrightarrow|B-n|=|A-n| \cos (\theta)=\Theta(n) \cos (\theta)=\Theta(n),
$$

so that, since $R=o(n), \xi_{*}$ must be inside the cone. We then examine the relationship between $k$ and $\xi_{*}$. Since $n=\xi_{*}(1+o(1))$,

$$
k \sim \alpha \log n=\alpha \log \left(\xi_{*}(1+o(1))\right)=\alpha \log \xi_{*}+o(1),
$$

so that $k \sim \alpha \log \xi_{*}$. Applying Theorem 1 then shows that $\tilde{G}_{k}\left(\xi_{*}\right)=O\left(\tilde{G}_{k}(n)\right)$.

This completes the proof. 


\subsubsection{De-Poissonization for the central limit theorem}

The final step of the proof is inversion of the Poisson transform to recover a central limit theorem for the Bernoulli model. That is, knowing asymptotic information about $\tilde{Q}_{k}(u, z)$, our goal is to recover $Q_{n, k}(u)$. The Cauchy integral formula gives

$$
Q_{n, k}(u)=\frac{n !}{2 \pi i} \oint_{|z|=n} e^{z} \tilde{Q}_{k}(u, z) z^{-n-1} \mathrm{~d} z
$$

where the integration contour (we denote it by $\mathcal{C}$ ) is the circle centered at 0 with radius $n$. The evaluation of this integral will proceed in two stages. We expect that the main contribution will come from a small arc around the positive real axis, so we fix a cone around the positive real axis, and we show that the contribution outside the cone is negligible (by a lemma which we will soon state). Next, we break the remaining part of the contour into inner tails and a central region. The inner tails we show to be negligible using Lemma 14, the Taylor expansion for the cosine function around 0 , and a careful choice of the split into the inner tails and the central region. Finally, the central region is evaluated using the expansion for $\tilde{Q}_{k}(u, z)$ derived above, as well as the fact that

$$
\frac{1}{\sqrt{2 \pi}} \int_{-\infty}^{\infty} e^{-x^{2}} \mathrm{~d} x=1
$$

Let $\theta$ be an angle in $(0, \pi / 2)$ for which

$$
\tilde{l}_{k}(u, z)=z+\tilde{G}_{k}(z) \frac{\tau}{\sigma_{n, k}}+\tilde{V}_{k}(z) \frac{\tau^{2}}{2 \sigma_{n, k}^{2}}+R\left[\tilde{l}_{k}(u, z) \frac{\tau^{3}}{3 ! \sigma_{n, k}^{3}}+O\left(\sigma_{n, k}^{-1}\right),\right.
$$

with $R\left[\tilde{l}_{k}(u, z)=O\left(n^{\beta(\alpha)}\right)\right.$. This $\theta$ is guaranteed to exist by the analysis in Section 3.3.

We require a final estimate on the growth of $Q_{j}(u, x)$ in order to upper bound the outer tails of $(63)$ :

Lemma 19 (Growth of $Q_{j}(u, x)$ outside a cone). Let $\theta \in(0, \pi / 2)$. Then there is some $\alpha \in(0,1)$ and $x_{0}>0$ such that, provided $x \notin \mathcal{C}(\theta)$ and $|x| \geq x_{0}$,

$$
\left|Q_{j}(u, x)\right| \leq e^{\alpha|x|}
$$

uniformly in $j \leq k$.

Proof. We prove a slightly different claim: that, for each $\theta$ with $|\theta| \in(0, \pi / 2)$, there is some $\alpha<1$ and $x_{0}>0$ such that, for all $j \leq k$, if $x \notin \mathcal{C}(\theta)$ and $|x| \geq x_{0}$, then

$$
\left|Q_{j}(u, x)\right| \leq e^{\alpha|x|-1}
$$

Note the additional term of -1 in the exponent. This we prove by induction in $j$. To accomplish this, for each $j$, we prove that the inequality holds for $|x| \in\left[x_{0}^{\prime}, x_{0}\right)$ with $x_{0}^{\prime}=q x_{0}$, and we then use this and induction on increasing domains to prove that it holds for $|x| \geq x_{0}$.

Base case for $j$ induction

For the base case, recall that $Q_{0}(u, x)=e^{x}-x(1-u)$, so that

$$
\left|Q_{0}(u, x)\right| \leq e^{|x| \cos (\arg (x))}+|x||1-u| .
$$

For appropriately chosen $\alpha$ (say, $\cos (\theta)+\epsilon$, for any small enough positive $\epsilon$ ), $|x|$ can be made large enough so that this satisfies the claimed property. That is, there is some $x_{0}^{\prime}$ for which the stated inequality holds whenever $|x| \geq x_{0}^{\prime}$. We define $x_{0}$ to be $x_{0}^{\prime} / q$. 


\section{Induction on $j$, base case for increasing domains induction}

For the induction on $j$, we assume that the claim holds for $j-1$, and we aim to prove it for $j$. To do this, we use induction on increasing domains. To verify the claim for $|x| \in\left[x_{0}^{\prime}, x_{0}\right)$, we apply Lemma 12, which is justified because $|x|<x_{0}$, to conclude that

$$
Q_{j}(u, x) \sim e^{x},
$$

so that

$$
\left|Q_{j}(u, x)\right| \sim\left|e^{x}\right|=e^{|x| \cos (\theta)},
$$

and, provided $x_{0}$ is sufficiently large,

$$
\left|Q_{j}(u, x)\right| \leq e^{\alpha|x|-1}
$$

which gives us the base case of the increasing domains induction.

\section{Increasing domains inductive step}

We now proceed to the inductive step. Applying the functional equation and the triangle inequality, then the inductive hypotheses,

$$
\left|Q_{j}(u, x)\right| \leq e^{\alpha|x|-2}+4 e^{\alpha p|x|-1}=e^{\alpha|x|-2}\left(1+4 e^{-\alpha q|x|+1}\right)
$$

Next, note that, since $e^{-\alpha q|x|+1}=o(1)$ as $|x| \rightarrow \infty$, the second factor in the above product satisfies

$$
1+4 e^{-\alpha q|x|+1} \sim e^{4 e^{-\alpha q|x|+1}}=e^{o(1)},
$$

so that, provided we choose $|x|$ large enough,

$$
\left|Q_{j}(u, x)\right| \leq e^{\alpha|x|-1}
$$

which completes the proof.

Bounding the outer tails

The outer tails of (63) then become

$$
\begin{aligned}
\frac{n !}{2 \pi i} \int_{|\arg (z)|>\theta} e^{z} \tilde{Q}_{k}(u, z) z^{-n-1} \mathrm{~d} z & \sim \frac{\sqrt{2 \pi n} n^{n} e^{-n}}{2 \pi i} \int_{|\arg (z)|>\theta} e^{z} \tilde{Q}_{k}(u, z) z^{-n-1} \mathrm{~d} z \\
& =\frac{n^{n+1 / 2} e^{-n}}{\sqrt{2 \pi} i} \int_{|\arg (z)|>\theta} e^{z} \tilde{Q}_{k}(u, z) z^{-n-1} \mathrm{~d} z
\end{aligned}
$$

where we used Stirling's formula. Taking absolute values and applying Lemma 19 gives an upper bound of

$$
\frac{n^{n+1 / 2} e^{-n+\alpha n}}{\sqrt{2 \pi}} 2 \pi n n^{-n-1}=n^{O(1)} e^{-n(1-\alpha)},
$$

which is exponentially decaying in $n$, since $\alpha<1$.

\section{Bounding the inner tails}

Now we bound the inner tails. Specifically, we let $\psi=n^{-\delta}$, for some $\delta>0$ to be determined, and the inner tails consist of that part of the contour where $|\arg (z)| \in(\psi, \theta]$. The choice of $\psi$ is dictated by two opposing forces: it must be large enough that the inner tails are negligible but 
small enough so that the central part is easy to estimate precisely. In the range of integration of the inner tails, we have the estimate

$$
\left|e^{z} \tilde{Q}_{k}(u, z)\right|=e^{n \cos (\arg (z))(1+o(1))} \leq e^{n \cos (\psi)},
$$

by Lemma 14. Taylor expanding $\cos (\psi)$ around 0 gives

$$
\exp \left(n\left(1-\frac{\psi^{2}}{2 !}+O\left(\psi^{4}\right)\right)\right)
$$

We will require that $n \psi^{2}=n^{1-2 \delta} \stackrel{n \rightarrow \infty}{\longrightarrow} \infty$, which translates to

$$
1-2 \delta>0 \Longrightarrow \delta<1 / 2 \text {. }
$$

Then we can upper bound the inner tails by

$$
\frac{n^{n+1 / 2} e^{-n}}{\sqrt{2 \pi}} 2 \pi n\left|e^{z} \tilde{Q}_{k}(u, z)\right| n^{-n-1} \leq n^{O(1)} e^{-n+n\left(1-\frac{\psi^{2}}{2 !}+O\left(\psi^{4}\right)\right)}=n^{O(1)} e^{-n^{1-2 \delta}(1+o(1))},
$$

which is exponentially decaying in $n$, so negligible.

\section{Estimating the central region}

Now we estimate the central part. Inside the integral, letting $\phi$ denote $\arg (z)$, we can expand $e^{z} z^{-n-1}$ as

$$
e^{z} z^{-n-1}=e^{n e^{i \phi}-(n+1) \log \left(n e^{i \phi}\right)}=e^{n\left(1+i \phi-\frac{\phi^{2}}{2}+O\left(\phi^{3}\right)\right)-(n+1) \log n-i \phi}=e^{n} n^{-n-1} e^{-\frac{n \phi^{2}}{2}(1+o(1))} .
$$

Multiplying by the $e^{-n} n^{n+1 / 2}$ outside the integral gives

$$
\frac{1}{\sqrt{n}} e^{-\frac{n \phi^{2}}{2}(1+o(1))}
$$

Applying the analysis of $\tilde{Q}_{k}(u, z)$,

$$
\begin{aligned}
\tilde{Q}_{k}(u, z) & =\exp \left(\tilde{G}_{k}(z) \frac{\tau}{\sigma_{n, k}}+\frac{\tau^{2}}{2}+O\left(\sigma_{n, k}^{-1}\right)\right) \\
& =\exp \left(\tilde{G}_{k}(n) \frac{\tau}{\sigma_{n, k}}+\frac{\tau^{2}}{2}+O\left(\tilde{G}_{k}^{\prime}(n)(z-n)\right) \frac{\tau}{\sigma_{n, k}}+O\left(\sigma_{n, k}^{-1}\right)\right),
\end{aligned}
$$

where we note that $\tilde{G}_{k}(n)=\Theta\left(n^{\beta(\alpha)} / \sqrt{\log (n)}\right)$, while $\tilde{G}_{k}^{\prime}(n)=\tilde{O}\left(n^{\beta(\alpha)-1}\right)$. Since $\beta(\alpha)-1 \leq 0$ and $z-n=O(\psi)=n^{-\Omega(1)}$, the third term is $o\left(\sigma_{n, k}^{-1}\right)$. That is,

$$
\tilde{Q}_{k}(u, z)=\exp \left(\tilde{G}_{k}(n) \frac{\tau}{\sigma_{n, k}}+\frac{\tau^{2}}{2}+O\left(\sigma_{n, k}^{-1}\right)\right) .
$$

Putting these estimates together, we see that the contribution of the central region is given by

$$
\frac{\exp \left(\tilde{G}_{k}(n) \frac{\tau}{\sigma_{n, k}}+\frac{\tau^{2}}{2}+O\left(\sigma_{n, k}^{-1}\right)\right)}{\sqrt{2 \pi n}} \int_{-\psi}^{\psi} e^{-\frac{n \phi^{2}}{2}(1+o(1))} \mathrm{d} \phi \text {. }
$$

It is easy to see that we can complete the tails, and then we make the substitution $x=n^{-1 / 2} \phi$, which gives

$$
\frac{\exp \left(\tilde{G}_{k}(n) \frac{\tau}{\sigma_{n, k}}+\frac{\tau^{2}}{2}+O\left(\sigma_{n, k}^{-1}\right)\right)}{\sqrt{2 \pi}} \int_{-\infty}^{\infty} e^{-x^{2}} \mathrm{~d} x
$$


Since the integral, along with the factor $\frac{1}{\sqrt{2 \pi}}$, becomes 1 , we have, finally,

$$
\mathbb{E}\left[e^{B_{n, k} \frac{\tau}{\sigma_{n, k}}}\right]=\exp \left(\tilde{G}_{k}(n) \frac{\tau}{\sigma_{n, k}}+\frac{\tau^{2}}{2}+O\left(\sigma_{n, k}^{-1}\right)\right)
$$

and applying the Lévy continuity theorem shows that the claimed central limit theorem holds for properly normalized $B_{n, k}$.

\section{References}

[1] Milton Abramowitz and Irene A. Stegun. Handbook of Mathematical Functions with Formulas, Graphs, and Mathematical Tables, volume 55 of National Bureau of Standards Applied Mathematics Series. For sale by the Superintendent of Documents, U.S. Government Printing Office, Washington, D.C., 1964.

[2] Luc Devroye. A note on the probabilistic analysis of patricia trees. Random Struct. Algorithms, 3(2):203-214, March 1992.

[3] Luc Devroye. Laws of large numbers and tail inequalities for random tries and patricia trees. Journal of Computational and Applied Mathematics, 142:27-37, 2002.

[4] Luc Devroye. Universal asymptotics for random tries and patricia trees. Algorithmica, 42(1):11-29, 2005.

[5] Michael Drmota and Wojciech Szpankowski. The expected profile of digital search trees. J. Comb. Theory Ser. A, 118(7):1939-1965, October 2011.

[6] Philippe Flajolet. The ubiquitous digital tree. STACS, 3884:1-22, 2006.

[7] Philippe Flajolet, Xavier Gourdon, and Philippe Dumas. Mellin transforms and asymptotics: Harmonic sums. Theoretical Computer Science, 144:3-58, 1995.

[8] Philippe Flajolet and Robert Sedgewick. Analytic Combinatorics. Cambridge University Press, Cambridge, UK, 2009.

[9] Philippe Jacquet, Charles Knessl, and Wojciech Szpankowski. A note on a problem posed by D. E. Knuth on a satisfiability recurrence. Combinatorics, Probability, and Computing, 2014.

[10] Philippe Jacquet and Wojciech Szpankowski. Analytical depoissonization and its applications. Theor. Comput. Sci., 201(1-2):1-62, July 1998.

[11] Svante Janson and Wojciech Szpankowski. Analysis of an asymmetric leader election algorithm. Electronic J. Combin, 4:1-6, 1996.

[12] Ramin Kazemi and Mohammad Vahidi-Asl. The variance of the profile in digital search trees. Discrete Mathematics and Theoretical Computer Science, 13(3):21-38, 2011.

[13] Donald E. Knuth. The Art of Computer Programming, Volume 3: (2nd ed.) Sorting and Searching. Addison Wesley Longman Publishing Co., Inc., Redwood City, CA, USA, 1998.

[14] Abram Magner. Profiles of PATRICIA Tries. PhD thesis, Purdue University, December 2015. 
[15] Abram Magner, Charles Knessl, and Wojciech Szpankowski. Expected external profile of patricia tries. Proceedings of the Eleventh Workshop on Analytic Algorithmics and Combinatorics, pages 16-24, 2014.

[16] Moni Naor and Udi Wieder. Novel architectures for P2P applications: the continuous-discrete approach. In Proceedings of the fifteenth annual ACM symposium on Parallel algorithms and architectures, SPAA '03, pages 50-59, New York, NY, USA, 2003. ACM.

[17] G. Park, H. Hwang, P. Nicodème, and W. Szpankowski. Profiles of tries. SIAM Journal on Computing, 38(5):1821-1880, 2009.

[18] B. Pittel. Asymptotic growth of a class of random trees. Ann. Probab., 18:414-427, 1985.

[19] Boris Pittel and Herman Rubin. How many random questions are needed to identify $n$ distinct objects? Journal of Combinatorial Theory, Series A, 55(2):292-312, 1990.

[20] M. Regnier and P. Jacquet. New results on the size of tries. Information Theory, IEEE Transactions on, 35(1):203-205, 1989.

[21] Wojciech Szpankowski. Patricia tries again revisited. J. ACM, 37(4):691-711, October 1990.

[22] Wojciech Szpankowski. Average Case Analysis of Algorithms on Sequences. John Wiley \& Sons, Inc., New York, NY, USA, 2001.

[23] Edward Charles Titchmarsh. The Theory of Functions. Oxford University Press, 1939. 